\title{
NONLINEAR AND INERTANT ACOUSTIC METAMATERIALS AND THEIR \\ DEVICE IMPLICATIONS
}

\author{
By \\ PRATEEK P. KULKARNI \\ Bachelor of Engineering in Mechanical Engineering \\ Birla Institute of Technology \\ Mesra, India \\ 2012
}

Submitted to the Faculty of the

Graduate College of the

Oklahoma State University

in partial fulfillment of

the requirements for

the Degree of

MASTER OF SCIENCE

December, 2016 


\section{NONLINEAR AND INERTANT ACOUSTIC METAMATERIALS AND THEIR DEVICE IMPLICATIONS}

Thesis Approved:

Dr. James M. Manimala

\begin{tabular}{c} 
Thesis Adviser \\
Dr. James K. Good \\
\hline Dr. Xiaoliang Jin \\
\hline
\end{tabular}




\title{
ACKNOWLEDGEMENTS
}

\author{
To failures that teach \\ To failures, that inspire to succeed \\ To failures that go unnoticed, that go undocumented \\ To everyone, that failed to help, \\ To everyone that I failed to ask for help
}

I thank the child me for having stayed with me, as the clock of my life ticked

I thank the initial and boundary conditions my life was subjected to, for having made me stand here.

I thank every parameter, every person that has been involved in some way for being involved in that very way.

I would like to acknowledge the serene, truly unconditional support from my parents who had not the remotest clue of what I was doing and yet smiled in appreciation. I wish to never let any of my work go without mention of their struggles.

I thank my sister, Pratima Kulkarni for being there.

For truly being there all of my life. For being the harsh teacher, and the tender mother at times. I could never imagine going past class 10 without her support. She in truth has given birth and nurtured the engineer in me. She in truth is the godmother of most machines I have built, or, will(definitely) build.

This thesis, in particular, has to be a product of a pair of meticulously guiding eyes; that of my adviser, Dr. James M. Manimala, who has been a source of inspiration and moral support; whose patience exceeds mine; while I thought mine was the limit.

I will perhaps be indebted to his presence in my life, for he's been more than a graduate adviser perhaps without his own knowledge - a teacher.

I would like to express my gratitude to Dr. James K. Good for the insights he provided during and after the 'Finite Element Methods' course, which helped me throughout the research endeavors. I would also like to thank Dr. Xiaoliang Jin for supporting me with manufacturing of the test-article.

I would like to thank Zeel Maheshwari, Arjun Dhamodharan, Barrett Lee, Ryan Aiken, Matthew Liao, Vishnu Paidimarri, Aditya Aggarwal, Roy for their support.

I wish the cosmos truly transmits my gratitude to every person involved.

iii

Acknowledgements reflect the views of the author and are not endorsed by committee members or Oklahoma State University. 
Name: PRATEEK P. KULKARNI

Date of Degree: DECEMBER, 2016

Title of Study: NONLINEAR AND INERTANT ACOUSTIC METAMATERIALS AND THEIR DEVICE IMPLICATIONS

Major Field: MECHANICAL AND AEROSPACE ENGINEERING

Abstract:

Acoustic Metamaterials (AM) are a class of artificial structural materials that derive their unique dynamic properties, not just from material constituents but more so from engineered local configurations. Tailoring these local configurations have been shown to impart unusual mechanical wave manipulation capabilities to AM with potentially novel applications in protective structures; acoustic devices for sensing, noise control, and energy harvesting; and MEMS devices. Most AM require the presence of periodic features that locally exhibit dynamic phenomena like resonance or instability within a host material or structure. A key advantage of the AM design approach is the latitude to explore new local configurations to further enrich their dynamic behavior. The present study focusses on nonlinear and inertant AM configurations and their device implications. Using the method of multiple scales applied to a lumpedparameter effective-mass model, approximate analytical solutions were derived for the amplitude-dependent dispersion curve shifts in nonlinear AM owing to the presence of cubically hardening or softening nonlinearities in local oscillators. Discrete element simulations predict the possibility of realizing passive acoustic control devices such as selective filters, amplitude band-pass filters and directionbiased waveguides using nonlinear AM. A numerical routine to generate root profile geometries that enable contact-based hardening response in tip-loaded cantilever beam resonators was developed and implemented. Experiments on a structural waveguide test article verify the existence and extent of bandgaps and also provide an indication of the passive direction-bias phenomenon. Whereas, incorporating inerters, which are mechanical elements that display a force proportional to the relative acceleration across them, could create structural devices that display frequencydependent negative and even extreme effective-mass and stiffness regimes. Such devices have implications for passive high-pass filters with ultra-low frequency bandgaps that encompass the long wavelength limit and can be realized without the use of any mechanical grounding elements and even for structural networks that can act as a nearly complete mechanical wave inhibitor. Further research on the interactive synergies between nonlinear and inertant configurations and practical strategies to scale and fabricate them could have promise for realizing a new class of AM with enriched dynamics beyond those found in predominantly locally resonant variants that are currently being developed. 


\section{TABLE OF CONTENTS}

Chapter Page

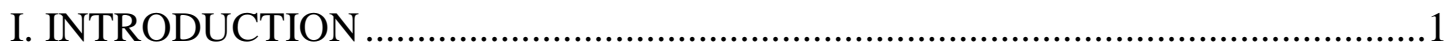

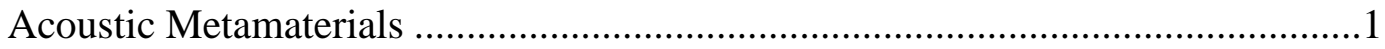

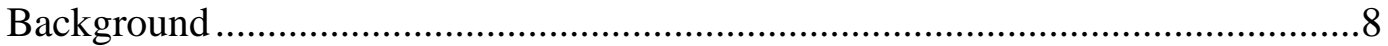

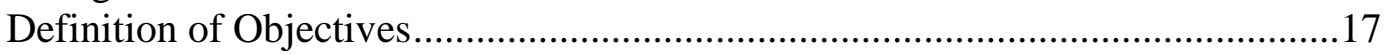

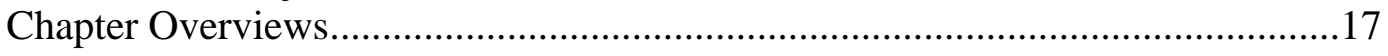

II. NONLINEAR ACOUSTIC METAMATERIALS ..............................................19

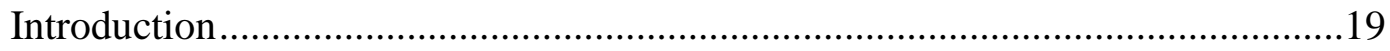

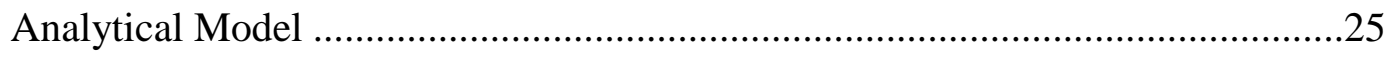

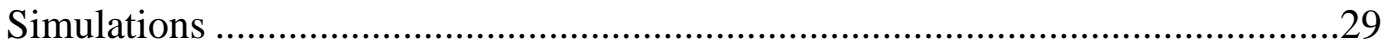

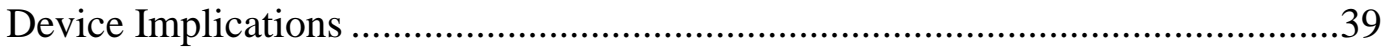

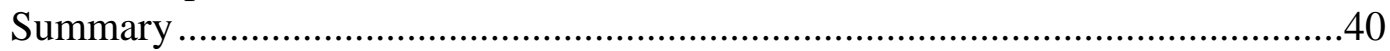

III. EXPERIMENTAL VERIFICATION .......................................................41

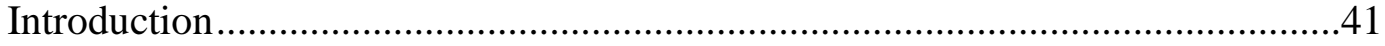

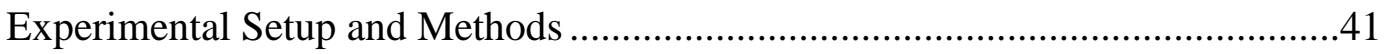

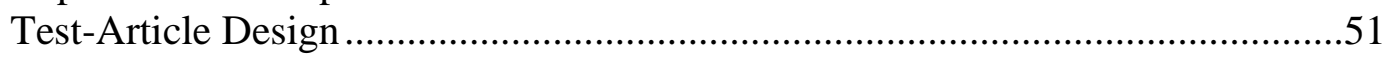

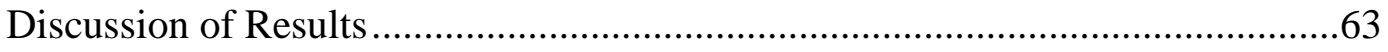

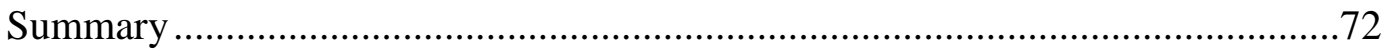

IV. INERTANT ACOUSTIC METAMATERIALS ….......................................... 73

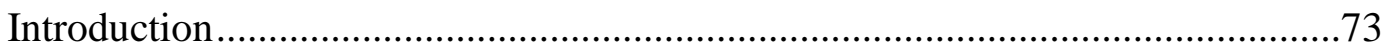

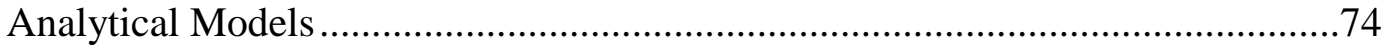

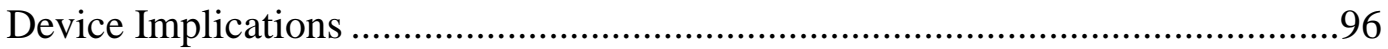

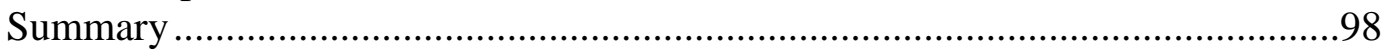


V. CONCLUSIONS AND RECOMMENDATIONS .

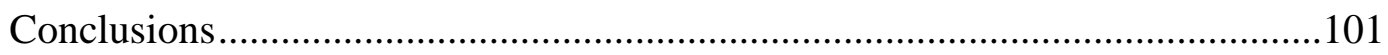

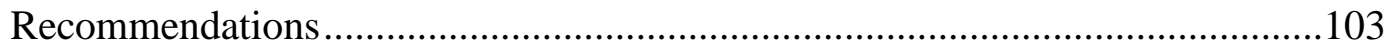

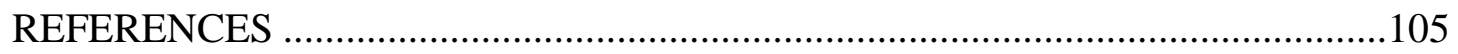

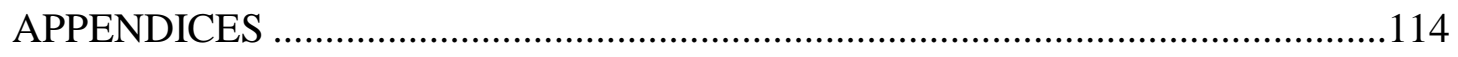




\section{LIST OF TABLES}

Table

Page

Table 2. 1: Propagation characteristics of the proposed direction-biased waveguide

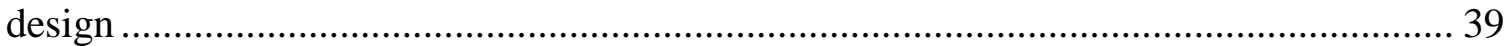

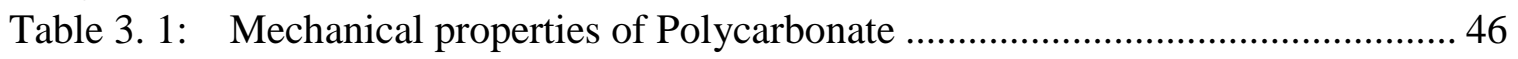

Table 4. 1: Key propagation characteristics of various inertant configurations........... 98 


\section{LIST OF FIGURES}

Figure Page

Figure 1. 1: Examples of metamaterials using (a) Locally resonant lead spheres [11] and (b) Nonlinearity in granular chain [67] ....................................................... 7

Figure 1. 2: One approach to obtain negative stiffness from bistable beam [38].......... 9

Figure 1. 3: Missing parameter relating the relative acceleration across the element. .. 13

Figure 1. 4: Completed force-current analogy by including the inerter [48] ............... 14

Figure 1. 5: Schematic representing a method to realize an inerter using rack and pinion mechanism [48] ........................................................................................ 14

Figure 1. 6: An inerter device manufactured based on ball-screw type design [48]. ... 15

Figure 2. 1: Schematic diagram of the cubic nonlinear duffing oscillator used for simulation in Abaqus. .......................................................................................... 22

Figure 2. 2: Constant amplitude harmonic forcing with incrementally varying

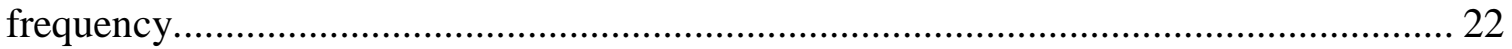

Figure 2. 3: Displacement time history of mass with decreasing frequency forcing.... 23

Figure 2. 4: Displacement time history of mass with increasing frequency forcing. .... 23

Figure 2. 5: Displacement plotted as a function of amplitude in the case of increasing and decreasing forcing frequency. The analytical response of the system with linear

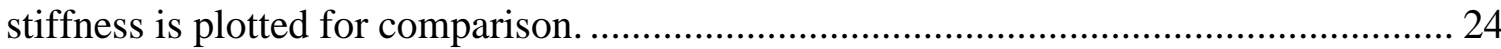

Figure 2. 6: Discrete mass-spring lattice model for an AM with local nonlinear

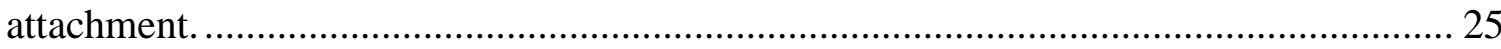

Figure 2. 7: Dispersion curves obtained by perturbation method for LRAM type lattice with softening and hardening type nonlinearity in the local attachment. The excitation frequency normalized with local resonance frequency is plotted against the wave number.

28

Figure 2. 8: Simulation model set up in Abaqus for LRAM. ..................................... 29

Figure 2. 9: Demonstration of bandgap effect through simulations in an LRAM........ 31

Figure 2. 10: Simulation model set up in Abaqus for LRAM. .................................. 31

Figure 2. 11: Demonstration of bandgap effect through simulations for NLAM......... 33

Figure 2. 12: Amplitude-dependent propagation due to nonlinearity.......................... 33

Figure 2. 13: Shift in the frequency of transmitted wave by the NLAM for an incident

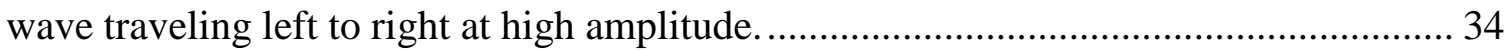

Figure 2. 14: (a) Schematic of the simulation model in Abaqus and (b) mass-spring

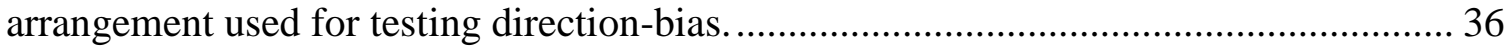

Figure 2. 15: Direction-bias effect obtained in the designed DBWG from simulations.38 
Figure 3. 1: Schematic of the experimental setup.

Figure 3. 2: Assembled experimental set up showing various components of the system.

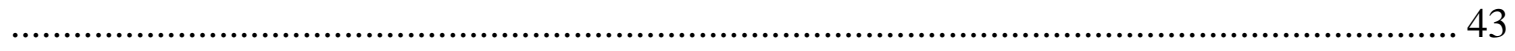

Figure 3. 3: LabVIEW interface developed for signal extraction................................. 44

Figure 3. 4: CAD model and assembled fixture for transmission of force from shaker to

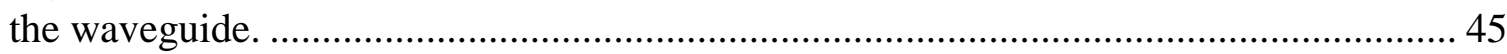

Figure 3. 5: Measurement of wave velocity for the waveguide assembly.................... 47

Figure 3. 6: Snapshot of displacement history of points separated along the length of

waveguide for a $1 \mathrm{kHz}$ continuous sine excitation. ........................................................ 48

Figure 3. 7: Characterizing the behavior of employed boundary condition by comparing the acceleration response at a point on the waveguide.................................................... 49

Figure 3. 8: Computing the host stiffness for an LRAM component. ........................... 51

Figure 3. 9: Technical drawing for the LRAM test-article, showing the detailed

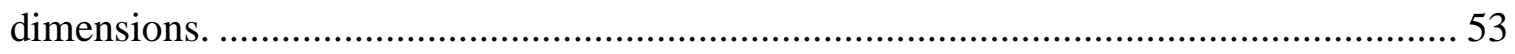

Figure 3. 10: Manufactured LRAM test-article fabricated in a polycarbonate plate..... 53

Figure 3. 11: Experimentally recorded local resonance frequencies of LRAM............ 54

Figure 3. 12: (a) Nonlinear hardening stiffness obtained through contact with root profile. (b) Method of locating coordinates of the support points using beam deflection

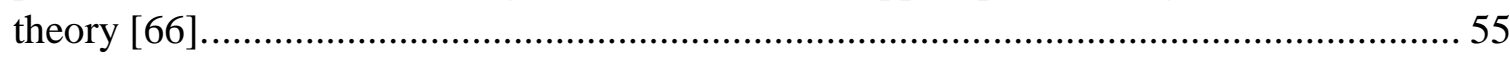

Figure 3. 13: (a) Input stiffness curve with Linear and NLH force-displacement data and (b) Computed cantilever root profile for obtaining the predefined load-displacement

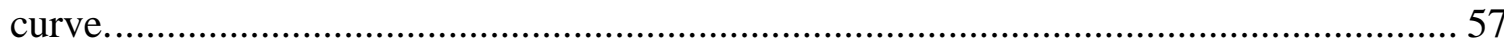

Figure 3. 14: Flow chart to obtain root profile for pre-defined nonlinearity................ 57

Figure 3. 15: Cantilever beam modeled in Abaqus with root profile obtained from

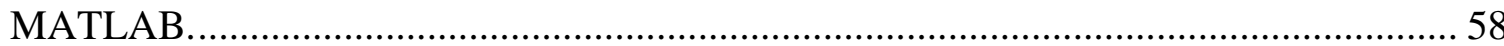

Figure 3. 16: Comparison of designed stiffness against simulation results obtained using Abaqus.......................................................................................................... 59

Figure 3. 17: Technical drawings to achieve linear part of the NLAM test-article (a) Polycarbonate plate with cantilever features and (b) steel tip mass. ................................ 60

Figure 3. 18: First mode shape of designed NLAM unit cell from SolidWorks. ........... 61

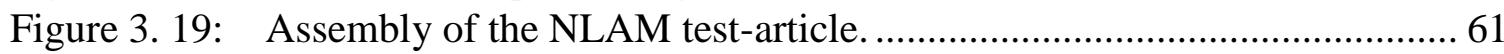

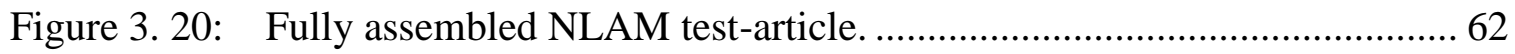

Figure 3. 21: Experimentally recorded local resonance frequencies of NLAM............ 62

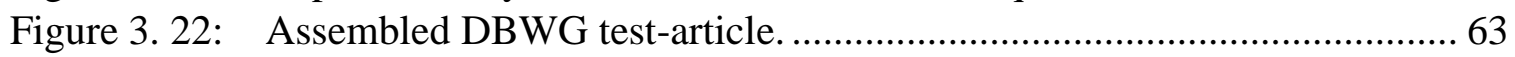

Figure 3. 23: Comparison of experimental and theoretical transmissibility curves for the

LRAM test-article demonstrating the bandgap phenomenon. ............................................ 64

Figure 3. 24: Time history and frequency spectrum of forcing frequencies within and outside theoretical bandgap. The excitations are (a) at $1000 \mathrm{~Hz}$ which is below the lower bound, (b) at $1100 \mathrm{~Hz}$ which is within the bandgap, (c) at $1250 \mathrm{~Hz}$ also within bandgap and (d) $1500 \mathrm{~Hz}$ which is above the upper bound of the bandgap.................................. 66 Figure 3. 25: Comparison of experimental and analytical transmissibility curves for the NLAM test-article demonstrating bandgap phenomenon in linear regime. 67 
Figure 3. 26: Transmissibility curves for the final test-article (NLAM and LRAM joined) for the four cases covering both directions and amplitudes of excitation. .......... 68 Figure 3. 27: Response of the test-article at $1080 \mathrm{~Hz}$, at different conditions - Low amplitude (a) Left to Right, (b) Right to Left and High amplitude (c) Left to Right, (d) Right to Left direction of traverse .................................................................... 71

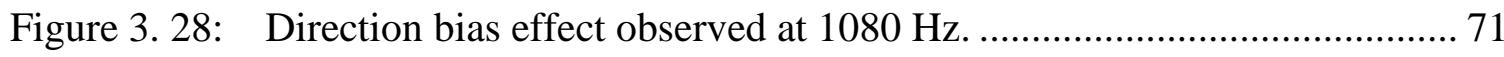

Figure 4. 1: (a) Discrete mass-spring lattice model for a locally resonant acoustic metamaterial and (b) its effective-mass model................................................... 75

Figure 4. 2: (a) Normalized effective-mass, and (b) real and (c) imaginary parts of the

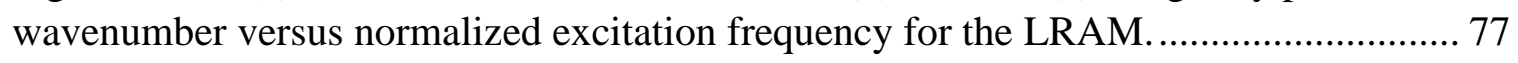

Figure 4. 3: Acoustic metamaterial with purely inertant local attachments. ................ 78 Figure 4. 4: (a) Normalized effective-mass versus normalized inertance and (b) real and (c) imaginary parts of wavenumber versus normalized excitation frequency................. 80 Figure 4. 5: Acoustic metamaterial with local attachments having inerter in parallel with the spring...

Figure 4. 6: (a) Normalized effective-mass, (b) real and (c) imaginary parts of the wavenumber versus normalized excitation frequency and (d) mass ratio vs bandgap limits for various values of inertance for acoustic metamaterial with parallel inertant attachment. 82

Figure 4. 7: Acoustic metamaterial with local attachments having inerter in series with the spring. 83

Figure 4. 8: (a) Normalized effective-mass, (b) real and (c) imaginary parts of the wavenumber versus normalized excitation frequency and (d) mass ratio vs bandgap limits for various values of inertance for acoustic metamaterial with series inertant attachment.

Figure 4. 9: Acoustic metamaterial with lattice structure having inerter in parallel with stiffness. 85

Figure 4. 10: (a) Parallel inertant SDOF system and (b) its equivalent effective-stiffness model. 86

Figure 4. 11: (a) Normalized effective-stiffness and (b) effective-mass normalized excitation frequency for different values of inertance for the parallel inertant lattice configuration. 88

Figure 4. 12: Normalized effective lattice parameter versus normalized excitation frequency for (a) $\mathrm{J} / \mathrm{m}_{1}=1$ and (b) $\mathrm{J} / \mathrm{m}_{1}=100$ along with (c) real and (d) imaginary parts of the wavenumber versus normalized excitation frequency for these cases for the parallel inertant lattice configuration.

Figure 4. 13: Normalized effective lattice parameter versus normalized excitation frequency for (a) $\mathrm{J} / \mathrm{m}_{1}=9$ and (b) $\mathrm{J} / \mathrm{m}_{1}=4.74$ along with (c) real and (d) imaginary parts of the wavenumber versus normalized excitation frequency for these cases for the parallel inertant lattice configuration.

Figure 4. 14: Acoustic metamaterial with lattice structure having inerter in series with stiffness. 
Figure 4. 15: (a) Normalized effective-stiffness and (b) effective-mass versus normalized excitation frequency for different values of inertance for the series inertant lattice configuration.

Figure 4. 16: Normalized effective lattice parameter versus normalized excitation frequency for (a) $\mathrm{J} / \mathrm{m}_{1}=1$ and (b) $\mathrm{J} / \mathrm{m}_{1}=20$ along with (c) real and (d) imaginary parts of the wavenumber versus normalized excitation frequency for these cases for the series inertant lattice configuration. ........................................................................... 94 Figure 4. 17: Normalized effective lattice parameter versus normalized excitation frequency for (a) $\mathrm{J} / \mathrm{m}_{1}=9$ and (b) $\mathrm{J} / \mathrm{m}_{1}=4.74$ along with (c) real and (d) imaginary parts of the wavenumber versus normalized excitation frequency for these cases for the series inertant lattice configuration.

Figure 4. 18: Attenuation factors for the longitudinal wave inhibitor with combined parallel and series inertant lattice configurations having (a) $\mathrm{J} / \mathrm{m}_{1}=4.74$ for PL and $\mathrm{J} / \mathrm{m}_{1}=9$ for SL and (b) $\mathrm{J} / \mathrm{m}_{1}=4.74$ for both PL and SL. 


\section{LIST OF SYMBOLS AND ABBREVIATIONS}

\begin{tabular}{|c|c|}
\hline $\mathrm{AM}$ & Acoustic Metamaterial \\
\hline $\mathrm{D}^{*}$ & Transmissibility \\
\hline DBWG & Direction-Bias Wave Guide \\
\hline$\varepsilon$ & Perturbation Parameter \\
\hline $\mathrm{f}^{*}$ & Amplitude in Frequency Domain \\
\hline $\mathrm{J}$ & Inertance \\
\hline $\mathrm{k}$ & Stiffness \\
\hline $\mathrm{k}_{\mathrm{n}}$ & Nonlinear Stiffness Parameter \\
\hline LB & Lower Bound \\
\hline LRAM & Locally Resonant Acoustic Metamaterial \\
\hline NLAM & Nonlinear Acoustic Metamaterial \\
\hline NLH & Nonlinear Hardening (Stiffness) \\
\hline NLS & Nonlinear Softening (Stiffness) \\
\hline PI & Parallel Inertant \\
\hline PL & Parallel Inertant Lattice \\
\hline SI & Series Inertant \\
\hline SL & Series Inertant Lattice \\
\hline $\mathrm{u}$ & Displacement \\
\hline UB & Upper Bound \\
\hline WI & Wholly Inertant \\
\hline
\end{tabular}


$\Omega$

$\Gamma$
Normalized Frequency

Nonlinearity Parameter 


\section{CHAPTER I}

\section{INTRODUCTION}

\subsection{Acoustic Metamaterials}

Starting with the invention of the wheel, mankind has moved significantly forward with engineering, considering the successful exploration of celestial bodies once regarded as gods. From such humble origins as stones and wood, the human race has grown to an age of self-made materials such as plastics. With the proliferation of human life form, there has been an explosion in the population of machinery and equipment as well.

The ever-increasing need for improved materials has brought science to a point of engineering materials per requirement by a combination of different materials - composites. With advancement in the modern material fabrication process, research on such composite materials witnessed a steep rise [1]. We may have made another incremental development to the previous statement, however - by designing materials not merely by combination but by strategically modifying the defining microstructure itself. This structural modification gives the material the ability to display particular desirable dynamic behavior that is otherwise not a property of naturally occurring materials. Such materials which draw their characteristic properties from their microstructure are called 'Metamaterials'. Metamaterials exhibit unusual properties that are otherwise not present in naturally occurring materials such as negative refractive index. The term was used [2] to describe a three-dimensional periodic cellular configuration, not readily available in nature. 
Despite their superior properties over natural materials, composites suffer from the limitation of their properties being derived from constituent materials; i.e. their overall behavior is a function of constituent materials.

Systems such as machinery, automobile, space vehicles and such, are in constant interaction with dynamic forces inherent in their environment of the application. Terrain topography, wind loading, ocean currents are a few examples of sources that induce dynamic load. This results in a high demand placed on effects that adversely affect the normal operation of machinery. Vibration is one such example. The constant interaction between system and environment; or even the internal working mechanisms itself may result in undesirable vibrations induced in the system which in some cases may jeopardize normal functioning. Such effects can be suppressed by careful design and integration of vibration isolators within the system, which by virtue of their natural frequency act as an energy sink for vibration in the close neighborhood of that frequency.

Another approach is to employ energy harvesting devices in cases wherein, such dissipation of energy via stray vibrations is productively utilized by using the harmonic forcing as a means to sequester energy by employing tuned mass resonators.

Further, the traditional limitation imposed on material design is the inverse relation between compliance and stiffness. The ability to produce stiff materials with high material dissipation capacity has driven the science community to push the range of investigations further, to design efficient structures with vibration and shock absorption properties without loss of static stiffness. In order to do so, research has inevitably turned toward engineering materials at the structural level, more so toward the defining microstructure level imparting unique properties to the resulting 'metamaterial'. This study deals with the case of 'Acoustic Metamaterials (AM)' wherein, the enriched dynamic properties are studied intensively. 
With an increased interest in material design and development, investigation of various configurations to address specific needs and applications in engineering is an indispensable part of science today.

Studies of metamaterials are a relatively recent development; however, the concepts arose quite long back in 1888. The ability to achieve zero velocity for longitudinal light waves were suggested by William Thomson to be possible, given that ether could have negative compressibility [3]. It should be noted that Ether was considered as an elastic medium in those times. Metamaterials have been widely accepted in the field of Electromagnetic (EM) materials, followed by acoustic materials. In the year 1968, Veselago [4] first postulated the possibility of materials bearing negative magnetic permeability $(\mu)$ and electric permittivity $(\varepsilon)$, causing the refractive index to become negative, which is not a characteristic of naturally occurring materials. The concept of metamaterials did not gain much acclaim until after Pendry [5] proposed the possibility of making left-handed metamaterials theoretically; after which, there was a surge of interest in this field and the investigations to obtain more unorthodox behavior from materials continues. This concept of negative properties was a preposterous area of research until the study on EM metamaterials concluded such investigations as feasible [6] by providing experimental evidence of negative refractive index. The capability of manipulating the intrinsic electromagnetic properties mentioned $(\mu, \varepsilon)$ leads to a theory called 'transformation optics' [7], allowing an accurate control of wave propagation characteristics of waveguides. The technique was used in cloaking of objects from EM radiation [8].

The mathematical analogy of acoustic and electromagnetic waves alluded to the possibility of such novel properties being realized for applications in acoustic devices. The respective negative parameters in the acoustic domain would translate to mass density and modulus. The experimental evidence of negative refractive index further motivated investigation to find materials with negative mass density and modulus. Studies for the pursuit of attainment of such 
materials resulted in the investigation of structures containing ordered discontinuities arising from the addition of a resonant substructure to the host material. A classic example and the most prominently explored of this case is the Locally Resonant Acoustic Metamaterial (LRAM) whose nomenclature is self-explanatory - these materials have a resonant mass inclusion to the host structure. Martinez et al [9] reported one of the first frequency dependent attenuation of sound in a periodic structure with a periodicity ranging from a few centimeters to a meter, where the testarticle was a sculpture by Eusebio Sempere exhibited at Juan March Foundation in Madrid. Though theoretical, the unusual dynamic behavior of structures with resonators was known since long, as one of the earliest demonstrations of the gap effect for acoustic waves was given by Vincent [10] in 1898 using nonlinear oscillators. The sculpture (test-article) employed by Martinez et al [9] consisted of hollow stainless steel cylinders distributed periodically in simple cubic symmetry. The cylinders were fixed on a circular platform with a $4 \mathrm{~m}$ diameter. The transmission characteristics were found to be a function of the frequency of sound, throwing light on the phenomenon of frequency dependent attenuation in bandgaps in periodic structures. This study was followed by another experimental investigation of acoustic transmission in a twodimensional periodic array consisting of rigid cylinders in two geometrical arrangements - square and triangular. Again, a dip in sound transmission (defined by filling fraction in this case) was observed depending on the frequency of sound owing to the periodicity and nature of geometry under investigation. Liu et al [11] demonstrated experimentally the existence of frequency dependent band gaps in a test-article containing sonic crystals of known resonant frequency (400 Hz). This test-article consisted of a metal mass inclusion in an epoxy host matrix using a silicone rubber layer. The inner core acts as a mass attached by a stiffness provided by the rubber layer. Sonic transmission measurements from microphones revealed dips in transmission at $400 \mathrm{~Hz}$ and $1100 \mathrm{~Hz}$. Further, still, the authors make a reference to the resonance induced negative stiffness and corroborate the conviction held about possible negative mechanical properties in materials. Sun et al [12] state that the negative effective mass is an effect of the modeling method employed 
to capture the physics of LRAM. In their study [12] they demonstrate that it is due to inaccurate modeling that negative effective mass comes into the picture. By using a different modeling approach - treating an infinite 'mass-in-mass' lattice as a continuum, the effective mass is shown to be not necessarily negative for the effect of bandgap to be retained. They further model the same lattice using a microstructure continuum model and again demonstrate a bandgap with nonnegative effective density. Nevertheless, negative effective parameters have been reported in many instances both as theoretical investigations and experimental records. The physical mechanism that gives rise to the apparent negative mass is based on the phase associated with the assumed harmonic motion of a mass - essentially the inclusion mass moving out of phase with respect to the external/host structure.

Many types of research adopted the model [11] described by Liu et al [13-15], where sonic crystals were fabricated along the lines of the concept of locally resonant metamaterials. Lead spheres (shown in Figure 1.1) coated with compliant material acted locally resonant masses, which resulted in this material to decay sound waves in the $0.2-2 \mathrm{kHz}$ range. Experimental investigation of frequency dependent propagation along a beam on a larger scale include the study by Xiao et al [16] wherein beam like resonators are pivoted on a host beam and measurements taken with the accelerometer on the two ends of the host beam. The spatial decay of waves was reported to be in good agreement with theoretical prediction and numerical analysis of the system. Smith et al [17] carried out a study that included a similar set up with resonant features. This was attained with the help of nuts and washers mounted on an Aluminum beam via a layer of rubber. This system however employed a non-reflective boundary by embedding the far end of the beam in the sand (near end subject to harmonic excitations).

At this point, the frequency-dependent effective-density [11] and modulus [18] which could become negative gave rise to adjustable bandgaps $[12,18]$ and propagation modes with negative group velocity [19]. Several other studies on LRAM were carried out, employing different 
mechanisms to achieve local resonance. Helmholtz resonators were employed to obtain ultrasonic metamaterials [20]; elastic membranes arranged as one-dimensional arrays [21, 22]; sonic crystals as previously detailed [11] locally resonant features within a host material [23, 24]. Further studies including damping in the structures included the investigation of locally dissipative acoustic metamaterials [25]. Locally resonant substructures were employed in [26, 27], showcasing the ability to use them within a host structure assembly to attain desired performance.

Simulation and numerical studies were carried out to verify the properties displayed from analytical methods. Comparison of attenuation mechanism for LRAM [12, 28] is one such example. Further, the existence of double-negativity [29] as viewed from dispersion curve was shown through simulations. Small-size sonic crystals displaying strong bandgaps were shown through numerical studies [30]. Numerical investigations performed by Narisetti et al by employing perturbation approach to reach the dispersion relation of 1-D discrete mass-spring chains with nonlinear stiffness included in the system enabled the study to postulate novel wave manipulation devices based on amplitude-dependence due to nonlinearity in the system [31]. This study proposes two possible devices backed with numerical investigations, showing amplitudedependent frequency isolator and a tunable narrow band-pass filter. As a design consideration, the diatomic chain with nonlinearity in the host chain, considered in this study may impose constraints on the device such as material selection for defined nonlinearity. This is because the approach to realize nonlinear stiffness in the chain may demand material nonlinearity, which places constraint by depending on choosing from the limited currently available constituent materials. Local nonlinear attachments in such case may have been a more favorable contender as such features can be created from the many different possibilities of realizing nonlinearity such as - geometric nonlinearity, nonlinearity through contact or via magnetic methods in addition to material nonlinearity. 


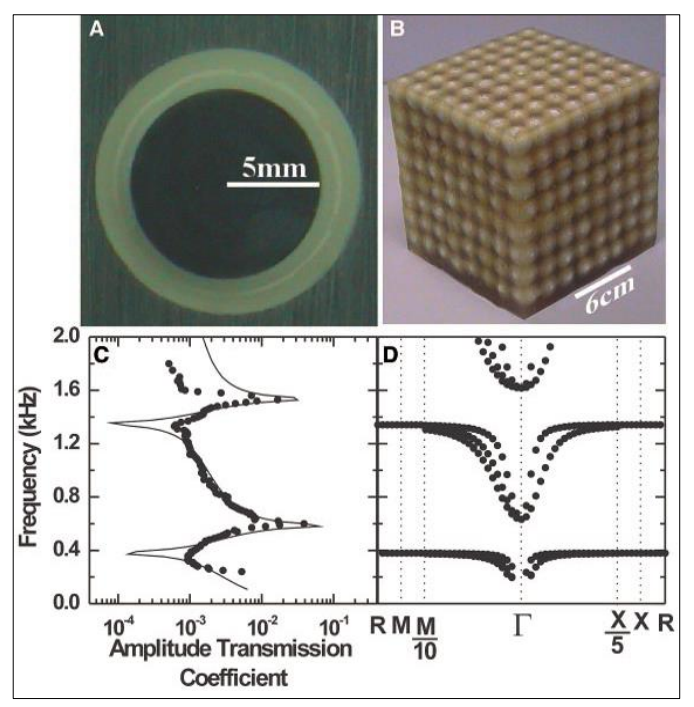

(a)

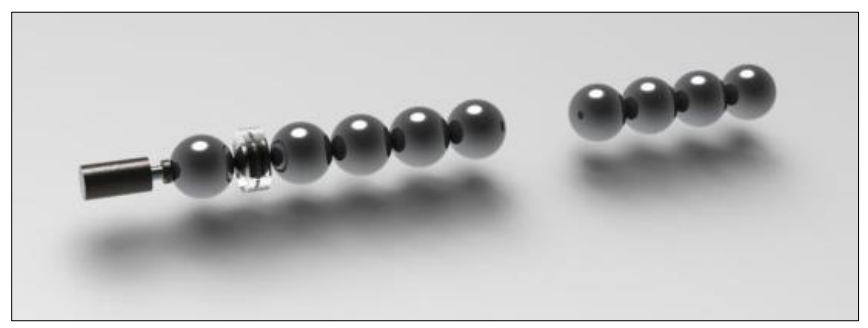

(b)

Figure 1. 1: Examples of metamaterials using (a) Locally resonant lead spheres [11] and (b)

Nonlinearity in granular chain [67].

For a long time, acoustic metamaterials inspired from their electromagnetic counterparts have been studied in numerous configurations. These studies employed different constituent elements to derive interesting phenomena, which are applicable to diversified scenarios. Association of instability, nonlinearity $[32,33]$, grounded springs and such have been studied to invoke certain characteristics so as to address specific engineering problems and applications. The ever growing advancement of technology has placed a continual demand on such innovative and specifically tailored materials; in which scenario, the study of inclusion of other possible elements is of such significance, to the extent of being indispensable. 


\subsection{Background}

Modern industrial age has employed systems such as machinery, automobile, space vehicles and so on, which are in constant interaction with dynamic forces inherent in their environment of application. Terrain topography, wind loading, ocean currents are a few examples of sources that induce dynamic load. This results in a high demand placed on effects that adversely affect the normal operation of machinery. Vibration is one such example. The constant interaction between system and environment; or even the internal working mechanisms itself may result in undesirable vibrations, which in some cases may jeopardize normal functioning. Such effects can be suppressed by careful design and integration of vibration isolators within the system, which by virtue of their natural frequency act as an energy sink for vibration in the close neighborhood of that frequency.

Another approach is to employ energy harvesting devices in cases wherein, dissipation of energy via stray vibrations is productively utilized. This was achieved by using the harmonic forcing as a means to sequester energy by employing tuned mass resonator [34].

\subsubsection{Negative and Nonlinear Stiffness}

In addition to the non-conventional negativity of mass, the other facet of metamaterials - negative stiffness is of particular significance. Stiffness is generally associated with the resistance offered to an active force and is known to directly influence the displacement of a particle from its position. Usually, this stiffness is positive, nonzero for structures to exhibit stable rigidity under load. However, some materials have been reported to exhibit negative stiffness under certain specific conditions [20]. This drives them to exhibit a large displacement under the action of even a slight force in a way to support the force and displace in its opposite direction. Whereas a system with a positive stiffness would oppose an externally applied loading, a system with negative stiffness would offer a decreasing resistance and can support the deformation even further [35]. Thus a negative stiffness system would experience larger deformation in contrast. 
The buckled beam is a classic example of negative stiffness. Consider the case of a bistable beam as shown in Figure 1. 2.

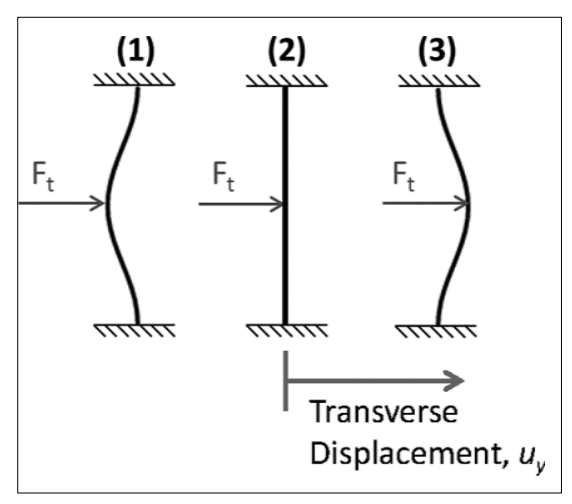

(a)

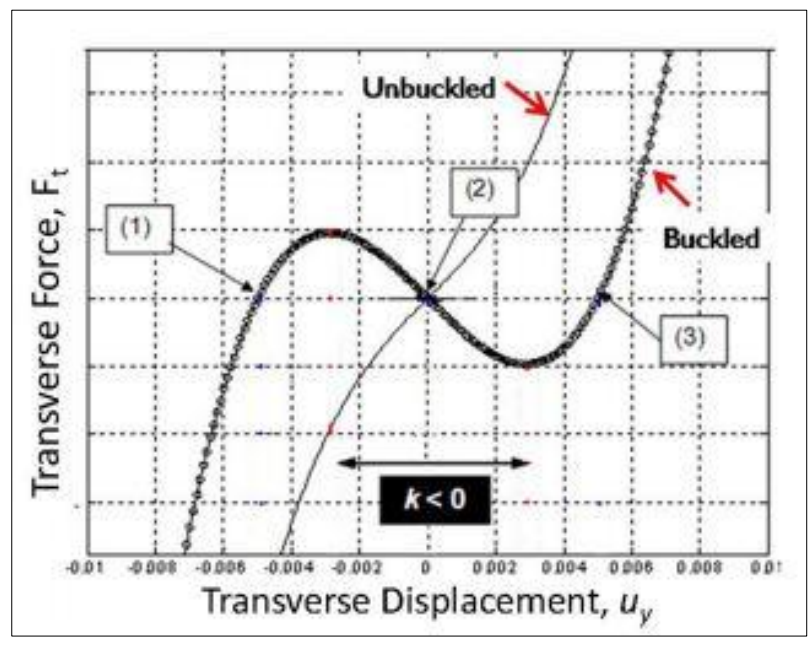

(b)

Figure 1. 2: $\quad$ One approach to obtain negative stiffness from bistable beam [38].

The beam is acted upon by an initial displacement given in the form of a compressive load and is held in that condition. This beam has three extreme configurations - two stable and one unstable position. Within the area of the unstable regime, even a significantly small load applied causes the beam to snap into one of its corresponding stable positions depending on the direction of application of force. Kashdan et al reported experimental evidence for negative stiffness induced 
by pre-buckling and pre-compression in a 3-D printed Nylon prototype [36]. A study by Li et al [37] presents a theoretical study of nonlinear effects in AM arising from simultaneously negative modulus and density with a design based on periodically distributed locally resonant features along a pipe. The amplitude-dependence of bandgaps on incident acoustic intensity is reported from the theoretical study. Dynamically pronounced behavior such as double negative pass-band is shown to be altered due to nonlinearities, resulting in switching of propagation behavior of the metamaterial. It has also been shown that negative stiffness improves vibration isolation characteristics of a system $[38,39]$.

Although capable of displaying unique dynamic behavior, the LRAM depends solely on the phenomenon of resonance. Also, only linear stiffness is assumed in the case of LRAM which in a physical system may not always be valid. Most systems traditionally treated as linear have some degree of nonlinearity in them which can be triggered by specific conditions like amplitude. For example, the basis of deriving standard beam deflection expression for a cantilever beam has its roots in the assumption of a thin beam. This is to avoid inclusion of nonlinear effects at high displacement. Similar is the case of the simple pendulum, where a small displacement assumption is made in order to keep the response of the system close to linear. To be technically correct such conditions are regarded as 'quasi-linear'.

With nonlinearity being an inherent property of physical systems, it carries some value to attempt to exploit this characteristic. The ability to explore nonlinearity can possibly result in the conception of new phenomena that may prove beneficial to solve engineering problems.

Studies carried out on structures including nonlinear stiffness enriched the domain of frequency dependent behavior of systems, which found particular potential application in vibration isolation and energy harvesting. The work from Ali Nayfeh [40] presents a comprehensive study of the behavior of nonlinear oscillations with cubic and quadratic nonlinearity. Systems with nonlinear stiffness have been known to provide improved vibration isolation capabilities over those with 
linear stiffness. A linear vibrating system can be rid of unwanted vibration by coupling it to a nonlinear attachment. This attachment, called 'Nonlinear Energy Sink' (NES) pumps out energy from a primary mode of the linear system into higher modes of itself, which are later rapidly dissipated due to losses. Cubic nonlinearities in such cases in tandem with zero linear stiffness were reported by Quinn et al [41]. They state that the dissipation of vibration energy is accelerated by the addition of cubically nonlinear stiffness. Further, it is shown that nearly $99 \%$ of the energy induced by a shock was dissipated to a specifically modified NES. This NES was obtained by a combination of negative linear stiffness and nonlinear stiffness components. A quasi-zero stiffness obtained from the negative stiffness of a Euler buckled beam is used to determine the vibration isolation characteristics of the passive nonlinear isolator by Liu et al [39].

Owing to the enrichment of dynamic response by the addition of nonlinear oscillator in an otherwise linear system, there has been an increased interest in the field of acoustic metamaterials that exhibit nonlinear response $[42,43]$. A perspective of looking at not just one or two degrees of freedom system for vibration isolation but considering a long lattice chain type system with the inclusion of nonlinearity emerged, to study wave propagation through these structures. Querying lattice chains with nonlinearities in different configurations gained recognition. Nonlinear dispersion relation in a periodic string was experimentally and analytically obtained by Manktelow et al [44]. The addition of nonlinearity to the now traditional LRAM was undertaken with the help of analytical methods by applying perturbation techniques such as multiple scale analysis. Narisetti et al [31] employed a perturbation approach to derive dispersion relation for one-dimensional nonlinear periodic structures with nonlinearity introduced in different configurations successively. The approach used assumed the first-order correction in frequency and provided evidence that introduction of cubic nonlinearity caused the propagation to become amplitude-dependent. In addition, dispersion relations obtained from varying configurations of lattice chains enabled theoretical postulation of novel amplitude and frequency dependent wave 
manipulating devices. Amplitude-dependent frequency isolator, which filters frequency content of an incident wave depending on its amplitude was modeled in a numerical setting. Another novel device implication includes a narrow band-pass filter which employs two hardening nonlinear chains of which one is grounded. The devices carry value based on their ability to filter out waves in conditions as mentioned but the key challenge lies in realizing a grounded chain in a physical system. Other device implications too, such as the one in the context of the current study - acoustic diode requires grounded stiffness for direction-bias in wave propagation. However, it was shown from simulations that by exploiting the amplitude-dependent wave modifying ability of the cubically nonlinear local attachments, a potential direction-bias waveguide can be assembled. Starting with simulations from [45] and investigations undertaken as part of this research, a possible design is postulated and subjected to experimental testing.

Unidirectional transmission of waves is of significant value in the area of acoustic switches, diodes or rectifiers to allow wave propagation only in a pre-decided direction. Waves propagating in the opposite direction are attenuated and ideally completely filtered. There has not been mention of experimentally fabricated in literature except [46], where periodic bistable members connected with magnetic links were built to mimic a system for strongly linear transition waves. A lattice consisting of 20 bistable elements supported by clamps on an aluminum rail forms the test-article which is an approximation of a 1-D discrete lattice chain.

The aforementioned studies motivated the investigation of new locally engineered configurations for AM. While on the one hand, the rise of specifically engineered materials and advancement in manufacturing technology relaxes the previously constrained design space of AM, on the other hand, there exist opportunities to explore new mechanical elements with unique dynamic responses. 


\subsubsection{The Inerter}

Inerters are mechanical elements that provide a force response proportional to the relative acceleration across them, are an attractive candidate for consideration in acoustic metamaterial structures. The force current analogy, where in forces substitute for currents and velocities for voltages, had an incompleteness considering the analogy for the non-grounded capacitor. The second terminal of mass was assumed to be at the centroid, analogous to second grounded terminal of the capacitor. The two-terminal or rather, the non-grounded capacitor which is an active component of electrical systems did not have a corresponding mechanical analogue. The mechanical analogue would have to relate the acceleration difference across its terminals to the applied force. The question is better emphasized through the following Figure 1. 3.

$$
\begin{aligned}
& \mathrm{F}=\mathrm{k}\left(x_{2}-x_{1}\right) \\
& \mathrm{F}=\mathrm{c}\left(\dot{x}_{2}-\dot{x}_{1}\right) \\
& \mathrm{F}=?\left(\ddot{x}_{2}-\ddot{x}_{1}\right)
\end{aligned}
$$

\section{Figure 1. 3: Missing parameter relating the relative acceleration across the element.}

Postulated by Smith [47] in 2002, the inerter completes the analogy between electrical circuits and mechanical networks on the basis of force-current analogy. Forming the mechanical analogue of the non-grounded capacitor from the electrical counterpart and completing the analogy as shown in Figure 1. 4. 


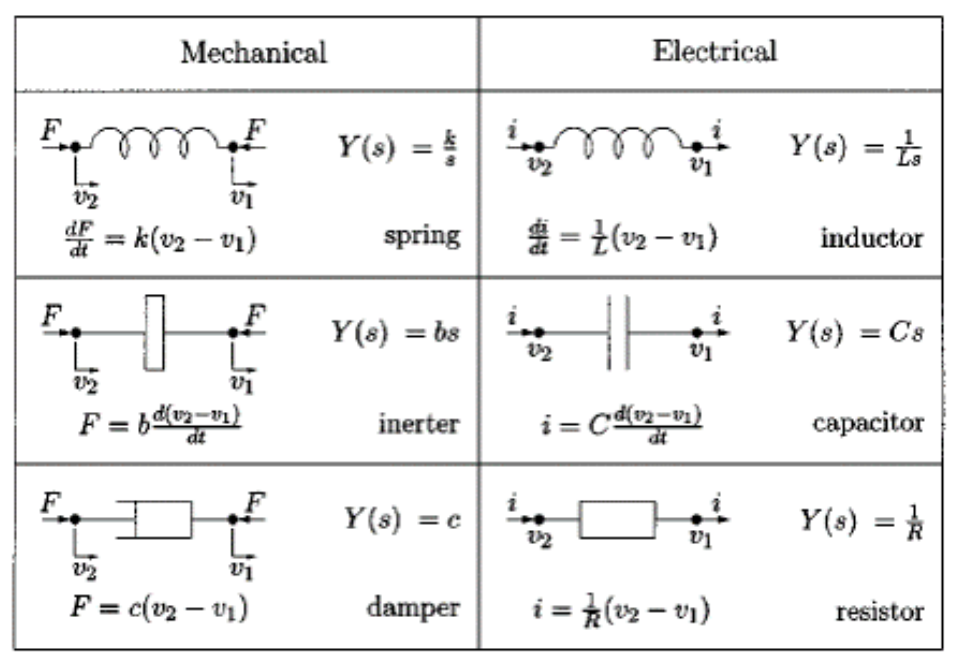

Figure 1. 4: Completed force-current analogy by including the inerter [48].

The inerters synthesized in the physical system were reported to display an amplified dynamic mass compared to the static, construction mass. With the unit same as that of mass, the inerter is denoted by the symbol ' $J$ '. A practically attainable ratio of inertance to device mass as high as 300 has been reported [47] through experimental investigations. Two designs proposed to practically fabricate inerters have been proposed by Chen et al [48] based on a Rack and Pinion mechanism and a ball screw assembly, as shown in Figure 1. 5 and Figure 1. 6 respectively.

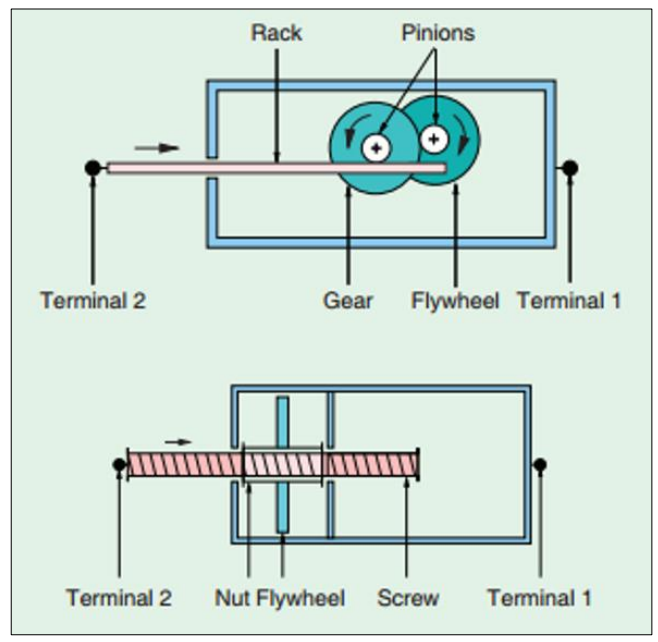

Figure 1. 5: Schematic representing a method to realize an inerter using rack and pinion mechanism [48]. 


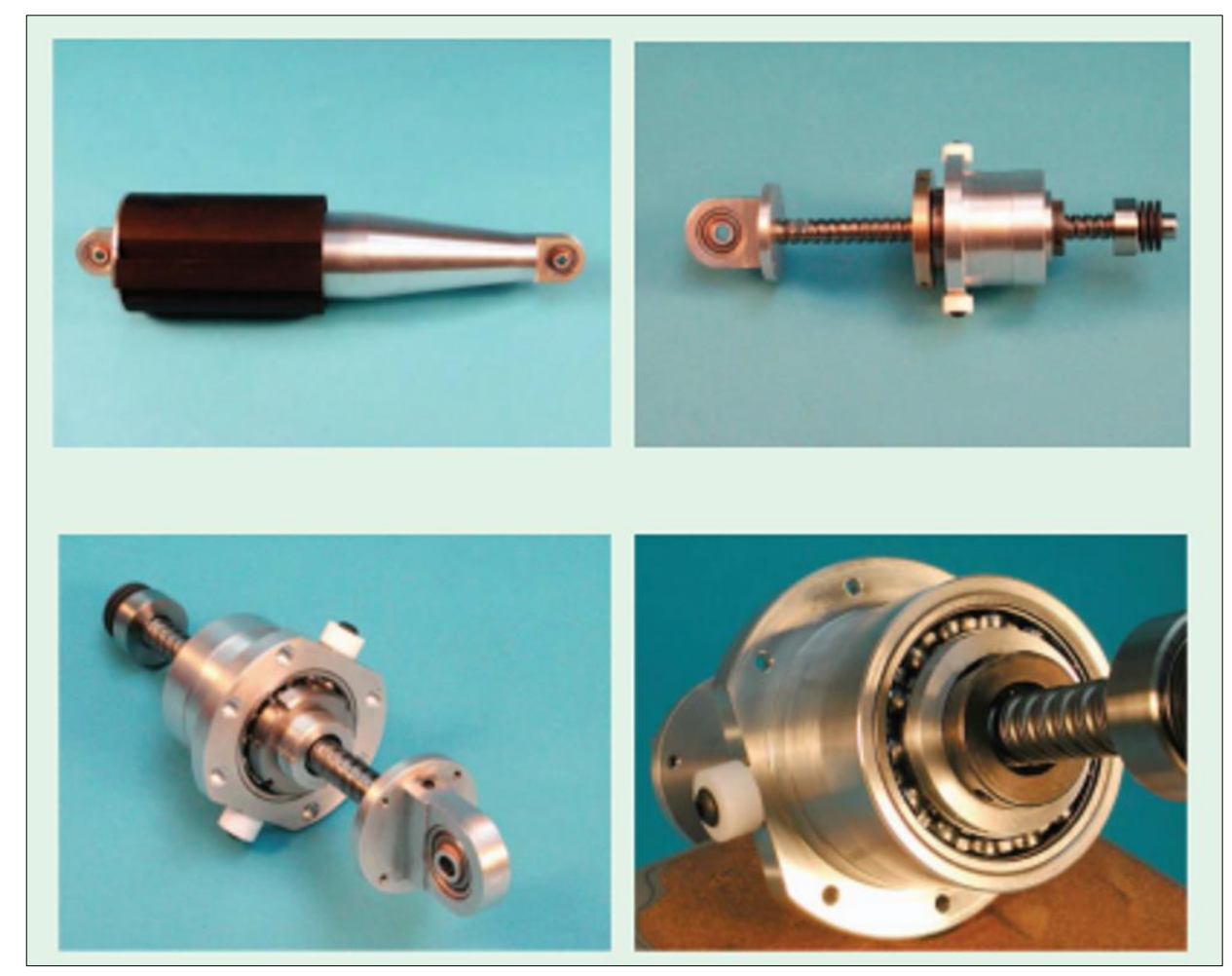

Figure 1. 6: An inerter device manufactured based on ball-screw type design [48].

Inerters have been studied as part of vibration isolation systems and their presence has been documented to result in improved performance over traditional vibration isolators and shock control systems. Chen et al [49] found that the natural frequencies of the single, dual and multiple degrees of freedom systems can be lowered due to the addition of inerter. An inerter-based device was proposed by Lazar et al [50], for vibration suppression in large-scale base-excited structures. The performance of vehicle suspension [51-53] and steering systems [54] utilizing inerters have also been studied. While inerters in vehicle passive suspension were studied earlier, $\mathrm{Hu}$ et al [55] extended the study by investigating the performance of different inerter-spring-damper configurations using an optimization approach including suspension displacement as a performance parameter simultaneously with other requirements such as tire grip and ride comfort. As a result, despite the improved performance offered, the limitation imposed by inerter was highlighted. Further, the benefits of employing inerters in semi-active suspension were studied by 
Chen et al [56] by investigating a combination of inerter based suspension strut and semi-active damper using simulations. They have reported improved overall performance by accounting for factors as in [55]. Single Degree of Freedom oscillator vibration absorbers employing inerters were reported to display superior performance compared to traditional Dynamic Vibration Absorbers for the same inertance-mass ratio [57]. From analytical optimization procedures, the former were found to have improved performance in addition to exhibiting the characteristic of having a larger mass ratio for smaller physical mass and also negated the need to add mass on the oscillator [57]. Inerters have also seen cutting edge commercial use, following their adoption under the moniker of ' $J$-damper' in Formula One cars. Experiments [58] to measure mechanical admittance functions of ball-screw and rack and pinion based inerter designs were conducted to gauge their relative performance. Extending the classical vibration control configuration of the tuned-mass-damper system by incorporating inerters, tuned-mass-damper-inerter systems [59] were shown to outperform them while remaining relatively lightweight. Numerical optimization [60] of vibration suppression devices with inerters predict 10 to $20 \%$ performance increase in a wider frequency band over traditional devices. Although the inerter was explored by many researchers in various domains with and majority researchers focusing on their application in vibration systems, the behavior of inertant systems in wave propagation has not been explored. This study investigates their effect by incorporating the inerter element in the infinitely long discrete 1-D lattice system in various configurations - introducing the inerter in both host and local attachments.

By adding nonlinearity, there was a significant impact on the harmonic response of an SDOF. Hence, the dynamic behavior of a linear system is enriched by the introduction of nonlinearity. It drives the system to display amplitude-dependent behavior. Further, the recently conceived inerter demonstrates an amplified dynamic mass. Nonlinearity and inerters are explored in 
different AM configurations through analytical techniques to investigate new phenomena. This is carried out to identify potential device implications.

\subsection{Definition of Objectives}

Motivated by the potential of acoustic metamaterials to display novel phenomena in the context of wave propagation, the objectives of this study have been identified as follows.

- Employ analytical and numerical methods to characterize the amplitude-dependent behavior of nonlinear AM, to explore the device implications.

- To conduct experimental verification of possibility of achieving passive direction-bias in elastic wave propagation.

- To characterize different inertant AM configurations in order to explore device implications.

\subsection{Chapter Overviews}

This thesis encompasses analytical, numerical and experimental investigations. Chapter 1 contains a general introduction to the field of acoustic metamaterials and attempts to draw out the advancement of research in this field. The possibility to add to the present body of research by investigating the effects of modifying the locally resonant type configurations is presented. In consequence, chapter 2 contains a simulation study proposing a direction-bias waveguide device. It introduces basic investigation of the nonlinear stiffness in a 1-DOF system, forming the basis for the design of a DBWG. Based on the simulation results, device implications are discussed. Among these implications, the direction-bias effect was chosen for its potential to make way to a fully functional acoustic diode. The direction-bias effect motivates the experimental study presented in Chapter 3. In this chapter, the process of translating the design parameters (obtained in chapter 2) to the experimental setup is illustrated elaborately. It is followed by a detailed description of the test apparatus, specimen manufacturing, and testing methodology. Chapter 4 is an analytical study performed by considering various configurations of inertant AM. The results 
obtained in this chapter are used to postulate some device applications, as wave manipulating devices. Finally, conclusions are presented in Chapter 5, along with a succinct account of recommendations for future are made. 


\section{CHAPTER II}

\section{NONLINEAR ACOUSTIC METAMATERIALS}

\subsection{Introduction}

Physical systems don't usually exhibit linear stiffness across the complete range of load application and tend to have nonlinearity in them. While this, in general, is disregarded during modeling (for simplicity), nonlinearity is rather the norm and linear stiffness is a special case. Although nonlinearity has been undesirable since they impart complex, chaotic and unpredictable behavior when introduced in a system, it can be exploited to obtain rich dynamic behavior targeted towards unlocking peculiar engineering applications.

The ability to manipulate cubic nonlinearity of a material is of particular interest because it may lead to an improved resolution in acoustic imaging [61] and directional bias in acoustic energy propagation [62]. There exists another point that interests the engineering community - energy dissipation and mechanical system of a material [35]. There usually is an inverse relation between these two properties wherein a stiffer material exhibits lesser dissipation and vice versa. It is thus of value to design composites which can both exhibit high stiffness with energy dissipation capabilities together. This can help develop devices which can isolate acoustical energy from a source or a structure.

Nonlinearity can be achieved in more than one way; it can be induced by one of or a combination of - material, geometric, inertial, constitutive, body force or friction. Constitutive nonlinearity is when stresses and strains are related by nonlinear function, unlike the linear relationship as given by 'Hooke's law', which itself holds for small strains. 
The relation between magnetic flux and field intensity renders two magnetic bodies to produce a force dependent nonlinearly on linear relative displacement. Further, the force of gravitation given by Newton is an example of a nonlinear system. Geometric nonlinearity is introduced in large strain or large rotation of solid continuum. Also possible is the case of a cantilever with varying geometry depending on displacement, which gives rise to a nonlinear stiffness. This particular type is adopted in the following work to experimentally design the desired nonlinearity. Another type of nonlinearity possible is from friction when the force of friction is a nonlinear function of displacement and velocity, as in dry friction or stick-slip friction.

Hence there are several ways of realizing nonlinearity in practice. Mann and Sims [63] utilized magnets to demonstrate a duffing-type nonlinearity to improve the performance of energy harvester over a wider range of frequencies. Jutte and Kota [64] propose a method to obtain predefined nonlinear stiffness derived from a geometrical arrangement of different stiffness elements.

To offset the limitations placed by engineering a material nonlinearity, a geometrical nonlinearity is catered. The nonlinearity is obtained by virtue of the nonlinear stiffness exhibited due to features such as contact in a system. A cantilever is designed that demonstrates nonlinear hardening behavior at higher amplitudes. It is due to varying effective length because of contact of the beam with the root profile. Hence, the term 'metamaterial' is justified. The property is being derived from the constituent unit-cell structure of a lattice as opposed to relying on the intrinsic material property itself.

In a study based on cubic nonlinearity in stiffness, the duffing oscillator cannot go unmentioned. A classic example of such nonlinearity, the duffing oscillator has been investigated extensively in relation to its dynamics after Georg Duffing. An in-depth analysis of this type of oscillator and other types of nonlinearities are dealt with by Ali Nayfeh and Cook in 'Nonlinear Oscillations' [40]. The effect of nonlinearity is to bend the displacement-frequency curve to bend away from 
the traditional $\Omega=1$ line on either side depending on the nature of stiffness - hardening or softening. When a softening type nonlinearity is introduced, the curve bends leftward and to right for a hardening case. When one notices that the response amplitude is higher for a larger domain of excitation frequencies, it becomes apparent that this is favorable for applications such as energy harvesting which solely depend on the high amplitude motion of oscillator at a set, the narrow frequency band in the close vicinity of the resonance frequency. For the very same reason, the duffing type oscillator finds a special place in the field of energy harvesting and vibration isolation. Several studies have postulated the use of the duffing oscillator for harvesting energy over a wider band of frequencies and some have provided experimental evidence of improved performance. The following numerical study has been carried out to both help understand the behavior of the cubic nonlinear oscillator and give a sense of completeness to the overall study.

\subsubsection{The Duffing Oscillator}

The objective of the following study is to obtain the response of a mass attached to a rigid support through a cubic nonlinear spring when the mass is acted upon by a harmonic force loading. The response is plotted in two different scenarios. In the first, frequency is incremented in steps from a lower bound up to a higher bound and holding a given frequency for some time before it is changed. In the second, the same procedure is applied in the opposite sense - the frequencies are decremented from higher to lower bound. The simulation was set up in Abaqus with the parameters chosen as $-\left[m, k_{0}, k_{n}, c, f\right]=[1,1000,100,1,200]$. The excitation frequency is incremented from $4.75 \mathrm{~Hz}$ to $8.25 \mathrm{~Hz}$ in steps of $0.25 \mathrm{~Hz}$, holding each frequency for the $20 \mathrm{~s}$ to ensure attainment of steady state. The natural frequency of the system, $\omega_{0}=5.03 \mathrm{~Hz}$ is included within the excitation. The resulting displacement of mass in both cases is plotted against the respective frequency of forcing and compared. It can be seen that on incrementing the forcing frequency, the response follows the path marked with circles in Figure 2. 5. The case with a decrement in frequency causes the response to follow the path marked with an asterisk. The 
analytical result for the same case with only linear stiffness is plotted to showcase the apparent difference in response in comparison in Figure 2. 5. A wider frequency range of high amplitude response of the duffing oscillator case is particularly worth noting here, which makes it desirable for applications such as energy harvesting over a wider frequency range.

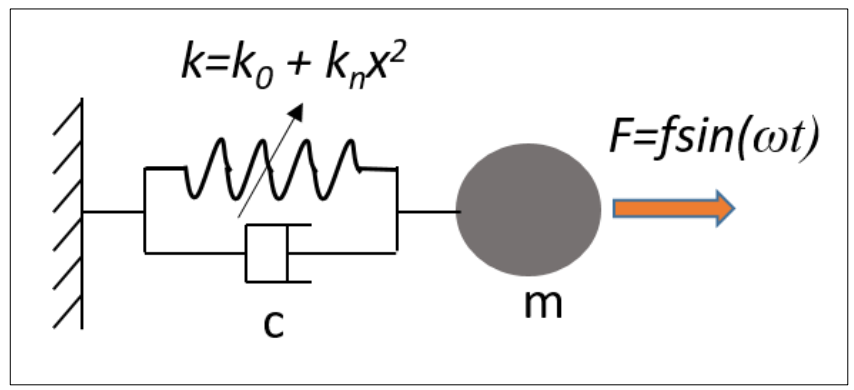

Figure 2. 1: Schematic diagram of the cubic nonlinear duffing oscillator used for simulation in Abaqus.

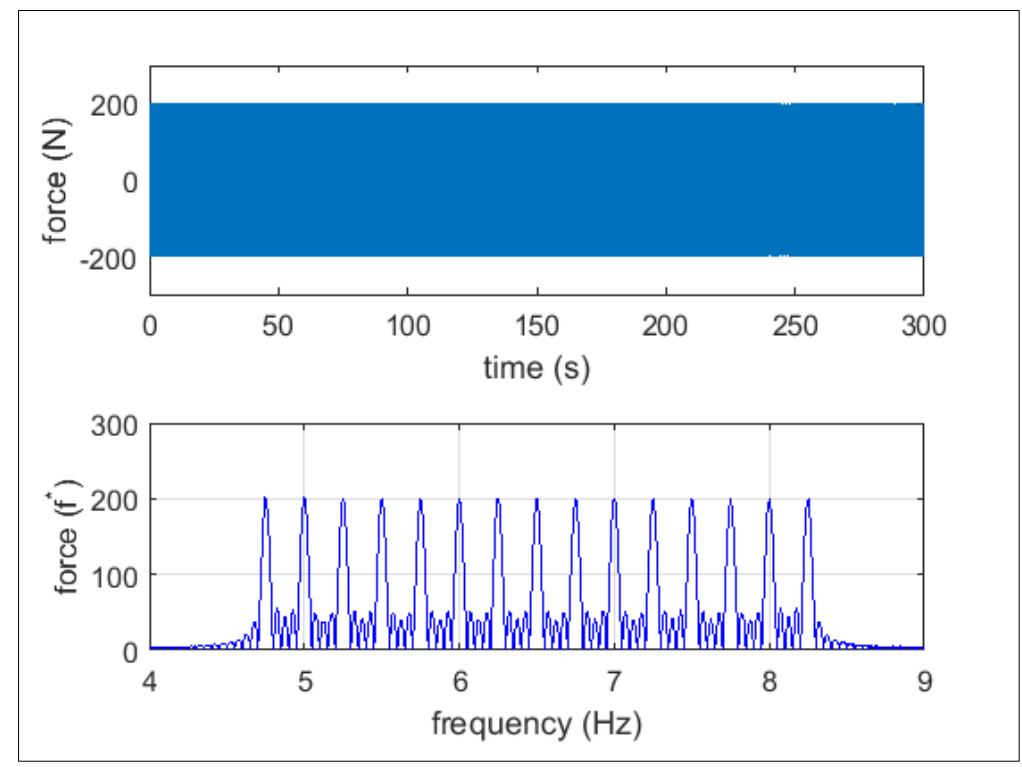

Figure 2. 2: Constant amplitude harmonic forcing with incrementally varying frequency. 


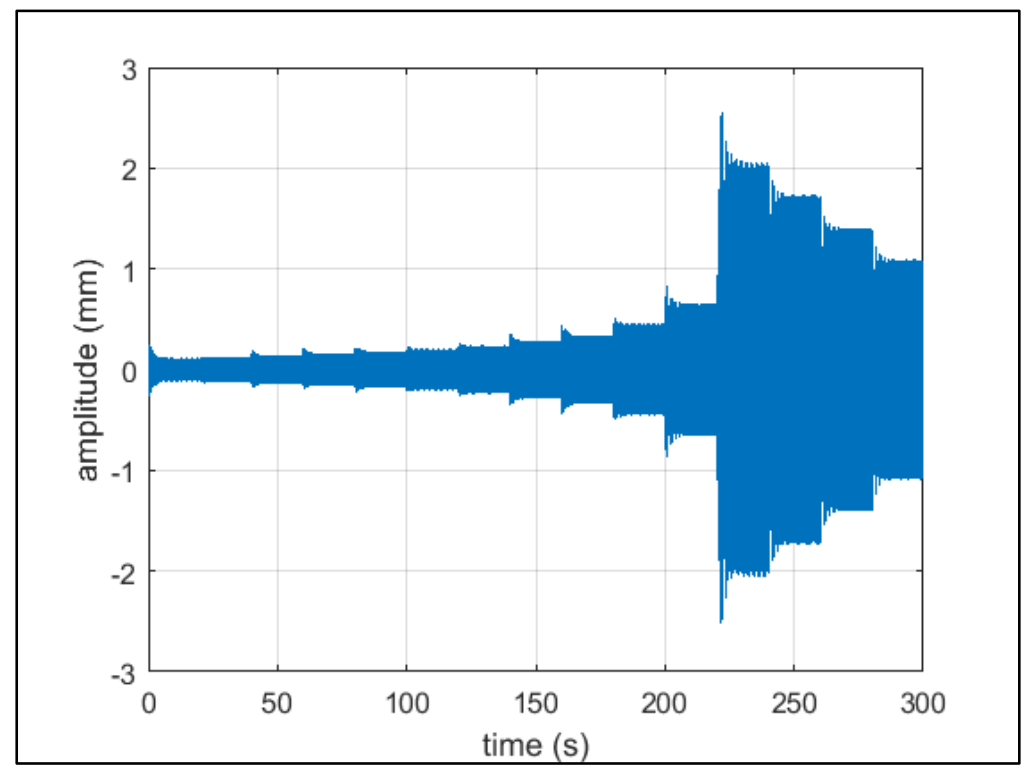

Figure 2. 3: Displacement time history of mass with decreasing frequency forcing.

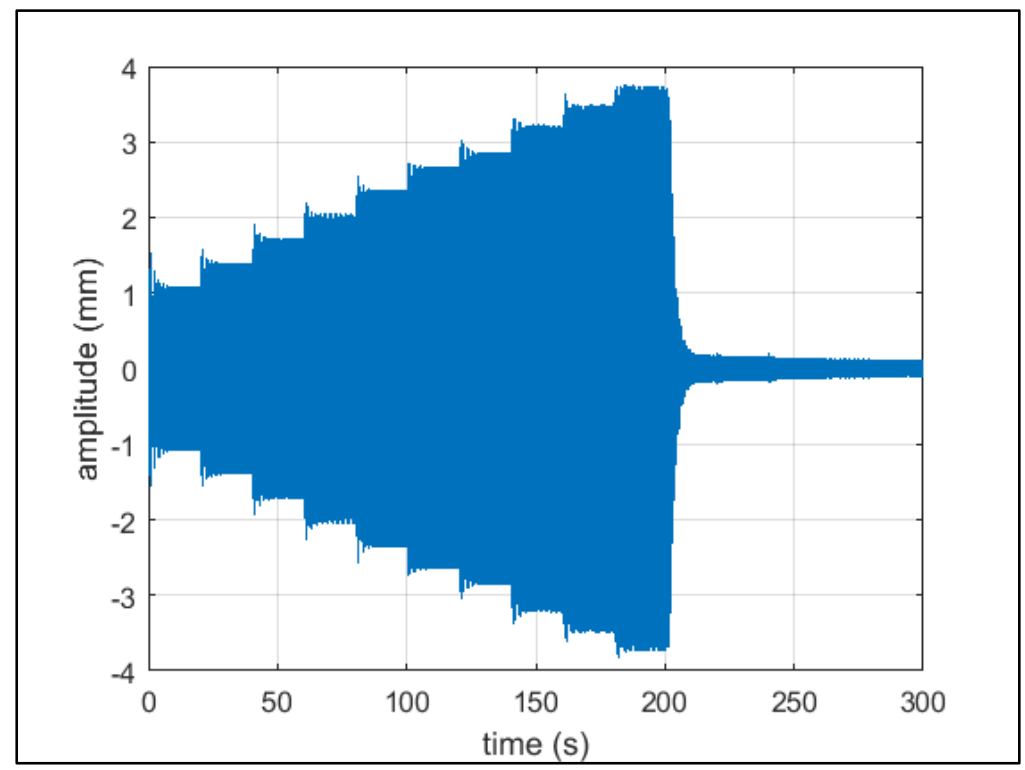

Figure 2. 4: Displacement time history of mass with increasing frequency forcing. 


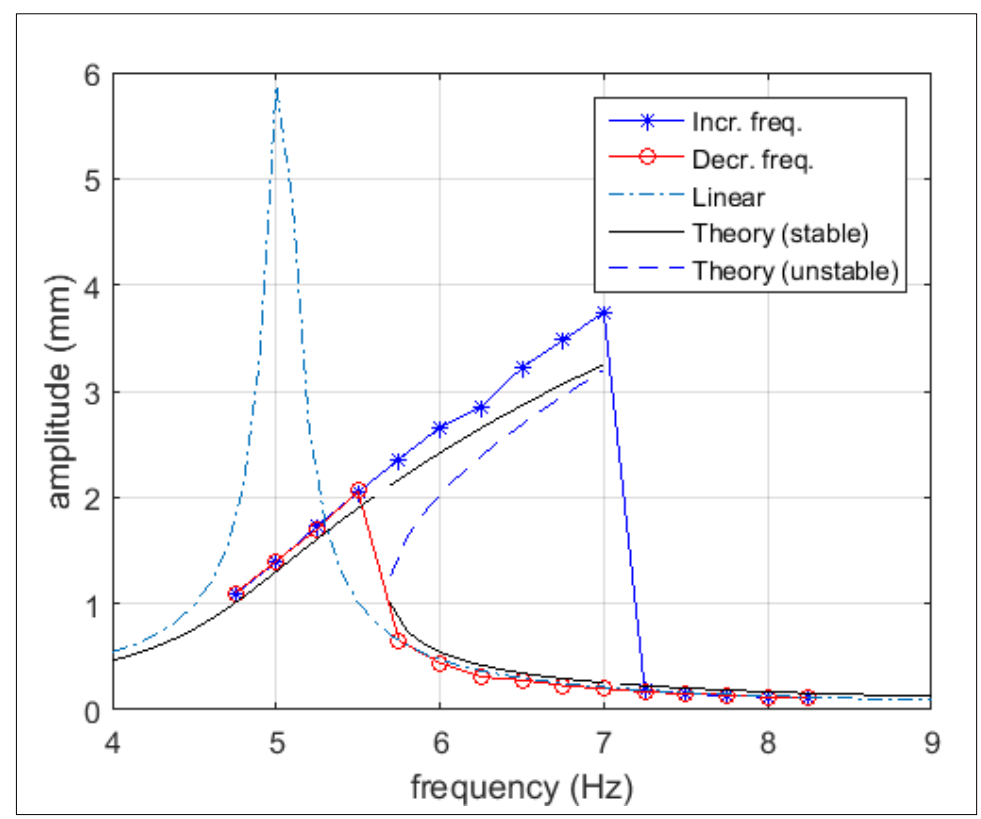

Figure 2. 5: Displacement plotted as a function of amplitude in the case of increasing and decreasing forcing frequency. The analytical response of the system with linear stiffness is plotted for comparison.

The characteristic of the Duffing oscillator response is the three-valued solution. This behavior is captured in the Figure 2. 5 through the theoretically obtained curve. The simulated response reveals that the response exhibited depends on the direction of approach in frequency in the threevalued region. The response latches onto either branch depending on direction of approach of excitation frequency while the third solution is unstable and is not realizable in the simulation.

It is worthwhile to throw light on the observation that the more desirable high amplitude response occurs depending on the direction of approaching this regime. This 'jump phenomenon' is a characteristic of the cubically nonlinear type duffing oscillator and though the maximum amplitude attained by this system is lesser compared to the linear counterpart, the advantage lies in the fact that the higher amplitude is retained for a broader band of frequency. 


\subsection{Analytical Model}

A one-dimensional infinite mass-spring chain is considered with periodic local mass attachment via a nonlinear stiffness spring. The chain is assumed to have been acted upon by a harmonic forcing, $\mathrm{F}(\mathrm{t})$ on one end and is studied for wave propagation characteristics. The local attachments are assumed to have cubic nonlinearity given by.

$$
k=k_{0}+k_{n} x^{2}
$$

An infinite periodic chain with a repeating unit cell is considered. This unit cell constitutes of 2 masses connected by non-linear springs as shown in Figure 2. 6

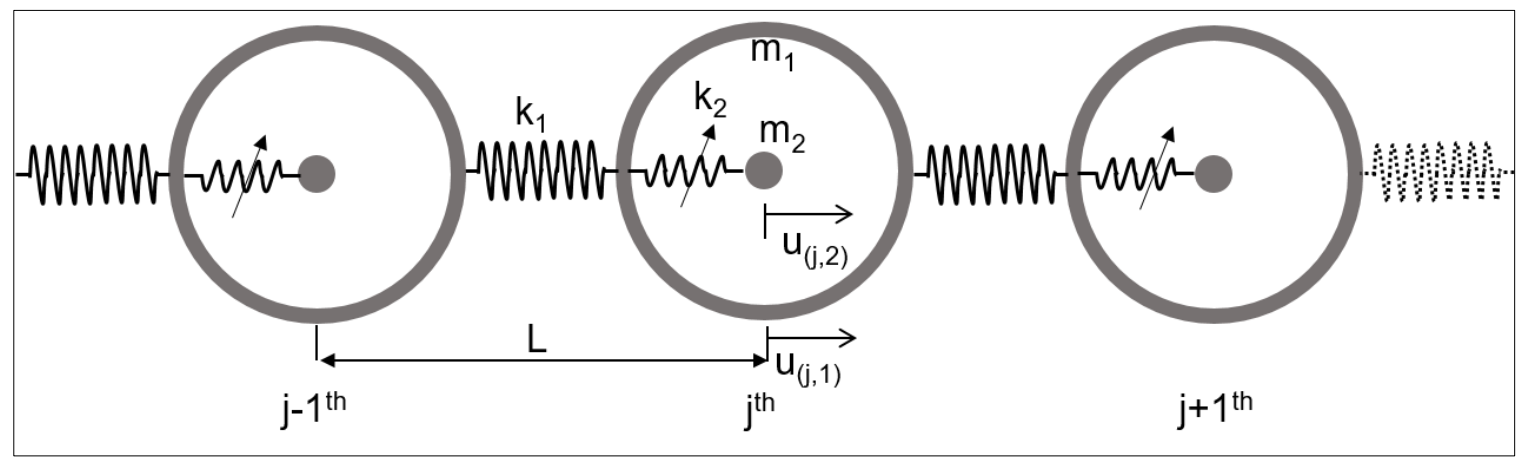

Figure 2. 6: Discrete mass-spring lattice model for an AM with local nonlinear attachment.

$\varepsilon$ represents a small parameter called as perturbation parameter. Equations of motion governing displacements in the $j^{\text {th }}$ unit cell can be expressed as

$$
\begin{gathered}
m_{1} \ddot{u}_{j, 1}+k_{1}\left(2 u_{j, 1}-u_{j+1,1}-u_{j-1,1}\right)+k_{2}\left(u_{j, 1}-u_{j, 2}\right)+\varepsilon \Gamma\left(u_{j, 1}-u_{j, 2}\right)^{3}=0 \\
m_{2} \ddot{u}_{j, 2}+k_{1}\left(u_{j, 2}-u_{j, 1}\right)+\varepsilon \Gamma\left(u_{j, 2}-u_{j, 1}\right)^{3}=0
\end{gathered}
$$

Based on Lindstedt- Poincare perturbation technique, dispersion relations corrected up to second order are solved. Displacements of each mass and plane wave frequency are first stated as asymptotic series.

$$
\omega=\omega_{0}+\varepsilon \omega_{1}+O\left(\varepsilon^{2}\right)
$$




$$
u=u^{(0)}+\varepsilon u^{(1)}+O\left(\varepsilon^{2}\right)
$$

Equation 2.1 is substituted in Equation 2.2 and 2.3 and $u_{j}$ is assumed harmonic. Then the first two ordered equations obtained are as follows,

$$
\begin{gathered}
\varepsilon^{0}: \alpha \bar{\omega}_{0}^{2} \ddot{u}_{j, 1}^{(0)}+\frac{k_{1}}{k_{2}}\left(2 u_{j, 1}^{(0)}-u_{j-1,1}^{(0)}-u_{j+1,1}^{(0)}\right)+\left(u_{j, 1}^{(0)}-u_{j, 2}^{(0)}\right)=0 \\
\varepsilon^{1}: \alpha\left(\bar{\omega}_{0}^{2} \ddot{u}_{j, 2}^{(1)}+2 \bar{\omega}_{0} \bar{\omega}_{1} \ddot{u}_{j, 1}^{(0)}\right)+\frac{k_{1}}{k_{2}}\left(2 u_{j, 1}^{(1)}-u_{j-1,1}^{(1)}-u_{j+1,1}^{(1)}\right)+\left(u_{j, 1}^{(1)}-u_{j, 2}^{(1)}\right) \\
+\bar{\Gamma}\left(u_{j, 1}^{(0)}-u_{j, 2}^{(0)}\right)^{3}=0
\end{gathered}
$$

A plane wave solution is assumed for the $\varepsilon^{0}$ equation. Considering $n^{\text {th }}$ generalized coordinates, the displacement in the $j^{\text {th }}$ cell is expressed as,

$$
\begin{gathered}
\dot{u}_{j, 1}^{(0)}=i \frac{A_{1}^{(0)}}{2} e^{-i\left(k_{j} a\right)} e^{-i \Gamma}+(-i) \frac{\bar{A}_{1}^{(0)}}{2} e^{-i\left(k_{j} a\right)} e^{-i \Gamma} \\
\ddot{u}_{j, 1}^{(0)}=-\frac{A_{1}^{(0)}}{2} e^{i\left(k_{j} a\right)} e^{i \Gamma}-\frac{\bar{A}_{1}^{(0)}}{2} e^{-i\left(k_{j} a\right)} e^{-i \Gamma}
\end{gathered}
$$

Where $k$ represents the wavenumber, ' $a$ ' denotes the separation of two $m_{l}$. Given the cell number $j$ and mass position index $n$ inside unit cell, $s(j, n)$ is a function that returns global mass position index (integer).

$\mu$ is a wavenumber, where $\mu=k a$. The solution to the $\varepsilon^{0}$ - order equation results in a dispersion relationship for the lattice.

For order- $\varepsilon^{1}$, the updated equation is given by,

$$
\begin{aligned}
& \alpha\left(\bar{\omega}_{0}^{2}\left(-\ddot{u}_{j, 2}^{(1)}\right)+2 \bar{\omega}_{0} \bar{\omega}_{1}\left(-\ddot{u}_{j, 1}^{(0)}\right)\right)+\frac{K_{1}}{K_{2}}\left(2 u_{j, 1}^{(1)}-u_{j-1,1}^{(1)}-u_{j+1,1}^{(1)}\right)+\left(u_{j, 1}^{(1)}-u_{j, 2}^{(1)}\right) \\
& +\bar{\Gamma}\left(u_{j, 1}^{(0)}-u_{j, 2}^{(0)}\right)^{3}=0
\end{aligned}
$$

The equation shown above can be written as 


$$
c_{1} e^{i\left(k_{j} a+\Gamma\right)}+c_{3} e^{3 i\left(k_{j} a+\Gamma\right)}+c . c=0
$$

All terms in the above equation that occur with functional form $e^{i k s(j, n) a}$ behave to force the updated $\varepsilon^{l}$ - order equation at resonance. This leads to unbounded solutions. These terms are recognized as secular terms. In order to obtain a bounded solution at order- $\varepsilon^{l}$, these terms must be eliminated.

$$
c_{1}=2 A_{1}^{(0)} \bar{\omega}_{0} \bar{\omega}_{1}-\bar{\Gamma}\left(1-\frac{1}{1-\bar{\omega}_{0}^{2}}\right)^{3}\left(\frac{3}{4} 2 A_{1}^{(0)^{2}} \bar{A}_{1}^{(0)}\right)
$$

This returns an expression for the frequency correction $\mathrm{w}_{1}$ in terms of $A^{(0)}$ and $\mu$.

Substituting the assumed solution and parameters, solving the system of equations by dropping the higher order terms in expansion and equating the constituents of the significant parameter to zero yields

$$
\begin{gathered}
\bar{\omega}_{1}=\frac{3 \bar{\Gamma}\left|A_{1}\right|^{2}}{8 \alpha \bar{\omega}_{0}}\left(1-\frac{1}{\left(1-\bar{\omega}_{0}^{2}\right)}\right)^{3} \\
\omega=\omega_{0}+\varepsilon \frac{3 \bar{\Gamma}\left|A_{1}\right|^{2}}{8 \alpha \bar{\omega}_{0}}\left(1-\frac{1}{\left(1-\bar{\omega}_{0}^{2}\right)}\right)^{3}
\end{gathered}
$$

Dispersion relation of the system is given by Equation 4.3 described in detail in section 4.2.1. The dispersion relation with first order correction can be plotted by substituting the value of $\omega$ from Equation 2.14 into the equation. The obtained result when plotted shows the effect of perturbing the system with the perturbation parameter $\varepsilon$. Even for a very small value of $\varepsilon$, it can be seen that having a cubically nonlinear hardening stiffness as in this case, the bandgap is slightly smaller than the case with linear stiffness in the local attachment. Furthermore, for this particular case of modified LRAM, upper bound of bandgap experiences a larger shift than the lower bound which for an LRAM is $\omega=\omega_{0}$. 
It can be shown that the propagation of a system with nonlinear hardening stiffness has an amplitude-dependence and that with increasing amplitude of excitation, the band gap is narrowed. Thus in the case of hardening cubic nonlinearity, the cutoff of bandgap increases with increase in the amplitude of excitation. The perturbation technique helps analyze similar systems employing nonlinearities to a chosen degree of approximation. A few cases that have already been studied [31] in the past by this method include - a monoatomic mass-spring chain and nonlinear springs, nonlinear diatomic chain, nonlinear stiffness in host chain with linear local attachment. Resulting dispersion relation obtained from the technique applied on an established discrete mass-spring lattice with local nonlinear attachment are plotted to show the shift in bandgap limits due to the introduced cubic nonlinearity. With a nonlinear softening (NLS) local attachment the lower bound of the band gap is lowered while by introducing an NLH, on the other hand, causes the bandgap to narrow down compared to the limiting case of LRAM. The upper bound is not as significantly affected by the introduction of NLS or NLH and hence it provides the potential to tune the AM to have a narrow band or specific frequency filtering characteristics.

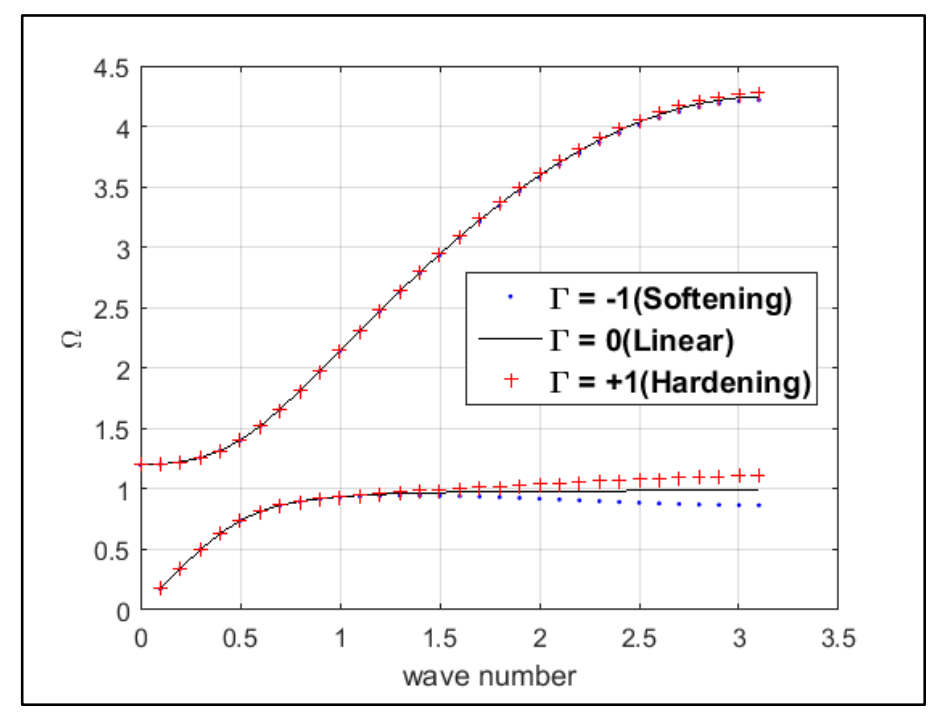

Figure 2. 7: Dispersion curves obtained by perturbation method for LRAM type lattice with softening and hardening type nonlinearity in the local attachment. The excitation frequency normalized with local resonance frequency is plotted against the wave number. 


\subsection{Simulations}

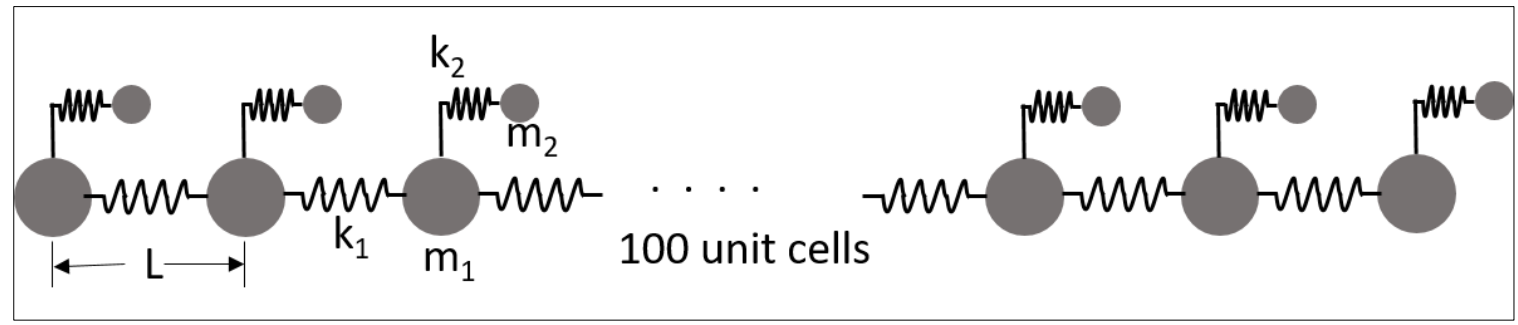

Figure 2. 8: Simulation model set up in Abaqus for LRAM.

\subsubsection{Locally Resonant Acoustic Metamaterial (LRAM)}

Consider a 1-D representation of an LRAM which is modeled as an infinite mass-spring chain with local resonant attachment. The system consists of a host mass $m_{l}$, attached via springs of stiffness $k_{1}$. A mass $m_{2}$ is attached to $m_{1}$ through a spring $k_{2}$. A schematic of this system is shown in Figure 2. 8. To obtain the wave propagation characteristics of this system, the equations of motion for the two masses are solved for a harmonic forcing. The dispersion relation for the LRAM is given in Equation 4.3, the technique to obtain this equation is mentioned in [12] is described in section 4.2.1

The dispersion relation displays a bandgap region, which is introduced due to the introduction of local attachment. It can be shown that a wave at a frequency within this bandgap experiences spatial attenuation. This is because the wave number at this frequency becomes complex. The frequency interval that marks the bandgap limits is given by the Equation 4.2. The limits can be expressed as follows

$$
\omega_{0}<\omega<\omega_{0} \sqrt{1+m_{2} / m_{1}}
$$

A simulation model consisting of 100 unit cells of the LRAM is set up in Abaqus by using pointmass and stiffness elements available in the program. A harmonic excitation is applied on $1^{\text {st }}$ mass and the displacement at the $100^{\text {th }}$ unit cell is recorded. These are referred to as 'input' and 
'output' respectively. The displacement of $m_{l}$ is taken into consideration to do this. The system is assumed is assigned with a parametric setting as shown below. Using these parameters, the bandgap limits can be obtained as $[1115 \mathrm{~Hz}-1260 \mathrm{~Hz}]$. An excitation frequency chosen out of this range should propagate while that within these bounds should experience attenuation.

Hence $500 \mathrm{~Hz}$ and $1120 \mathrm{~Hz}$ are chosen as the former lies outside and the latter within the bandgap. The system parameters are chosen with the intent to translate the system to an experimental test-article. Hence, manufacturing constraints are taken into consideration. The attachment mass and stiffness values are chosen so as to be realized in a physical scenario. The output and input displacements are plotted as shown in Figure 2.9. The simulations show a good agreement with the theoretically established band gap predictions.

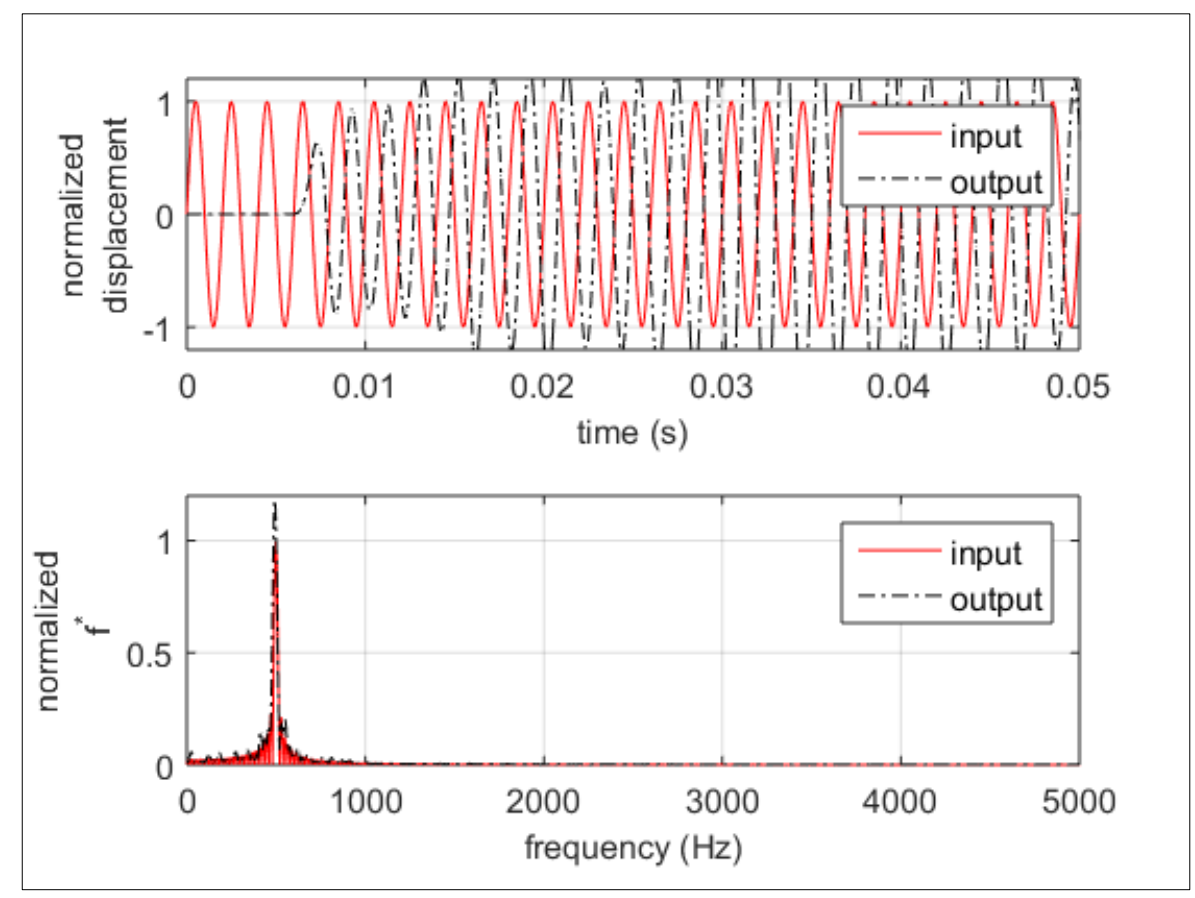

(a) 


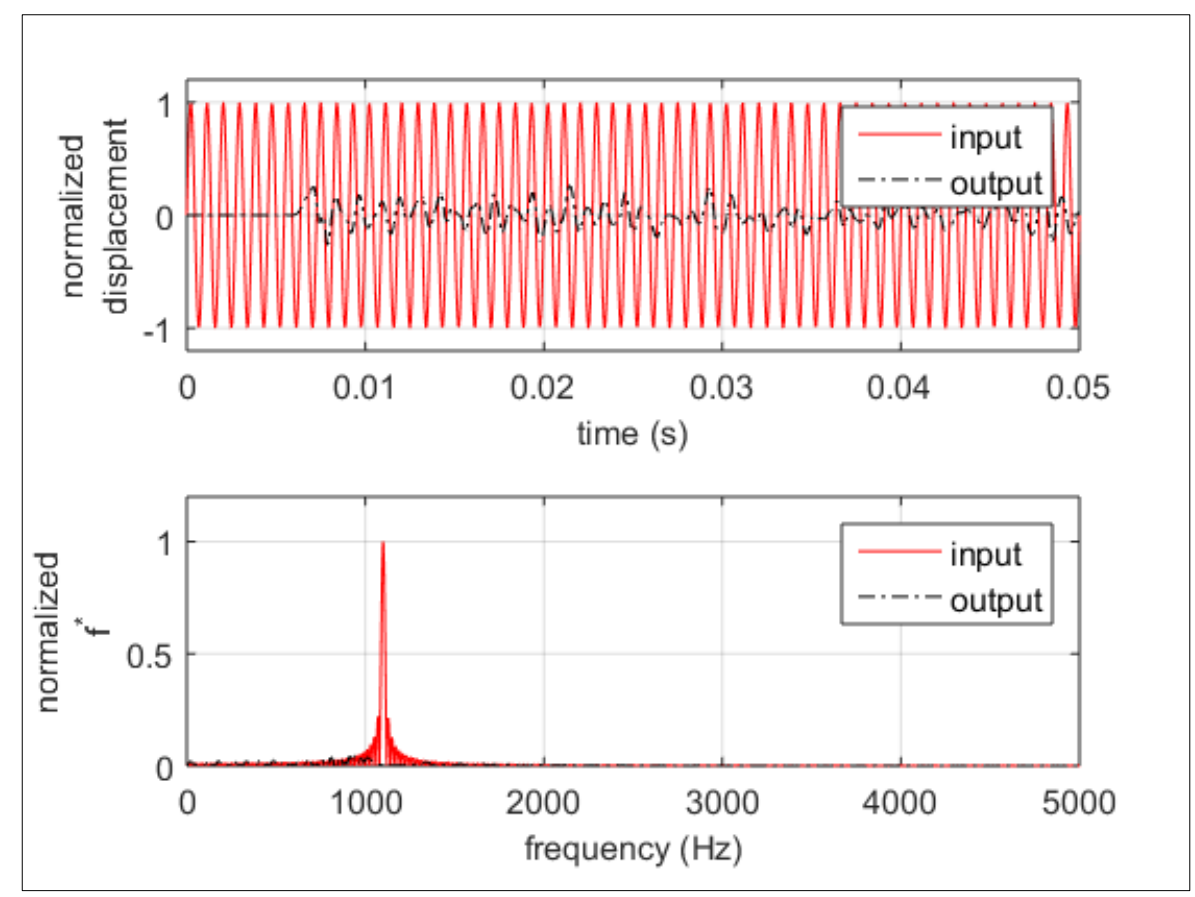

(b)

Figure 2. 9: Demonstration of bandgap effect through simulations in an LRAM.

\subsubsection{Nonlinear Local Attachments}

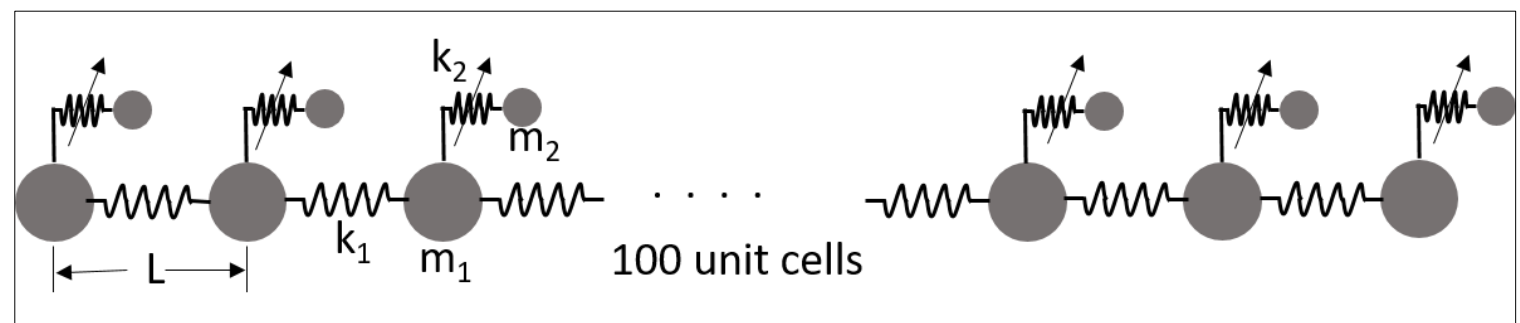

Figure 2. 10: Simulation model set up in Abaqus for LRAM.

A local nonlinear attachment similar to that discussed in section 4.2 is considered here for numerical investigation. The parameters chosen for this system similar to that of LRAM were chosen with manufacturing constraints in view. Nonlinearity is a function of displacement amplitude. Hence, $k_{n}$ for the system is chosen such that at sufficiently small displacement, it behaves close to an LRAM as nonlinearities are not triggered. Therefore, at low amplitude, the bandgap limits of this NLAM can be obtained from Equation 4.2 as [600 Hz - $980 \mathrm{~Hz}]$. 
A simulation model similar to LRAM, as shown in Figure 2. 10 with 100 unit cells is set up in this case with the following system parameters.

$$
\left[m_{1}, m_{2}, k_{1}, k_{2}, k_{n}\right]=[1.67 E-3,2.84 E-3,2.43 E 6,40382.45,1 E 11]
$$

Keeping the condition for triggering of nonlinearity in view, a low amplitude $(0.01 \mathrm{~mm})$ single frequency harmonic excitation is applied at $1^{\text {st }}$ mass and resulting displacement of $100^{\text {th }}$ mass is recorded. The output and input displacement plots are shown in Figure 2. 11. The simulations show a marked attenuation at $606 \mathrm{~Hz}$, which lies just within the bandgap while $500 \mathrm{~Hz}$ shows propagation as it lies below in the predicted propagation zone.

At the same frequencies, a high amplitude $(1 \mathrm{~mm})$ excitation is applied to observe the effect of nonlinearity, which is triggered due to increasing the amplitude. The plots obtained for this case are shown in Figure 2. 12. It can be seen that at high amplitude, the response at $606 \mathrm{~Hz}$ switches to propagation. This is a consequence of narrowing of bandgap as predicted in section 2.2 and shown in Figure 2. 7. The reason for such phenomenon is nonlinearity, which renders the response of a system to become amplitude-dependent.

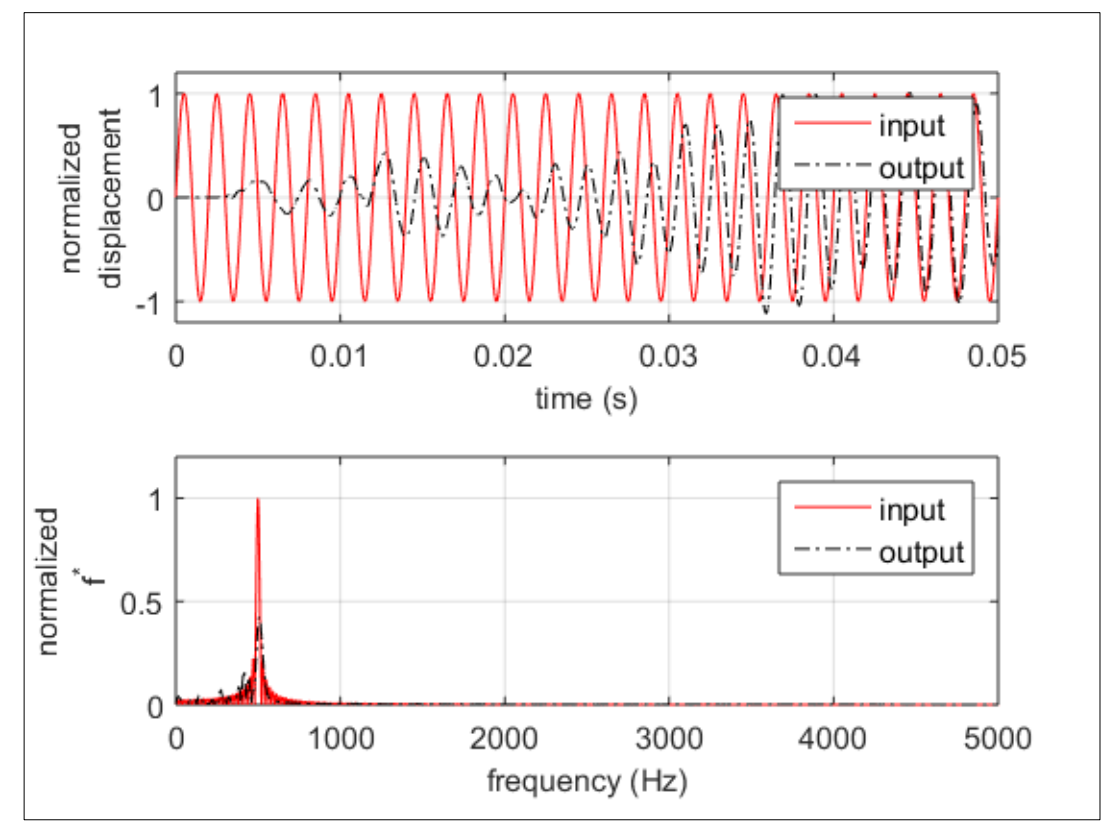

(a) 


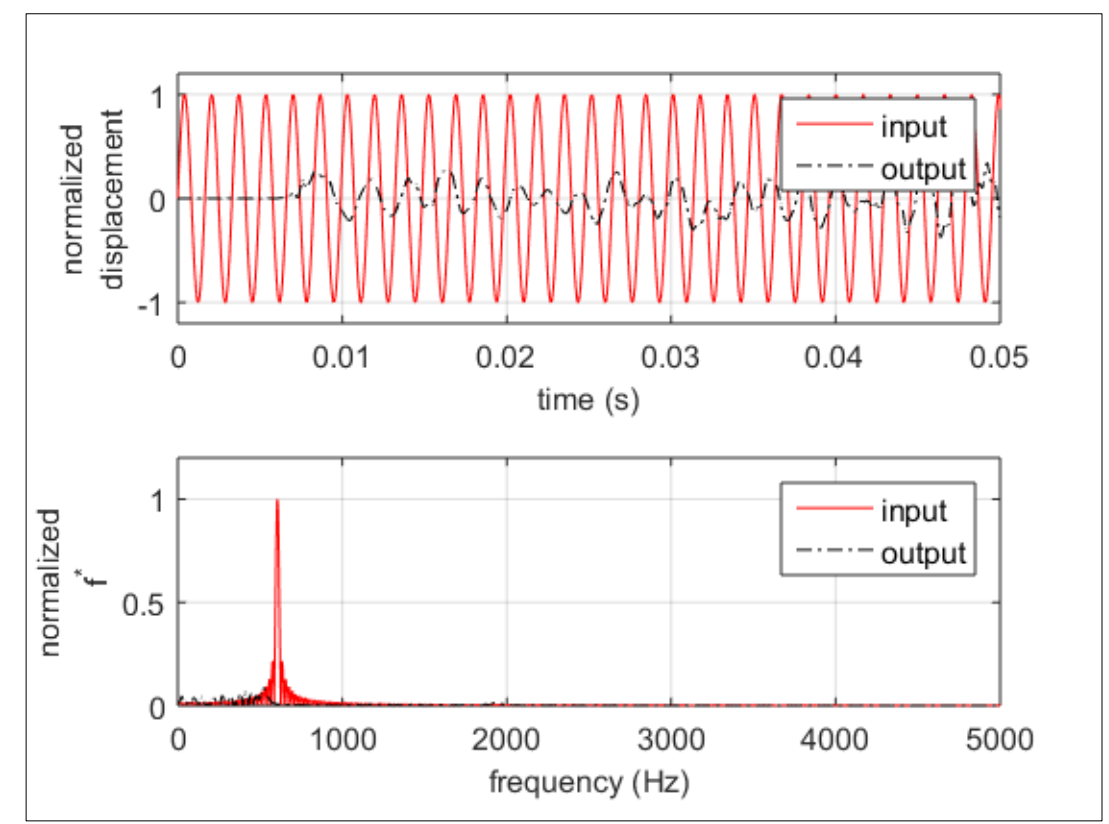

Figure 2. 11: Demonstration of bandgap effect through simulations for NLAM.

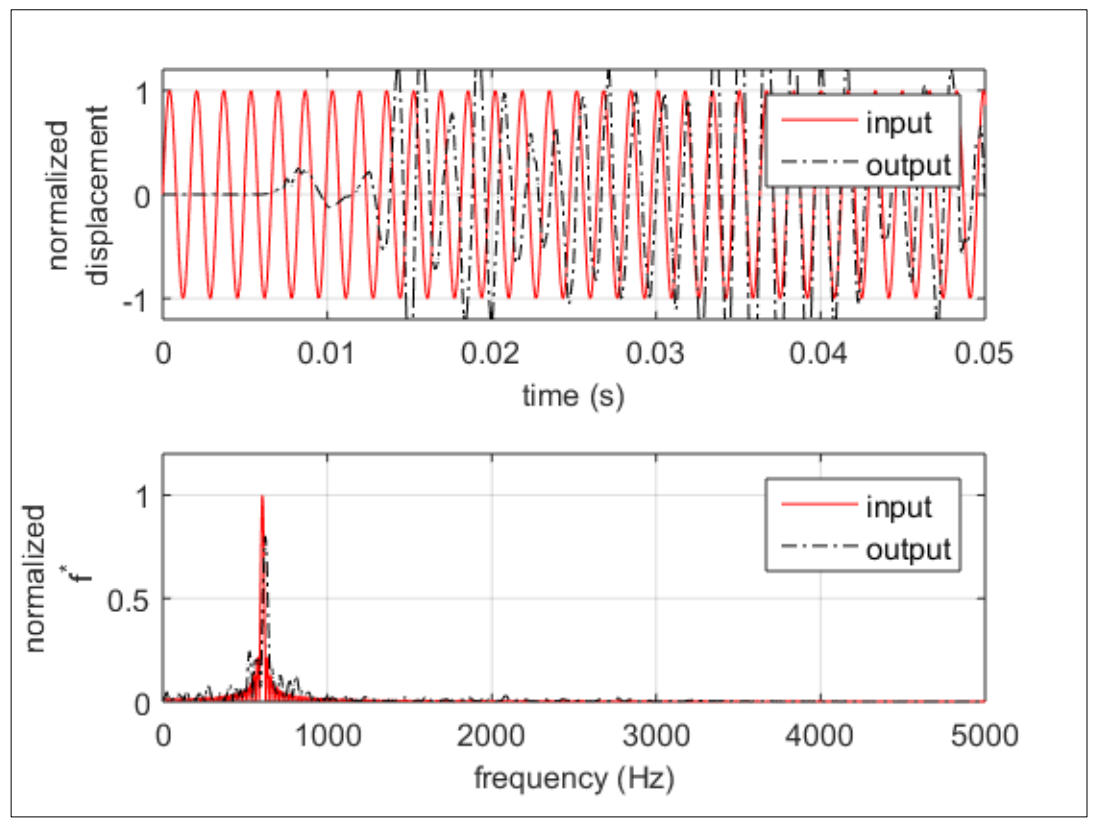

Figure 2. 12: Amplitude-dependent propagation due to nonlinearity.

The effect of high amplitude excitation through the NLAM is shown in Figure 2. 13. The attenuation at low amplitudes is expected as nonlinearities are not triggered and the resonators still behave as quasi-linear oscillators. But due to nonlinearities being triggered at a higher 
amplitude $(\mathrm{A}=1 \mathrm{E}-3)$, we see a higher order behavior being introduced in the system causing the output amplitude to be larger at certain times than the input amplitude. This switching from attenuation to propagation can be attributed to the shift of lower limit of the bandgap as shown in Figure 2. 5. A detailed analysis of the effect of nonlinearity and amplitude on nonlinear AM has been undertaken in [45].

Further investigations were carried out on the system by varying the excitation frequency. This is done by varying the frequencies in increments away from the local resonance frequency. Therefore, these frequencies of excitation are referred to, as normalized values. An interesting amplitude-dependent behavior is observed at $\Omega=1.9$. The displacement response for input and output obtained for this case is plotted in Figure 2. 13. The plot reveals a marked shift in frequency of transmitted wave to a lower frequency. This frequency shift can be used to design various amplitude-dependent wave manipulation devices.

It is also interesting to note from the frequency spectrum of Figure 2. 13 that there is a lower frequency $(\Omega=1.6)$ component present in this case while the component at the same frequency as the excitation is attenuated. This frequency will be used consequently as the basis for design of a Direction-Biased Waveguide (DBWG) test-article.

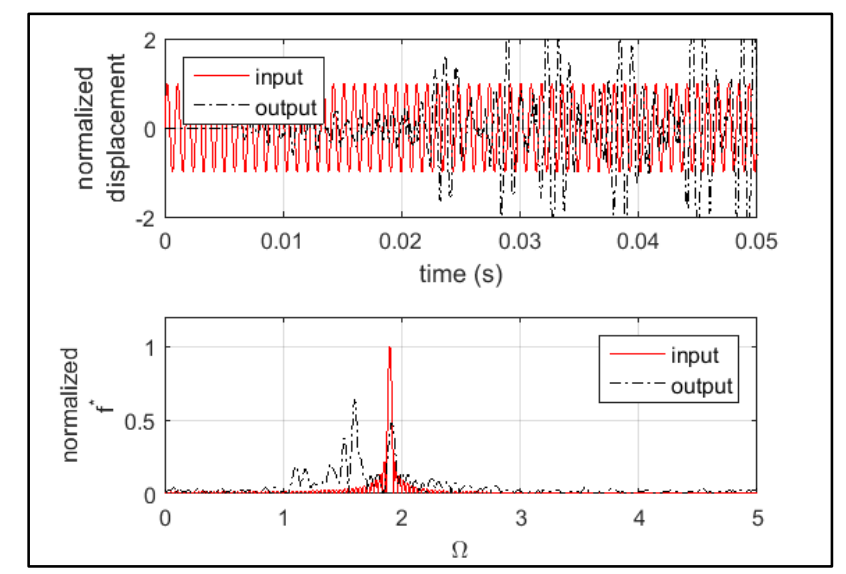

Figure 2. 13: Shift in the frequency of transmitted wave by the NLAM for an incident wave traveling left to right at high amplitude. 


\subsubsection{Direction-Bias Waveguide (NLAM + LRAM)}

Based on the simulation results showing a shift in frequency, an acoustic diode-like device can be conceived. This is done by exploiting the amplitude-dependent propagation and modification of frequency spectrum. Depending on the behavior exhibited by the NLAM chain, an LRAM is suitably selected in order to obtain the direction-bias effect through a lattice chain.

A simulation model is set up in Abaqus with 1000 unit cells. This model is a representation of a long waveguide with a test-article inserted at the center, dividing the waveguide into two equal lengths. Each section of the waveguide was assigned with 400 unit cells. The test-article consisted of two parts - NLAM and LRAM, each consisting of 100 unit cells. The first mass of the waveguide is chosen for application of displacement and this was treated as the 'input'. Displacement response 5 unit cells after the test-article is recorded and is treated as 'output'. Both time history and frequency spectrum of the input and transmitted (output) displacements are recorded for post-processing.

The stiffness curves for the selected NLH are shown in Figure 3. 13. On setting $k_{n}=0$, the linear stiffness of the nonlinear spring is retained and the frequency corresponding this stiffness is 600 Hz. This occurs at small displacements as can be deduced from Equation 2.1 when setting the displacement, $x$ to a very small value. The nonlinearity of the NLH oscillator is triggered only after an appreciable displacement is attained by the attachment mass, thus making the oscillator's response quasi-linear at small amplitude displacements. It is worthwhile to note here that consistent units (SI) are used throughout the simulations. A schematic of the simulation setup in Abaqus is illustrated in Figure 2. 14 (a) and the representative mass-spring representation is shown in Figure 2. 14 (b). 


\begin{tabular}{|c|c|c|c|}
\hline Waveguide & NLAM & LRAM & Waveguide \\
\hline 400 & 100 & 100 & 400 \\
& & & Right to Left \\
& & & Input \\
\hline
\end{tabular}

(a)

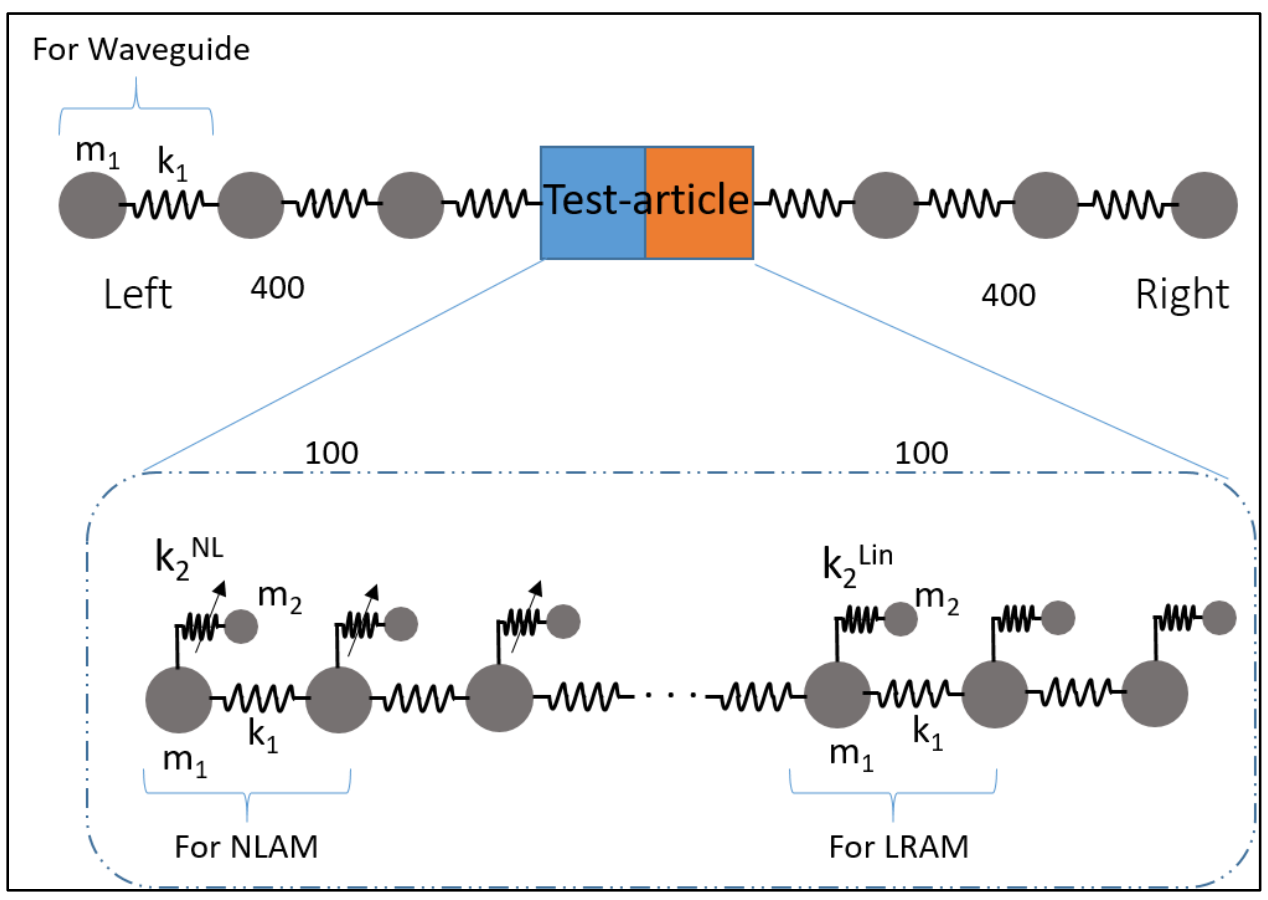

(b)

Figure 2. 14: (a) Schematic of the simulation model in Abaqus and (b) mass-spring arrangement used for testing direction-bias.

The LRAM is designed such that the system exploits the behavior of NLAM such that it is capable of filtering the frequency of excitation and allows $\Omega=1.6$ to propagate. This is done by shunting the two lattice chains together. The following parameters were selected for the LRAM.

$$
\left[m_{1}, m_{2}, k_{1}, k_{2}, k_{n}\right]=[1.66 E-3,0.45 E-3,2.48 E 6,2.27 E 4,0]
$$


By considering the quasi-linear resonance frequency of the NLAM as normalizing parameter, the respective band gaps of NLAM and LRAM are as follows

$$
\begin{gathered}
\operatorname{NLAM}\left[\Omega_{L}-\Omega_{U}\right]=[1-1.6] \\
\operatorname{LRAM}\left[\Omega_{L}-\Omega_{U}\right]=[1.86-2.1]
\end{gathered}
$$

With the selected LRAM parameters, a querying frequency of $\Omega=1.9$ was applied at two different amplitudes along two directions respectively. In other words, four cases are simulated depending on the amplitude and direction of traverse of the wave.

1. Low amplitude, moving left to right (NLAM first).

2. High amplitude, moving left to right.

3. Low amplitude, moving right to left (LRAM first)

4. High amplitude, moving right to left

The output and input response of each of these cases is plotted in Figure 2. 15, which shows a clear indication of direction-bias effect. At high amplitude, a wave incident first on the NLAM side experiences relative propagation. While in the opposite direction the wave is attenuated. Moreover, at low amplitude, the same wave is attenuated irrespective of its direction of traverse. The mechanism of direction-bias is explained in the following section.

At low amplitude $(\mathrm{A}=1 \mathrm{E}-5)$ excitation at the queried frequency, a wave moving from left to right meets the NLAM first, for which the frequency lies outside the band gap; causing the wave to propagate through it. As the frequency lies within the band gap of LRAM, the wave is attenuated by it and hence the wave undergoes an overall attenuation, across the device. 


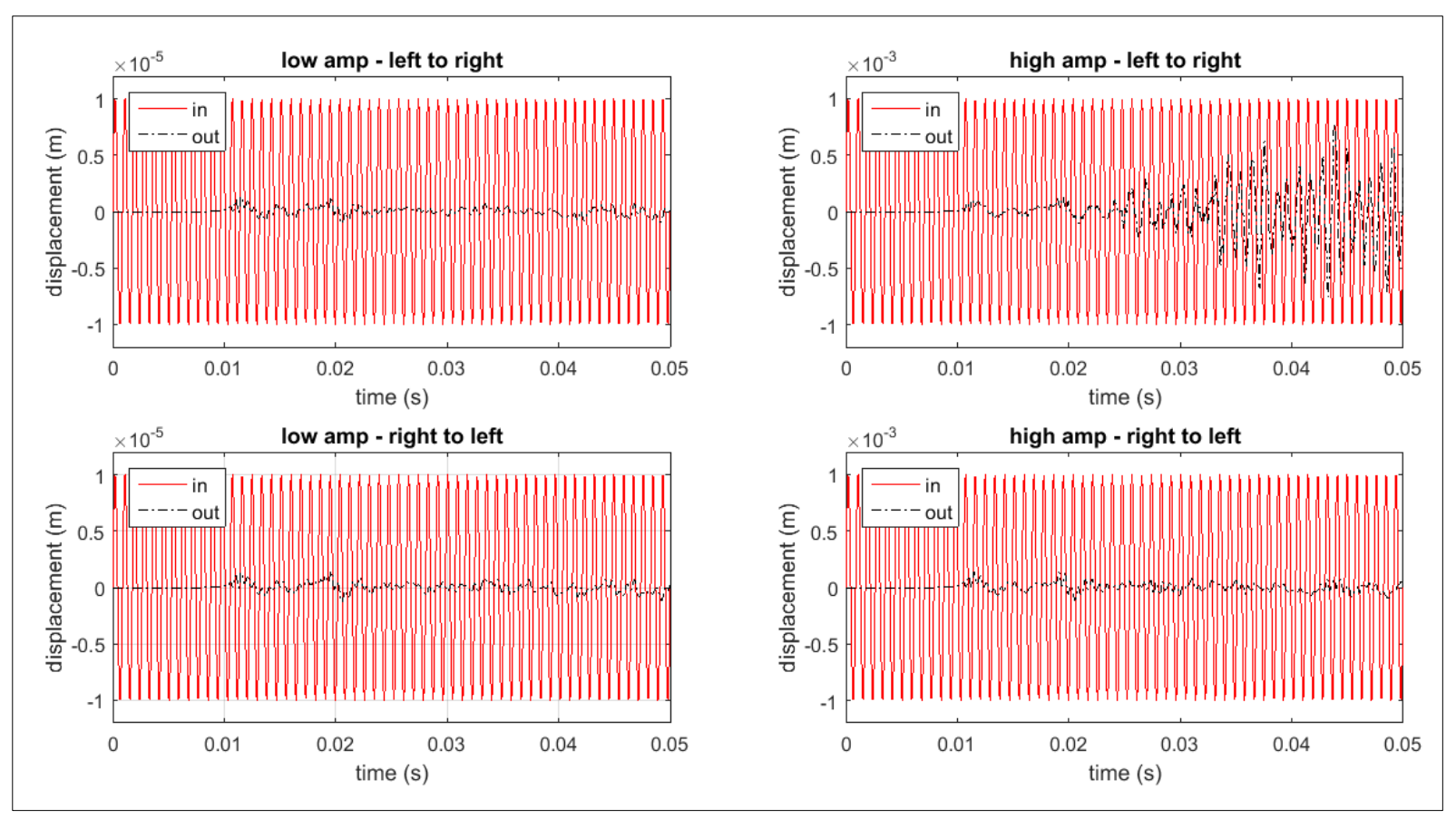

Figure 2. 15: Direction-bias effect obtained in the designed DBWG from simulations.

The same wave, at the same amplitude but traveling in opposite direction is now considered. As it encounters LRAM first, it is attenuated as it progresses along the waveguide. Moving right to left at high amplitude, the wave experiences an overall attenuation similar to the previous case.

On applying excitation to propagate from left to right, the same wave at high amplitude is now observed. At this excitation frequency, it was shown that the wave experiences a partial attenuation at the same frequency but the propagated wave also demonstrates a shift to lower frequency $(\Omega \sim 1.6)$. This propagated frequency lies clearly out of the band gap of the LRAM and hence is not attenuated by it. Thus, in this case, the wave propagates through the waveguide. As can be seen from Figure 2. 15 that although the amplitude of transmitted wave is small in comparison to that of the incident wave, the phenomenon is evident when the relative attenuations for each case are considered together. 
Table 2. 1 shows the characteristics of the proposed direction-biased waveguide device, where 'yes' and 'no' are indicative of relative propagation behavior.

Table 2. 1: Propagation characteristics of the proposed direction-biased waveguide design

\begin{tabular}{|c|c|c|}
\hline Configuration & Amplitude (at $\Omega=1.9)$ & Propagation \\
\hline \multirow{2}{*}{ Left to Right } & Low & No \\
\cline { 2 - 3 } (NLAM first) & High & Yes \\
\hline Right to Left & Low & No \\
\cline { 2 - 3 } (LRAM first) & High & \\
\hline
\end{tabular}

The simulation studies in this section were performed keeping experimental viability in view. The stiffness and mass values are chosen to be manufacturable at the scale of an indoor lab environment.

\section{$2.4 \quad$ Device Implications}

The dispersion characteristics captured through perturbation techniques demonstrate modification in the response of locally nonlinear AM. This results in rich dynamic behavior such as amplitudedependent propagation of waves.

LRAM has the ability to filter waves of the specific frequency band. By adding nonlinearity, amplitude-dependence is introduced. This enriched behavior can be exploited to engineer devices that allow amplitude-dependent selective filtering or act as acoustic switches. This helps to go beyond resonance and explore applications that cannot be displayed by LRAM which is limited by the linear response.

For instance, the system with parameters given in can act as a filter for low amplitude wave at $606 \mathrm{~Hz}$. However, the device allows the wave at the same frequency to propagate at a higher amplitude. A strategically chosen combination of NLAM and LRAM can be used to obtain 
direction-bias in wave propagation. This system can result in engineering an acoustic diode by acting as a one-way insulator. Also, employing such unidirectional wave propagating devices can find applications in areas demanding vibration isolation or reflection free media.

By tuning these devices, it can be possible to shield an object from a specific range of querying frequencies and hence aid in avoiding detection. This results in acoustic cloaking. A pure nonreflective boundary inherently carries much value as it possesses the ability to act as a sink, which can find applications in experimental investigations.

\subsection{Summary}

The objective of this section is to introduce and study the effect of including nonlinear stiffness in the local attachment of an otherwise LRAM system. This was achieved by studying the response

of the cubic hardening type Duffing oscillator. The consequence of adding nonlinear stiffness in place of a linear system can result in complex behavior. This was validated through numerical investigation of Duffing oscillator's response to the frequency of excitation and the direction of changing frequency. Lindstedt-Poincare perturbation approach was applied to a few 1-D massspring systems to derive the dispersion behavior and postulate devices that may exploit the behavior indicated by the dispersion curves thus obtained. Numerical studies on NLAM chain exhibited interesting amplitude-dependent propagation phenomenon which was used to postulate a direction-bias waveguiding device. It was designed by employing an LRAM with the NLAM. A numerical simulation conducted on the DBWG design gave an indicative evidence of directionbias that will be used as the basis of experimental studies in the subsequent section. 


\section{CHAPTER III}

\section{EXPERIMENTAL VERIFICATION}

\subsection{Introduction}

The simulation studies carried out in the previous chapter revealed varied amplitude-dependent phenomenon in wave propagation by the introduction of local nonlinearity. Of the several possible device implications, one was the acoustic diode. The evidence of direction-bias demonstrated through simulations motivated the attempts to investigate this behavior through experimental methods.

An experimental rig similar to simulation model is constructed to enable investigations of the direction-bias phenomenon. An account of the test setup is presented describing the various components involved. The following sections present the process involved in design, manufacture, and characterization of test-articles and the waveguide bed. Experimental methodology is described and results obtained are analyzed. A contact-based approach is used to realize the desirable nonlinear stiffness in the local attachment. This method is described in detail and the respective program is provided in the appendix.

\subsection{Experimental Setup and Methods}

The objective of the experiment is to investigate the direction-bias effect across a specifically designed test-article. The experiment involves measurement of response across the test-article when an excitation is applied on one end. To test the direction bias, two different amplitudes and directions of excitation are required as per the mechanism detailed in Section 2.3.3. To enable this type of testing, an experimental rig was designed. Figure 3. 1 shows a schematic outline of the 
experimental set up which enables propagation of waves along a waveguide. The waveguide is instrumented with transducers for measurement of response. The far end of the waveguide was tapered to minimize the effect of reflections. This oblique end is embedded in a sand-bed to ensure minimization of reflections from a free boundary. Suspension strings were used to provide periodic supports along the length to the rather slender waveguide assembly. Lateral line-support arrangement is made before and after the test-article to mitigate any mode conversion to transverse mode of the beam.

The components of the system are illustrated in the Figure 3. 2 which shows the (a) shaker assembled with the waveguide with the test-article assembled at the location (e) at the interface of two sections similar to $(\mathrm{g})$. The amplifier is located at (c) which receives the signals from the waveform generator (b). The lateral (f) and vertical suspension (d) supports are employed to act as a guide to the polycarbonate beam against twisting under self-weight. The vertical supports are equipped with special lead screw hooks for a fine leveling of the beam. Leveling of the 35 feet long beam was achieved with the help of laser level markers and bubble indicators. The location of the transverse supports and suspension points enable flexibility to mitigate interruptions from the bending modes of the beam.

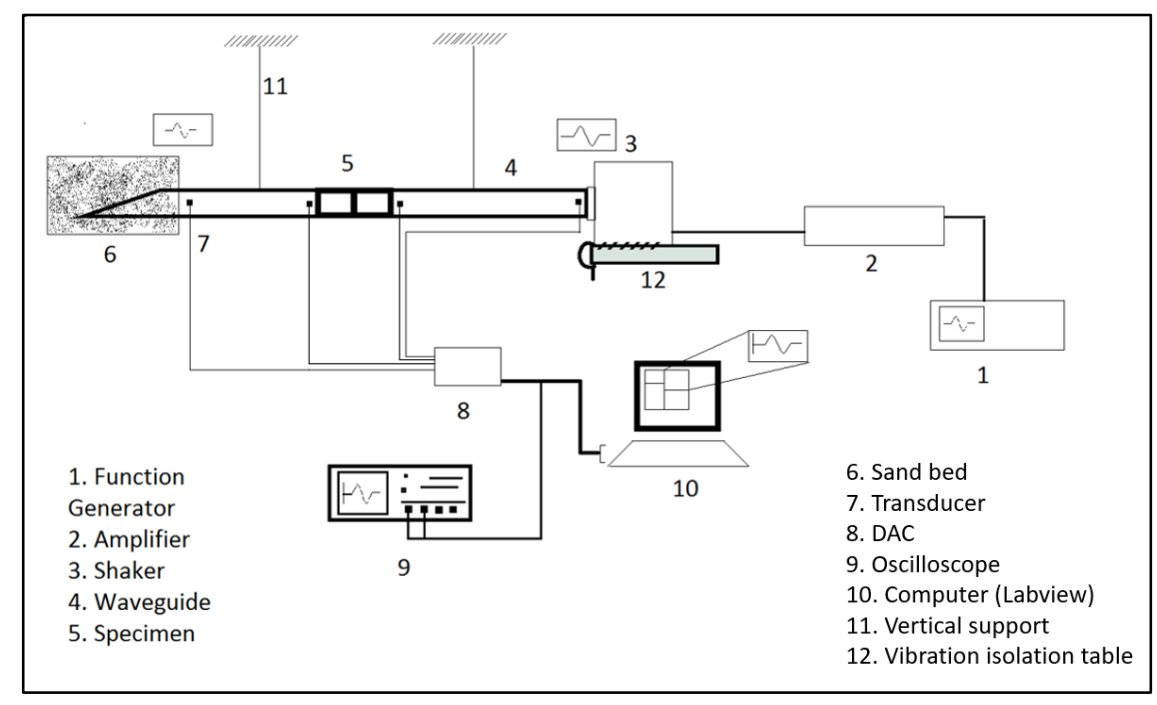

Figure 3. 1: Schematic of the experimental setup. 


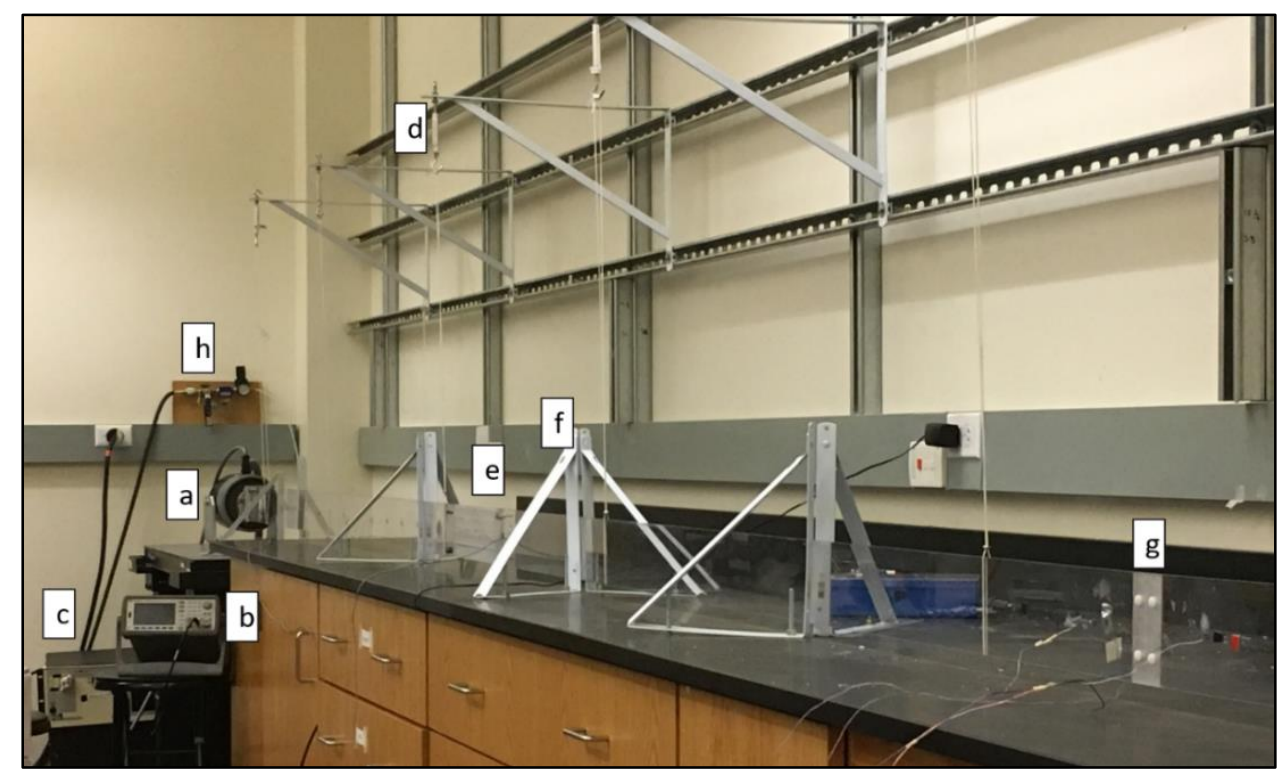

Figure 3. 2: Assembled experimental set up showing various components of the system.

A Labworks ET-140 shaker, which is a moving armature type $110 \mathrm{lbf}$ capacity electrodynamic shaker is used for actuation. The trunnion mounting base allows for positioning of shaker at different angles. The experimental set up demands horizontal orientation of shaker, because of the principle direction of waveguide assembly. The Keysight waveform generator is used to synthesize driving signals for shaker which are pre-amplified by Labworks pa-141 power amplifier before they are fed into it. The LW pa-141 is equipped with a gain control dial that enables up to $36 \mathrm{~dB}$ voltage gain. A gain setting of $20 \%$ is employed consistently in all of the experiments mentioned in this study. Single axis B\&K accelerometers (4507 and 4508) are used to record the response of the points at locations mentioned earlier. Single frequency excitations are applied to make translation from acceleration provided by accelerometer to displacement straightforward since harmonic displacement and acceleration are related as

$$
u=-\frac{\ddot{u}}{\omega^{2}}
$$

Where $u$ is displacement and $\omega$ is the frequency of harmonic motion. These accelerometers have an input voltage of $5 \mathrm{~V}$ and a sensitivity of $10 \mathrm{mV} / \mathrm{ms}^{-2}(98.9 \mathrm{mV} / \mathrm{g})$. 
The NI USB 6009 DAC in conjunction with NI LabVIEW was employed for data acquisitions in the experiments in this study. LabVIEW was the primary data collection platform for all accelerometer measurements throughout. A sampling rate of $24 \mathrm{kSa} / \mathrm{s}$ collecting $24 \mathrm{k}$ samples ensured a window of $1 \mathrm{~s}$ on the LabVIEW GUI. The GUI developed as part of investigations (Figure 3. 3) allows for migration of data with the flick of a toggle switch to excel for analysis of data. Acceleration time histories exported to excel files are processed using Matlab to obtain the frequency content to carry out analysis. In certain conditions, non-contact measurements are made with the Polytec PDV-100 Laser Vibrometer which, in conjugation with the 'Polytec Vibsoft' software enables us to export time history of the velocity of a point. The Vibrometer works on laser triangulation principle and requires a reflective surface to make a clean measurement which is ensured by using a reflective tape mounted on the surface in context. To obtain velocity history of the cantilevered tip mass, for instance, a small portion $\left(5 \mathrm{~mm}^{2}\right)$ is covered with reflective tape and the laser is shone on this area. Measurement of this type would otherwise have been not possible without interfering with the queried system. Velocity response was exported to excel and post-processed using MATLAB.

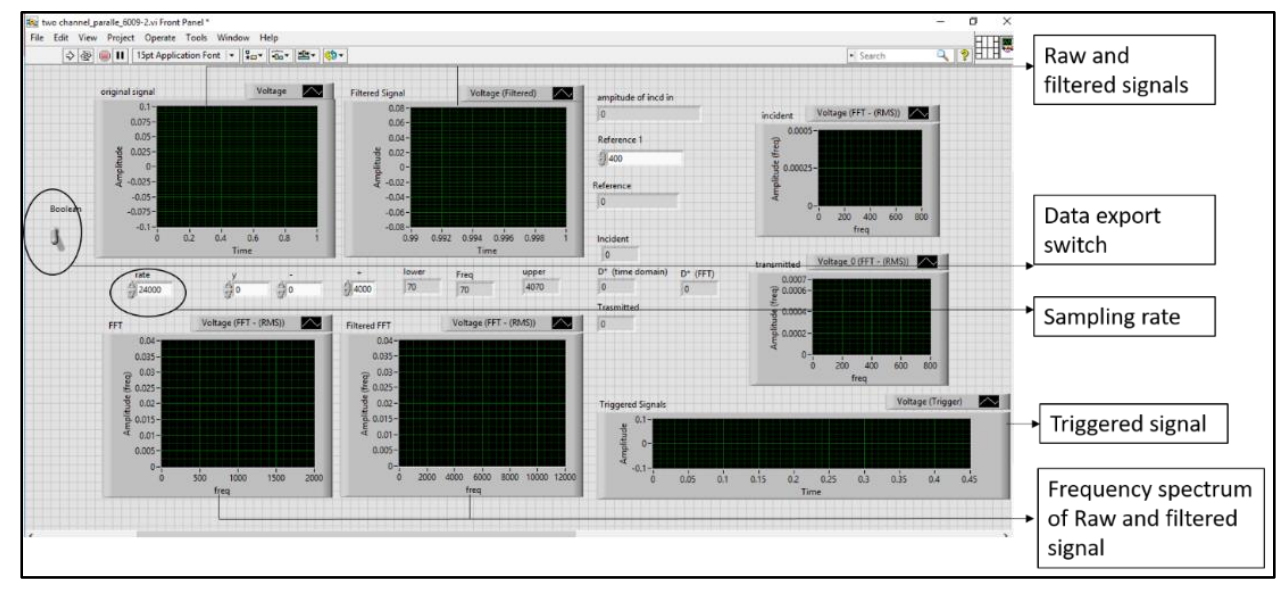

Figure 3. 3: LabVIEW interface developed for signal extraction. 


\subsubsection{Waveguide Assembly and Characterization}

The waveguide assembly is built with $1 / 4^{\prime \prime}$ thick $8^{\prime} \times 6^{\prime}$ raw polycarbonate plate cut into equal sections of 4" width, each 8' long. Sections are provided with interface connection features for assembly using bolts. Assembled length of the finished waveguide assembly is 35 feet and it is suspended using nylon strings to keep it leveled. The first section is provided with a specific set of holes for interfacing it with the forcing device (shaker). The fixture used for transmission of force from shaker to the waveguide is shown in Figure 3. 4 with SolidWorks model and assembled setup. The accelerometer is mounted (c) on the shaker head as shown in the figure.

The figure also illustrates the waveguide (d) attached to the shaker (a) via the fixture (b).

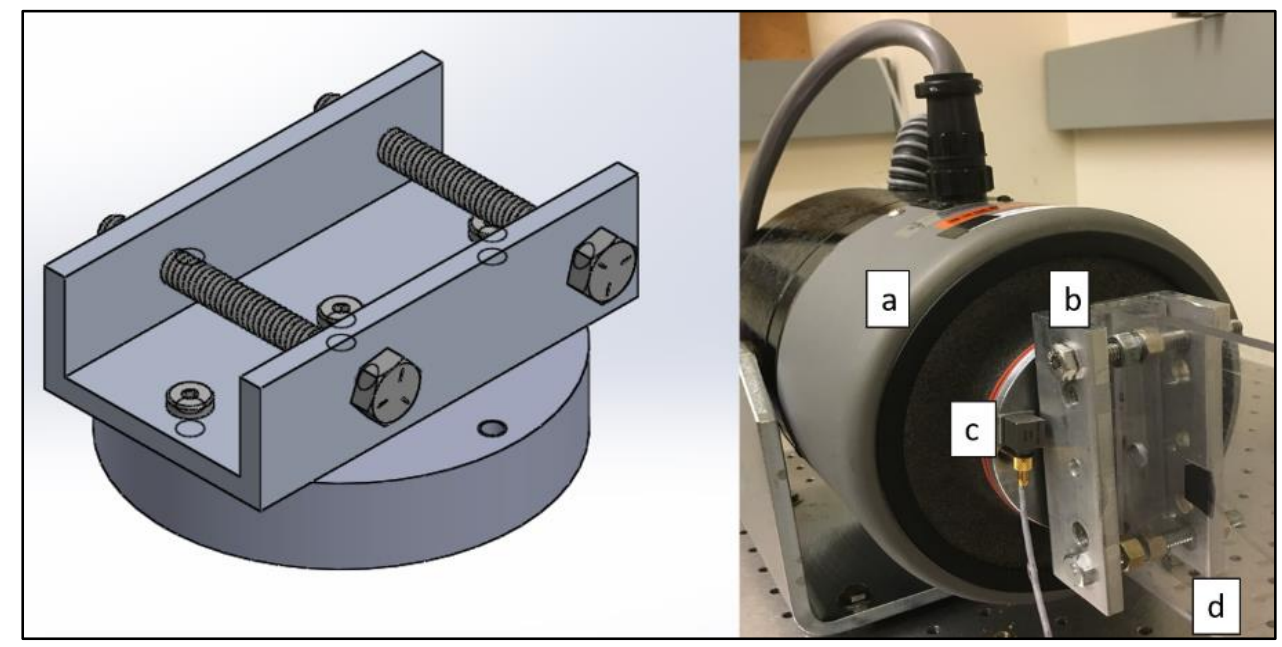

Figure 3. 4: CAD model and assembled fixture for transmission of force from shaker to the waveguide.

Initial waveguide characterization experiments were performed, which involved correlation of bar-velocity, wavelength and effect of the sand boundary on reflections. The objective of this experiment was to experimentally determine velocity of an elastic longitudinal wave [65] in the waveguide and compare it against analytical value which is given by 


$$
c=\sqrt{\frac{E}{\rho}}
$$

To experimentally obtain the wave speed, the time taken by a wave to traverse two points separated by a known distance is recorded. To do so, two accelerometers were placed 16 feet $(4.88 \mathrm{~m})$ apart on the waveguide with their principal direction of measurement along the longitudinal direction. A $1 \mathrm{kHz}$ 'single-cycle-sine-burst' waveform is deployed by shaker and the time delay in the approach of the wave at respective transducers is recorded. Calculation of velocity is hence a straightforward task at this point. The experimentally obtained velocity of $1400 \mathrm{~ms}^{-1}$ is found to be in good correlation with that obtained theoretically as $1350 \mathrm{~ms}^{-1}$ with a difference of $\sim 3.5 \%$ being attributed to error from choosing exact times of arrival of the wave at transducers. Also, theoretically assumed density and elastic modulus are seldom exactly the same as in a physical system, giving rise to such discrepancy. However, small deviation as $3.5 \%$ can safely be disregarded as being within the general engineering tolerance of $5 \%$. Figure 3.5 illustrates the arriving of signals in time while the mechanical properties of Polycarbonate per manufacturer test-article are enlisted in Table 3.1.

\section{Table 3. 1: Mechanical properties of Polycarbonate}

\begin{tabular}{|l|l|}
\hline Property & Value \\
\hline Elastic Modulus $\left(\mathrm{kg} / \mathrm{m}^{3}\right)$ & $2.2 \mathrm{E} 9$ \\
\hline Density $\left(\mathrm{kg} / \mathrm{m}^{3}\right)$ & 1200 \\
\hline Bar Velocity $(\mathrm{m} / \mathrm{s})$ & $1.35 \mathrm{E}^{\#}$ \\
\hline
\end{tabular}

${ }^{\#}$ note that bar velocity is the theoretically calculated using material properties per Equation 3.2 


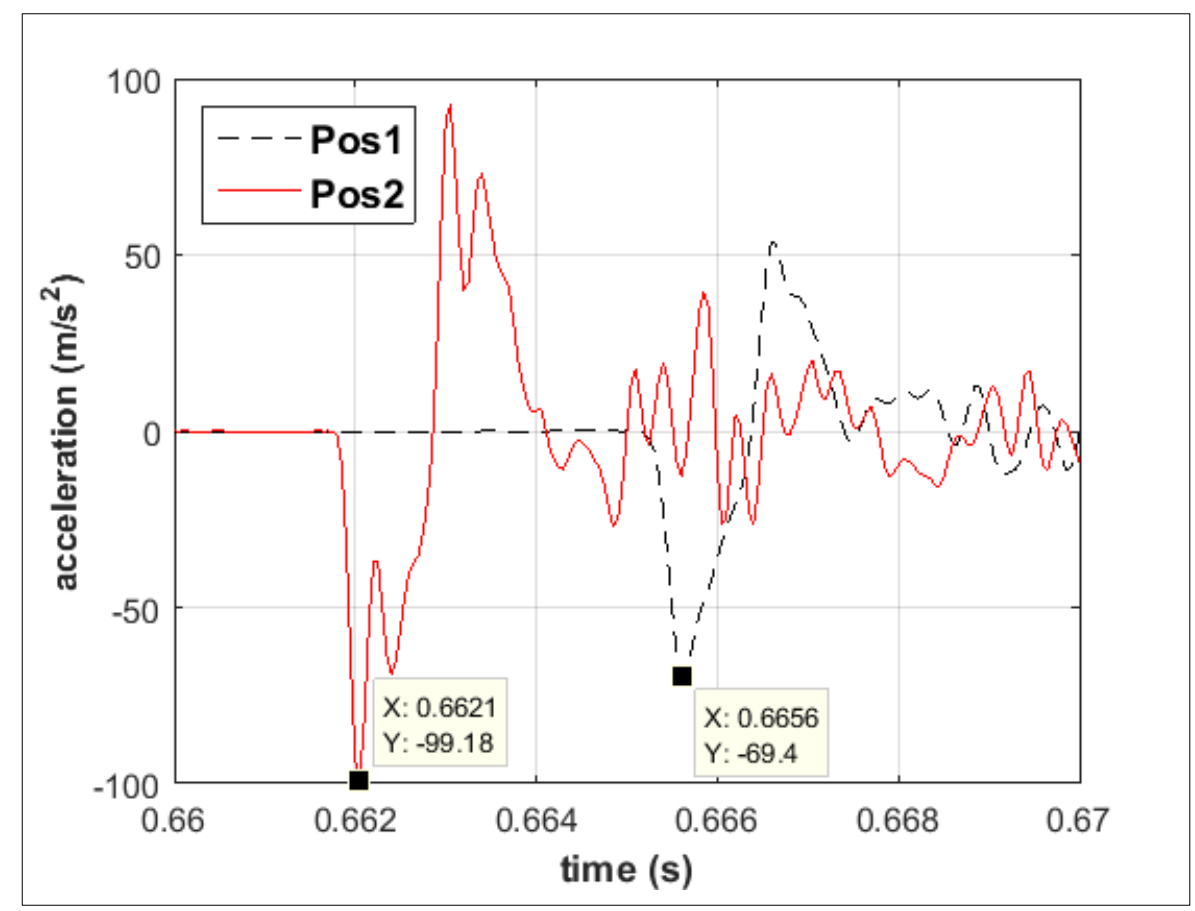

Figure 3. 5: Measurement of wave velocity for the waveguide assembly.

For an applied excitation, the wavelength of waveguide must follow the equation given by the general dispersion relation for a continuous beam, $\lambda=\frac{\omega}{c}$

To check the wavelength of the longitudinal wave, accelerometer readings were taken from points 1" apart along the length of the beam when harmonic single frequency excitation was applied at one end of the waveguide. The acceleration history was recorded and displacement of each point at a given time instant was plotted against the position of the point along the waveguide. The wavelength obtained at the applied $1 \mathrm{kHz}$ can be visually determined as seen in Figure 3. 6. This value of $1.376 \mathrm{~m}$ was found to be in good agreement with the theoretically determined wavelength of $1.35 \mathrm{~m}$ obtained from the known wave velocity and frequency of the wave. 


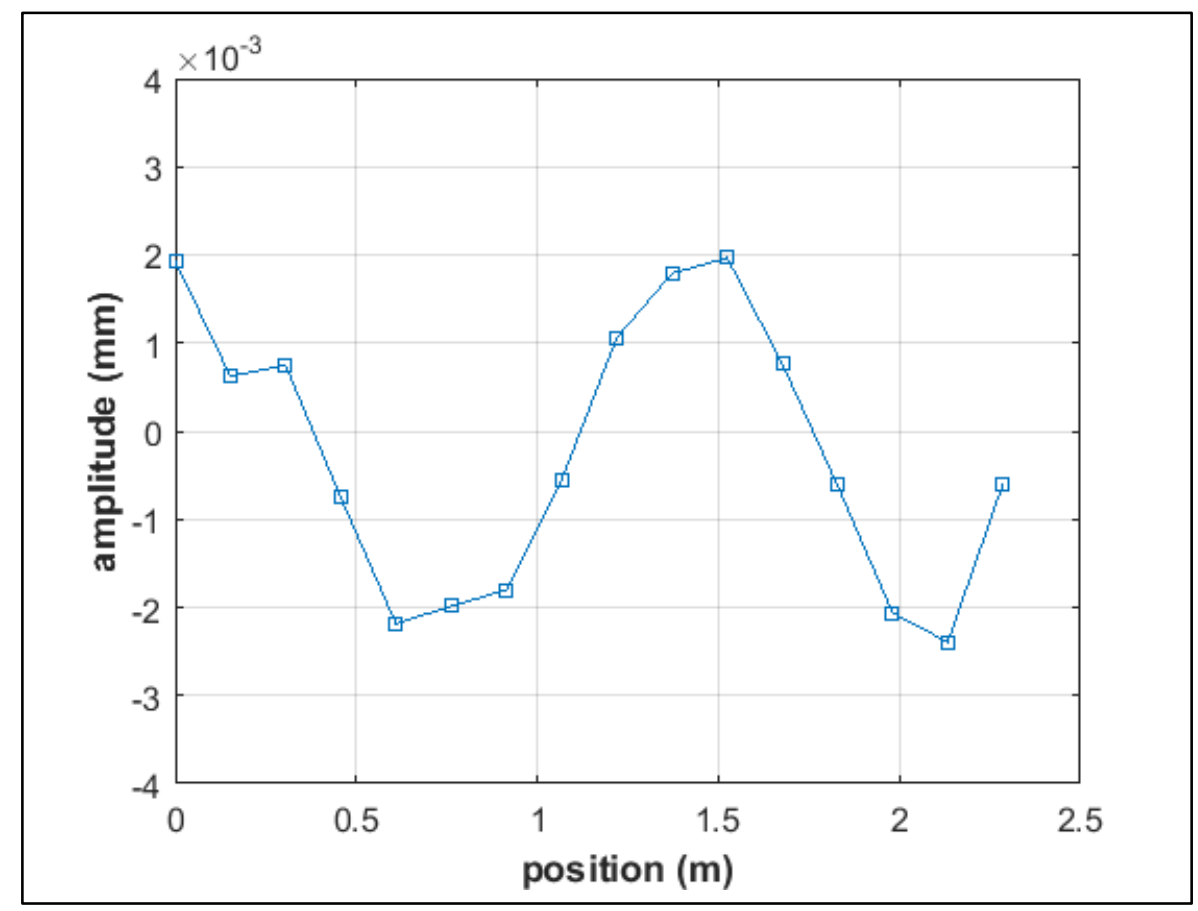

Figure 3. 6: Snapshot of displacement history of points separated along the length of the waveguide for a $1 \mathrm{kHz}$ continuous sine excitation.

Sand boundary enforced on the far side of the waveguide was designed to provide a reflection free boundary conditions to neglect any interference with reflections. However, unlike in the case of Smith et al [17] where the interacting waves were flexural and waveguide was an aluminum beam of cross-section $6.35 \mathrm{~mm} \times 12.7 \mathrm{~mm}$, the case at hand is that of a longitudinal elastic wave traveling in a $6.35 \mathrm{~mm} \times 101.1 \mathrm{~mm}$ polycarbonate plate. This reduction in the area of contact could be a cause for the ineffectiveness of this setting for the designed purpose. It, however, provides a free type boundary condition providing support in the vertical direction for an otherwise freely hanging thin beam highly susceptible to torsion due to its own weight. Figure 3 . 7 shows the time history recorded with an accelerometer placed at $4.5 \mathrm{~m}$ from shaker head for a single cycle $1 \mathrm{kHz}$ sine excitation moving towards the boundary. The response from the sand boundary is compared with that of a fixed type boundary fabricated specifically for this test condition, shown in the figure. 


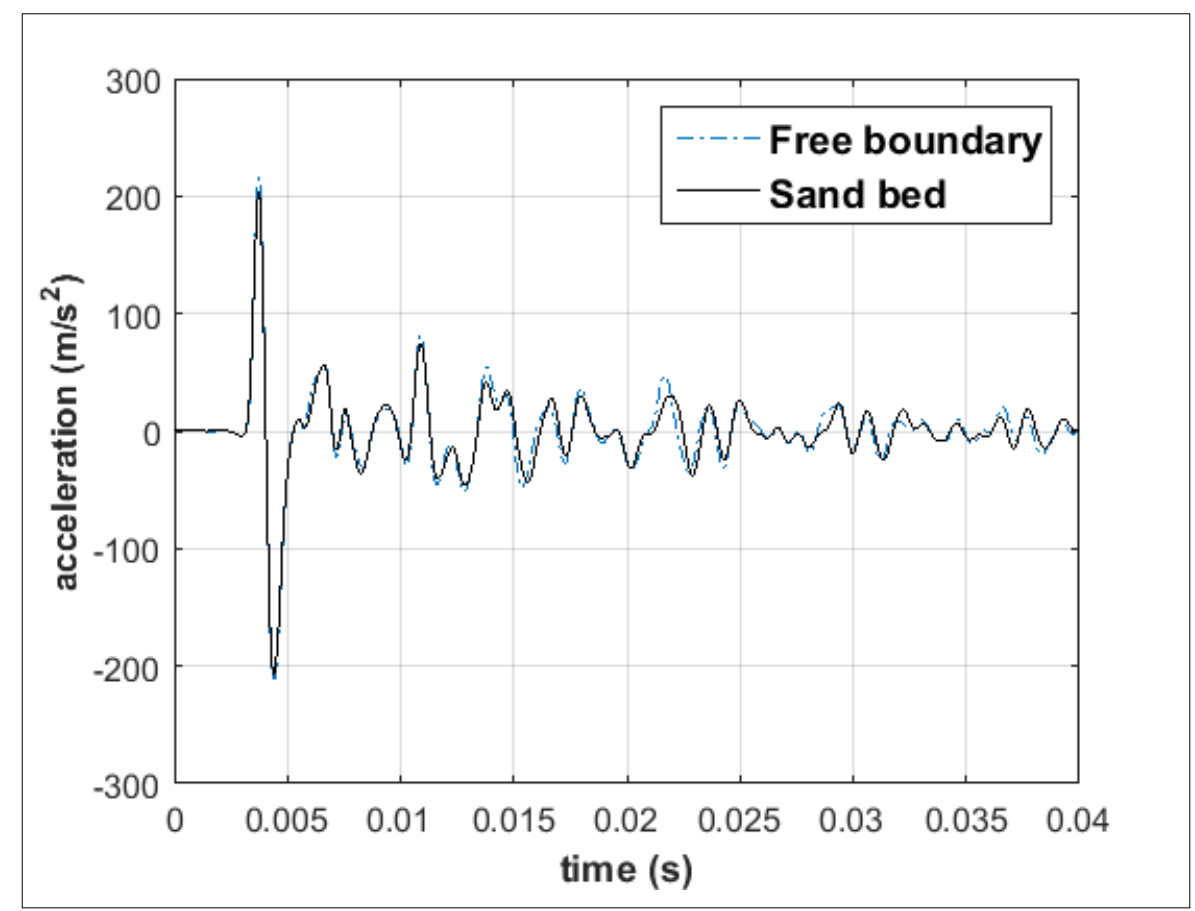

Figure 3. 7: Characterizing the behavior of employed boundary condition by comparing the acceleration response at a point on the waveguide.

The focus of this part of the study is to experimentally investigate propagation behavior through a test-article designed to act as the mentioned Direction-Bias Waveguide (DBWG). With parameters selected based on the previously demonstrated numerical study, an LRAM and its nonlinear counterpart NLAM were manufactured out of polycarbonate and assembled with a 35 feet long waveguide beam. Steady state longitudinal displacements along forcing direction are used to obtain amplitude ratios $\left(D^{*}\right)$ at different frequencies of excitation to analyze transmission behavior across the test-article.

A $1 / 4$ " thick polycarbonate plate with 4 " width is used consistently for all parts (LRAM, NLAM, and waveguide) included in the experiment to keep assembly consistent and simple. One of the most important features of the test-article in context was local resonance phenomenon. This was done by removing mass from a polycarbonate plate, resulting in cantilever inclusions along the length. The dimensions of inclusion cantilevers are controlled in order to tune their resonance 
frequency. Traditional thin beam theory was used to theoretically calculate natural frequency of the cantilever with tip mass to match with simulation parameters. To keep simulation results valid for experimental viability, both simulation and experimental parameters were planned simultaneously.

Ensuring symmetry about the neutral axis and simultaneously keeping all metamaterial features in subwavelength range resulted in a design with four cantilevers along the width of test-article hence, each unit cell was chosen to be 1 " square with a cantilever inclusion. Also, a symmetric design would help counteract any moments associated with dynamics of the cantilevers. The testarticle, therefore, is divided into four horizontal rows on both NLAM and LRAM parts. Defining unit cell area is perhaps the first major step toward designing a metamaterial of this kind as this locks with it, the defining parameters such as $k_{1}$. The values $k_{2}, m_{2},\left(m_{2} / m_{1}\right)$ and hence the band gap limits are interdependent. Further, $m_{l}$, which has been fixed forms the starting point for these design parameters.

The design of test-article proposed in the earlier section requires at high amplitude, to contain a no-propagation condition for $\Omega=1.9$ for a wave traversing through the NLAM first and must allow $\Omega \sim 1.6$ to pass. Hence LRAM essentially behaves as a filter for the frequency of interest, irrespective of the amplitude of excitation. NLAM is the key to modifying the incident wave frequency due to its nonlinear stiffness. Hence, although both parts of the test-article are concurrently designed, the NLAM primary resonance frequency $\left(\omega_{0}\right)$ is chosen as reference.

Each design was validated by part modeling and modal analysis carried out in SolidWorks. On successful completion of design, the detailed drawings generated from SolidWorks were used for fabrication of the corresponding parts. The following sections describe the design and manufacturing of the test-article. 


\subsection{Test-article Design}

\subsubsection{Translation of stiffness between system parameters and design.}

The two stiffness associated with the design of either test-articles are $\mathrm{k}_{1}$ and $\mathrm{k}_{2}$ being the stiffness of host, and local respectively. The chosen method derives the local stiffness from a cantilever beam. This stiffness $k_{2}$ is obtained from the beam deflection relation as

$$
k=\frac{3 E I}{L^{3}}
$$

where I and L are correspondingly the area moment of inertia (about the neutral axis) of cross section and length of the cantilever beam. The method of finding $\mathrm{k}_{1}$ however is shown in Figure 3. 8. The method consists essentially of dividing the portions around unit cells and treating each of them as stiffness. The stiffness represented as (1) and (2) in the figure are then combined based on their arrangement to obtain the effective stiffness, representative of the host structure. The geometry of the unit cell is altered to result in conformity with simulation parameters used in the previous chapter.

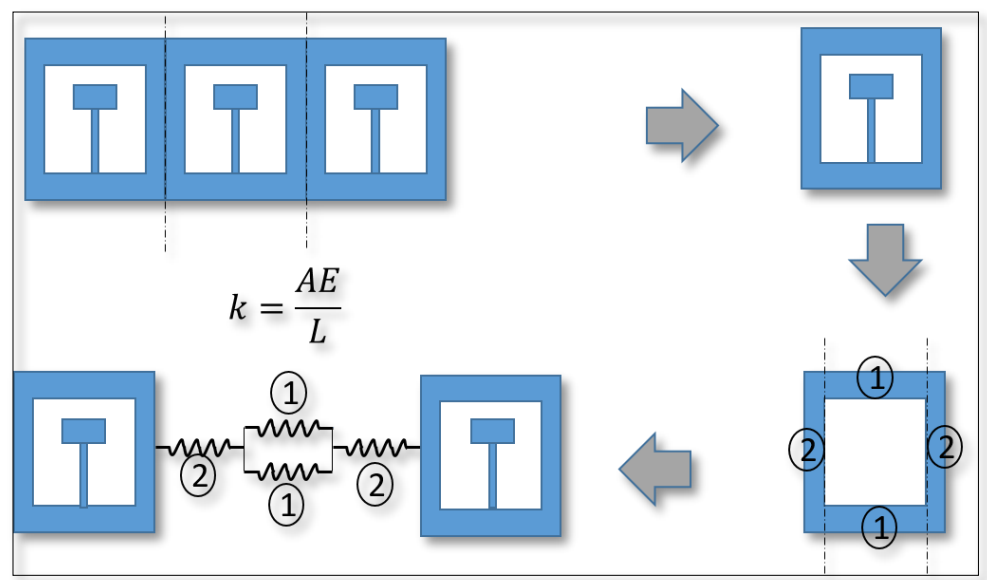

Figure 3. 8: Computing the host stiffness for an LRAM component. 


\subsubsection{Locally Resonant Acoustic Metamaterial (LRAM)}

With parameters derived from simulations, the LRAM part of test-article is designed with the following setting

$$
\left[m_{1}, m_{2}, k_{1}, k_{2}, k_{n}\right]=[1.69 E-3,0.46 E-3,2.48 E 6,2.27 E 4,0]
$$

The method described in the previous section was used to obtain the stiffness, $k_{l}$. With $k_{1}$ determined and unit cell size fixed at 1 " square, the cantilever is designed to accommodate for spatial offsets within a unit cell for oscillation of tip mass. The local resonance frequency measurements were taken by recording the response of each tip mass with a laser Vibrometer and computing frequency content of the time history for an impact type excitation and the obtained spread is shown in Figure 3. 11. The test-article consists of 4 rows and 12 columns of unit cells adding up to a total of 48 unit cells arranged along the length as shown in Figure 3. 10.

LRAM test-article was cut using water jet cutter with no post cutting operation on unit cells as the tip mass was designed to be an integral part of the cantilever. Dimensions pertaining to LRAM test-article are shown in Figure 3. 9. The drawings generated from SolidWorks were exported to AutoCAD in order to generate the g-code for waterjet CNC machine. 


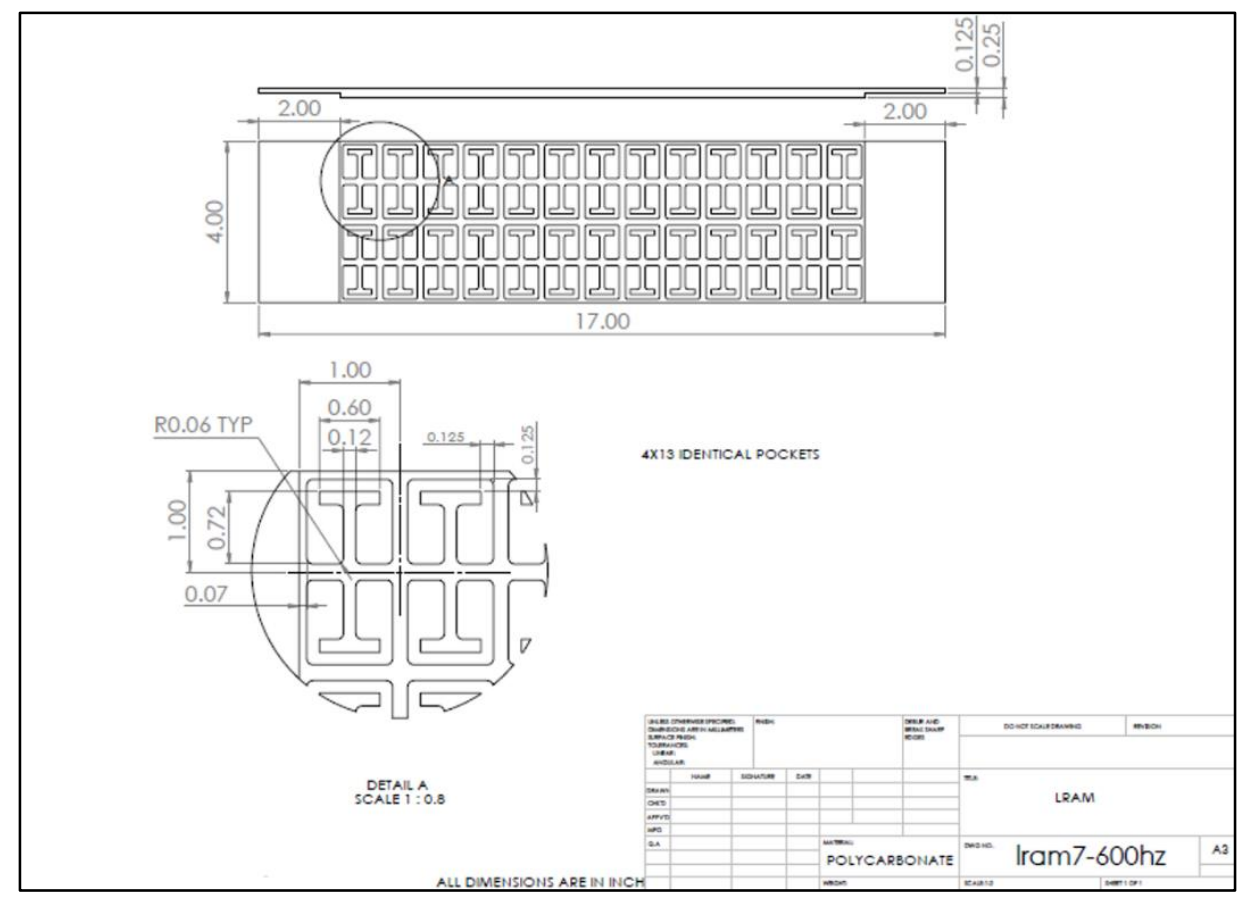

Figure 3. 9: Technical drawing for the LRAM test-article, showing the detailed dimensions.

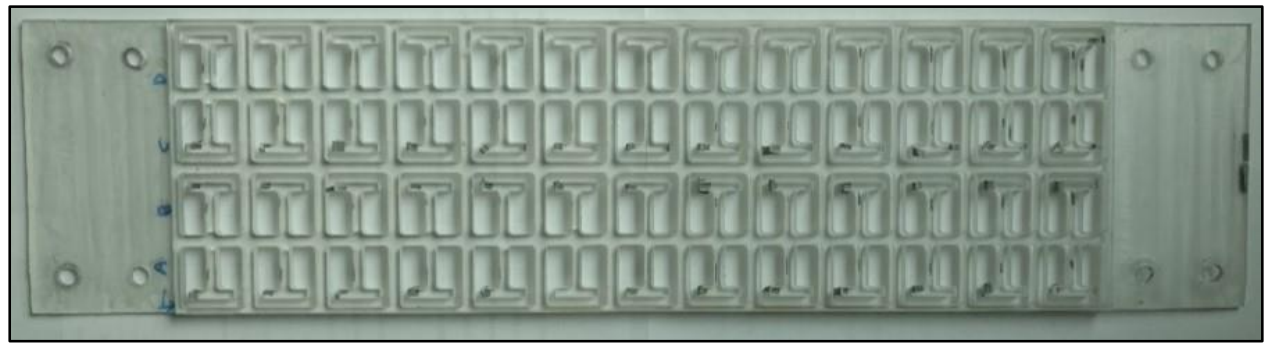

Figure 3. 10: Manufactured LRAM test-article fabricated in a polycarbonate plate. 


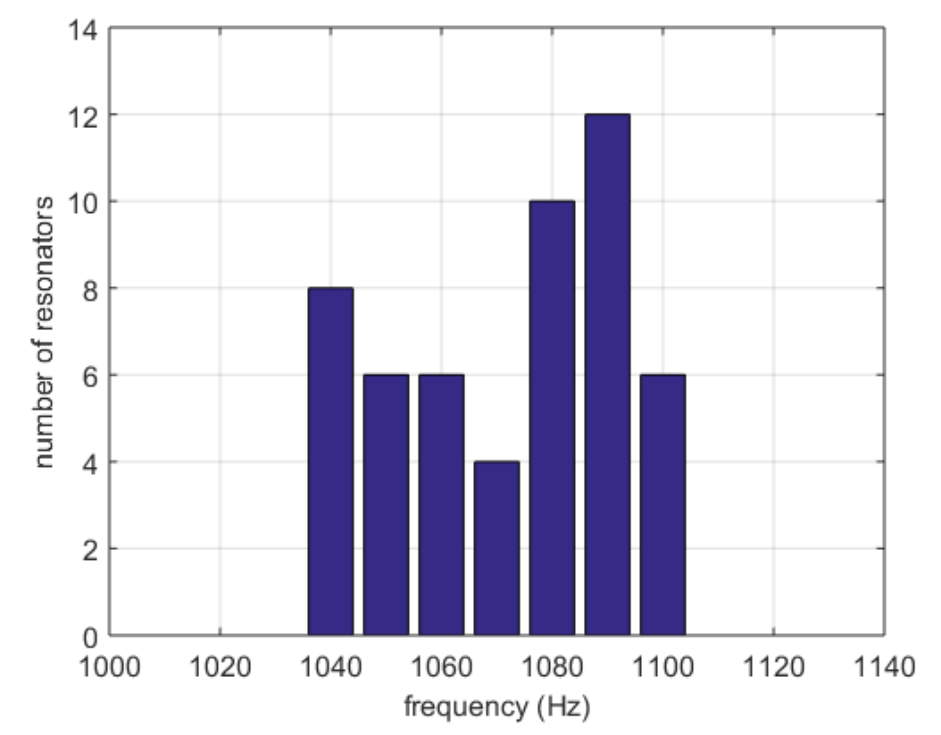

Figure 3. 11: Experimentally recorded local resonance frequencies of LRAM.

\subsubsection{Nonlinear Acoustic Metamaterial}

The current method of realizing specified nonlinearity is based on varying the effective length of cantilever during oscillation [66]. This varying in length is achieved by placing a series of strategically placed rigid support points along the length of the cantilever in the form of a profile. The separation between the point of contact to the tip mass would become the effective length of the cantilever and as the contact point moves further away from the root, (closer to the tip) the effective length decreases. From the Figure 3. 12 (a), it can be seen that this length decreases with increasing amplitude of oscillating mass. During harmonic oscillation of the tip mass and advancing contact with the root profile, the system experiences hardening via this mechanism. The degree of nonlinearity can be varied to obtain a different set of points for the root profile wherein the greatest nonlinearity can be achieved for a flatter and least nonlinearity obtained for a zero radius profile. A schematic is shown in Figure 3. 12 (b) wherein the stiffness curve with and without contacting profile are shown for the same beam. 
Other methods to realize nonlinearity employ magnetic materials, viscoelastic fluids, granular chain contacts and so on. However, the current method was used as it provides a means to do so with a simple structural method. In addition, another advantage of using this system is the ease of designing the required hardening.

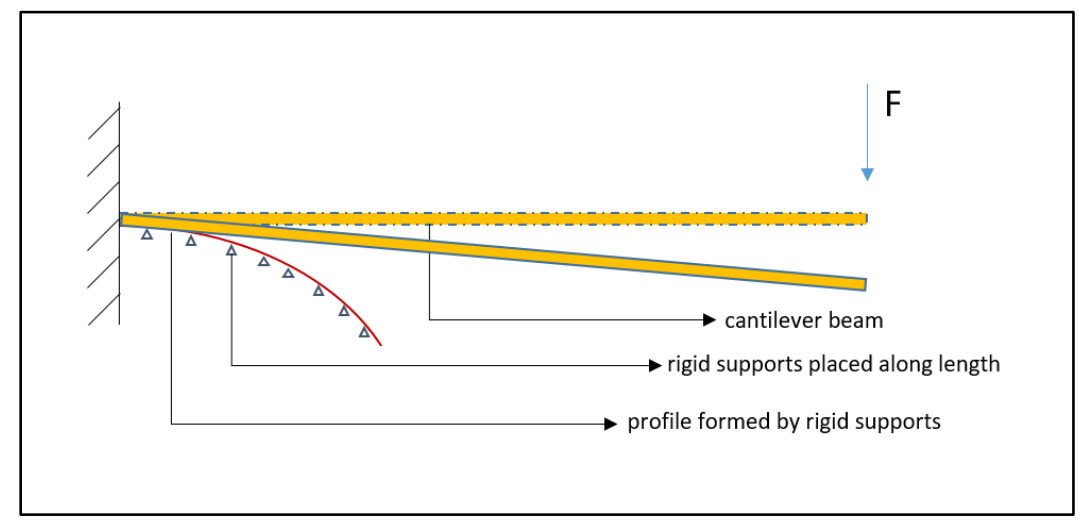

(a)

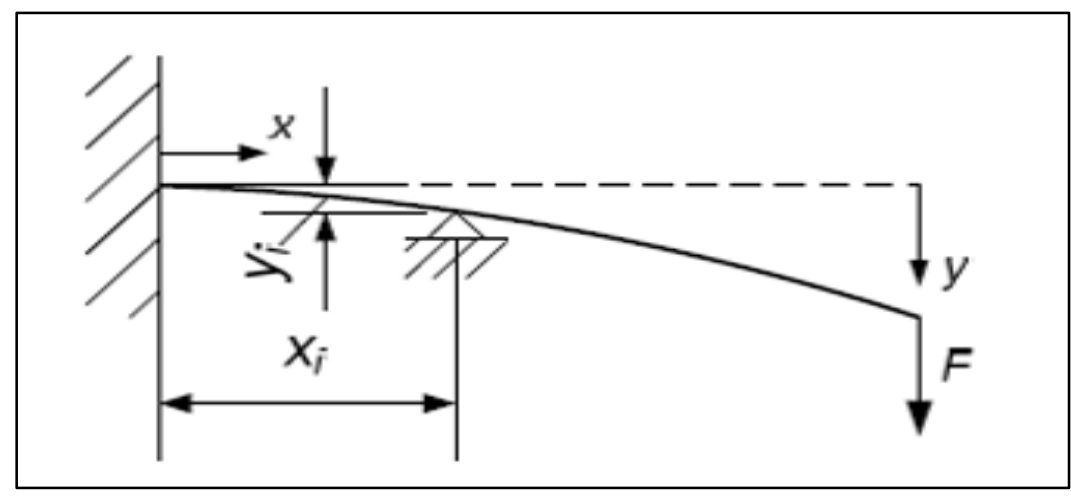

(b)

Figure 3. 12: (a) Nonlinear hardening stiffness obtained through contact with root profile. (b) Method of locating coordinates of the support points using beam deflection theory [66].

Considering manufacturing limitations, the parameters were chosen as shown below.

$$
\left[m_{1}, m_{2}, k_{1}, k_{2}, k_{n}\right]=[1.67 E-3,2.84 E-3,2.43 E 6,40382.45,1 E 11]
$$


As suggested by the simulation parameters of amplitude, a nonlinearity constant of $k_{n}=10^{11}$ was required to obtain the desired direction-bias effect from the test-article. The value of $k_{n}$ is chosen such that at the low amplitude setting the hardening is kept within 5\% of that at high amplitude. In other words, the cantilever has been designed to display linear stiffness at low amplitude and nonlinearity is triggered at the assumed higher bound of excitation amplitude. The method of obtaining the stiffness is described in detail by Spreemann et al [66]. The algorithm to obtain the root profile is shown in Figure 3.14 and the Matlab code used to obtain the root profile for a chosen nonlinear stiffness parameter is provided in APPENDIX-I. Root profile obtained for $k_{n}=10^{11}$ as obtained from the developed code is illustrated in the Figure 3.13 (b), showing its intersection with the beam deflection curve without considering nonlinear effects. In the event where an integral root profile (as opposed to the present assembly) was planned, it was imperative to ensure that intersection of curves was kept into consideration for design. This is because if the minimum diameter of cut offered by the tool were more than $1 \mathrm{~mm}$ (refer to Figure 3.13 (a)), the contact would not be initiated and hence the stiffness would be linear in the region of interest.

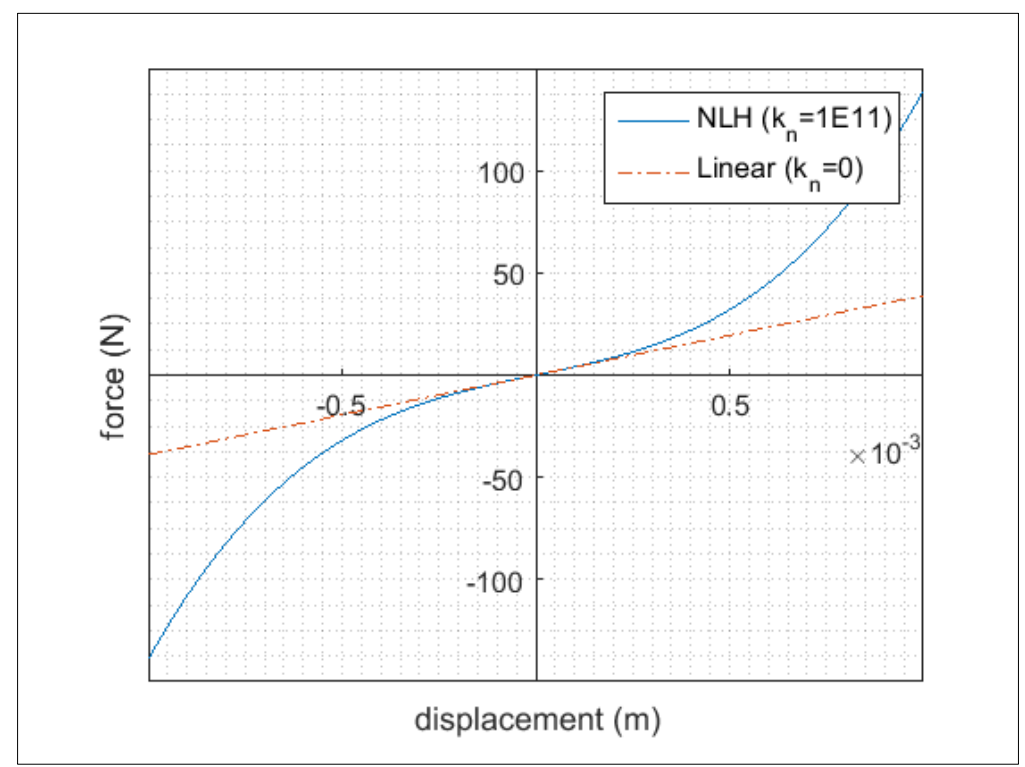

(a) 


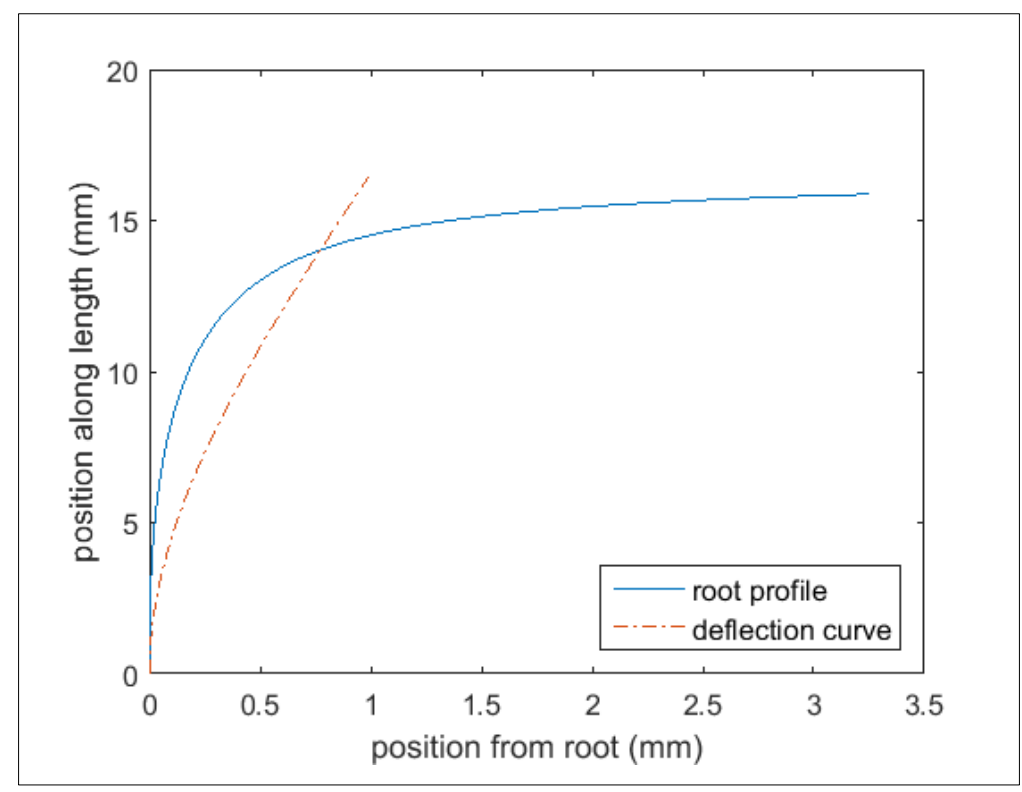

(b)

Figure 3. 13: (a) Input stiffness curve with Linear and NLH force-displacement data and (b) Computed cantilever root profile for obtaining the predefined load-displacement curve.

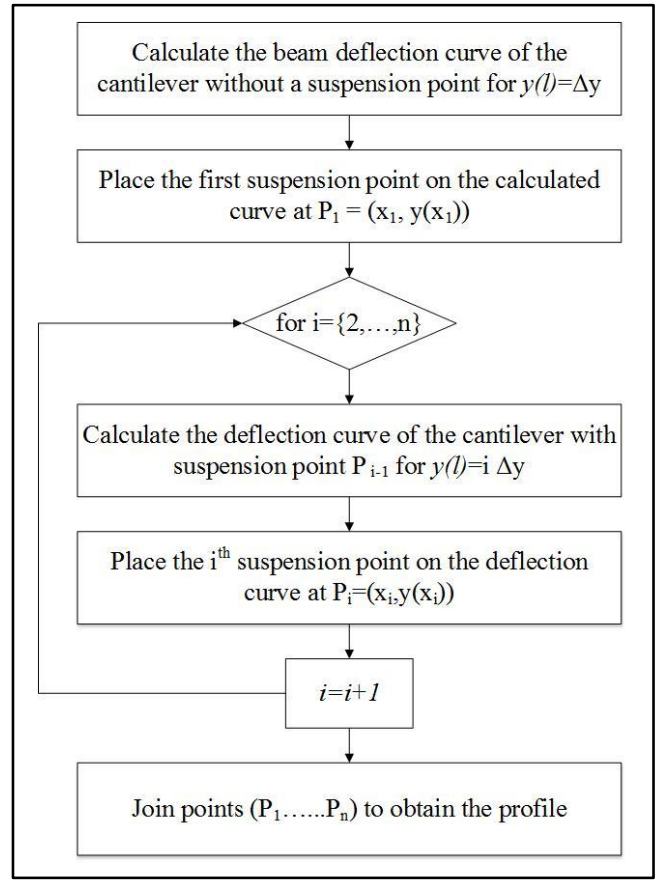

Figure 3. 14: Flow chart to obtain root profile for pre-defined nonlinearity. 
An FEA model of the designed cantilever with contact root profile was modeled using Abaqus, by modeling the cantilever as a $3 \mathrm{D}$ deformable body and the profile as a rigid body. The cantilever tip is applied with a $1 \mathrm{~mm}$ displacement in the direction to initiate contact, as illustrated in Figure 3. 15. Restoring force offered by the beam at the tip is computed from FEA simulations and it is plotted against the applied displacement to reconstruct the stiffness curve of the cantilever with rigid root profile. Since the cantilever tip is not 1-D, restoring force is obtained by taking the average of force computed on all the nodes of the cantilever tip face. The predefined load displacement curve used for generation of root profile is plotted against that obtained from FEA for comparison. Mesh density was increased until the deviation in restoring force obtained between successive mesh densities was $1.5 \%$ to ensure convergence. It was observed that the stiffness obtained from Abaqus were higher than that of pre-defined values. A stiffening behavior of the cantilever in Abaqus simulation case was observed as shown in Figure 3. 16. This can be attributed to the thin beam assumption made to compute the root profile. In fact, a deviation of $3 \%$ in linear stiffness of the beam was observed by simulating the same case without introducing the rigid contact in Abaqus model, and by setting $k_{n}=0$ in the analytical model.

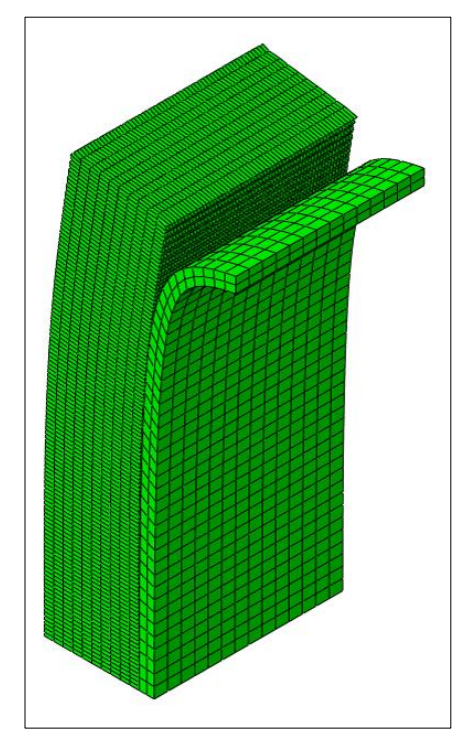

Figure 3. 15: Cantilever beam modeled in Abaqus with root profile obtained from MATLAB. 


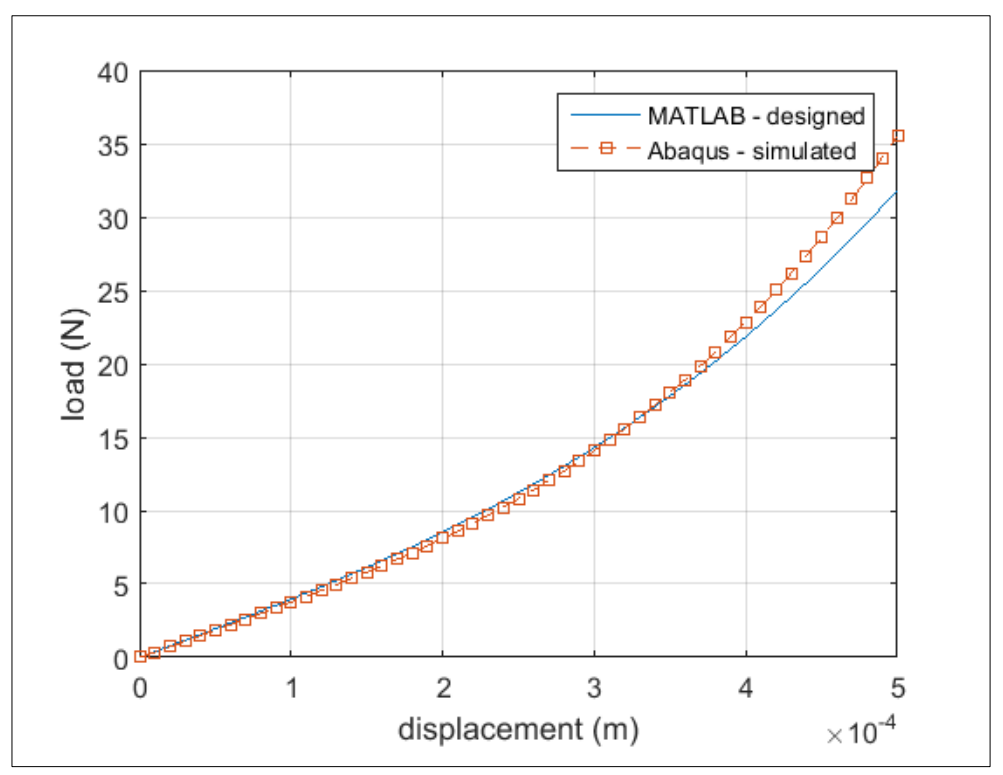

Figure 3. 16: Comparison of designed stiffness against simulation results obtained using

Abaqus.

The system demanded the addition of a heavy steel mass be attached as tip mass, to keep the mass ratio consistent with the defined parameters. The steel mass was machined with $\mathrm{CNC}$ milling, while the polycarbonate part was cut with the help of a $\mathrm{CNC}$ waterjet machine. Owing to the limitation imposed by the least diameter of cut achievable by water jet, (with abrasive) the profiles had to be fabricated separately and assembled in the unit cells after cutting the cantilevers. These were printed by using Selective Laser Sintering process and eventually assembled by press fitting to form the NLAM test-article.

Figure 3. 18 shows the first mode shape of the NLAM cantilever in the quasi-linear regime. The profile insert was required to be suppressed in SolidWorks assembly to ensure the validity of linear stiffness for computation of modal frequency. Figure 3. 17 shows the dimensions of the NLAM test-article as used for fabrication. Note that the assembly procedure illustrated in Figure 3. 19, for NLAM test-article demanded an interference fit. The assembly involved adhesion of steel mass to the cantilever and press fitting of the 3-D printed nonlinear profiles. 


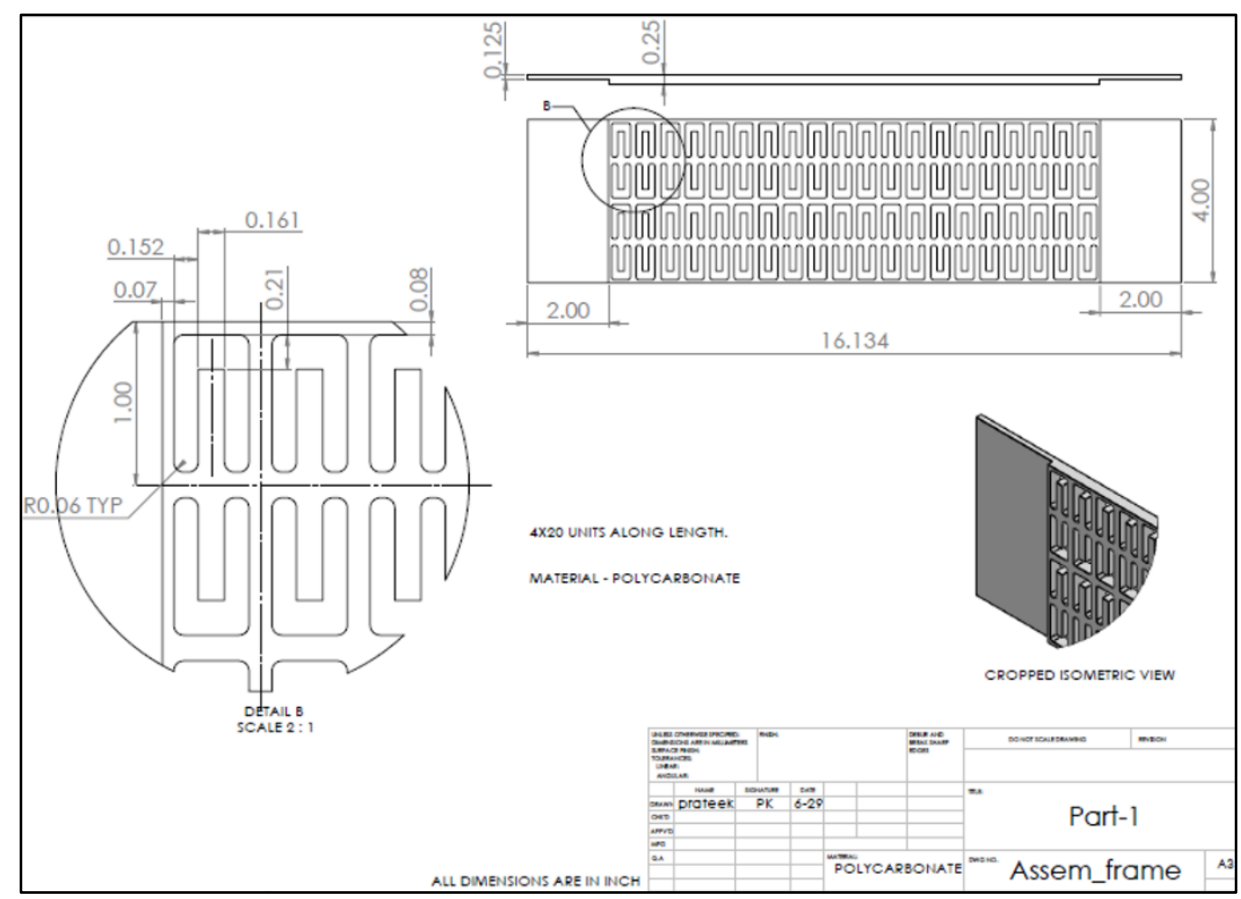

(a)

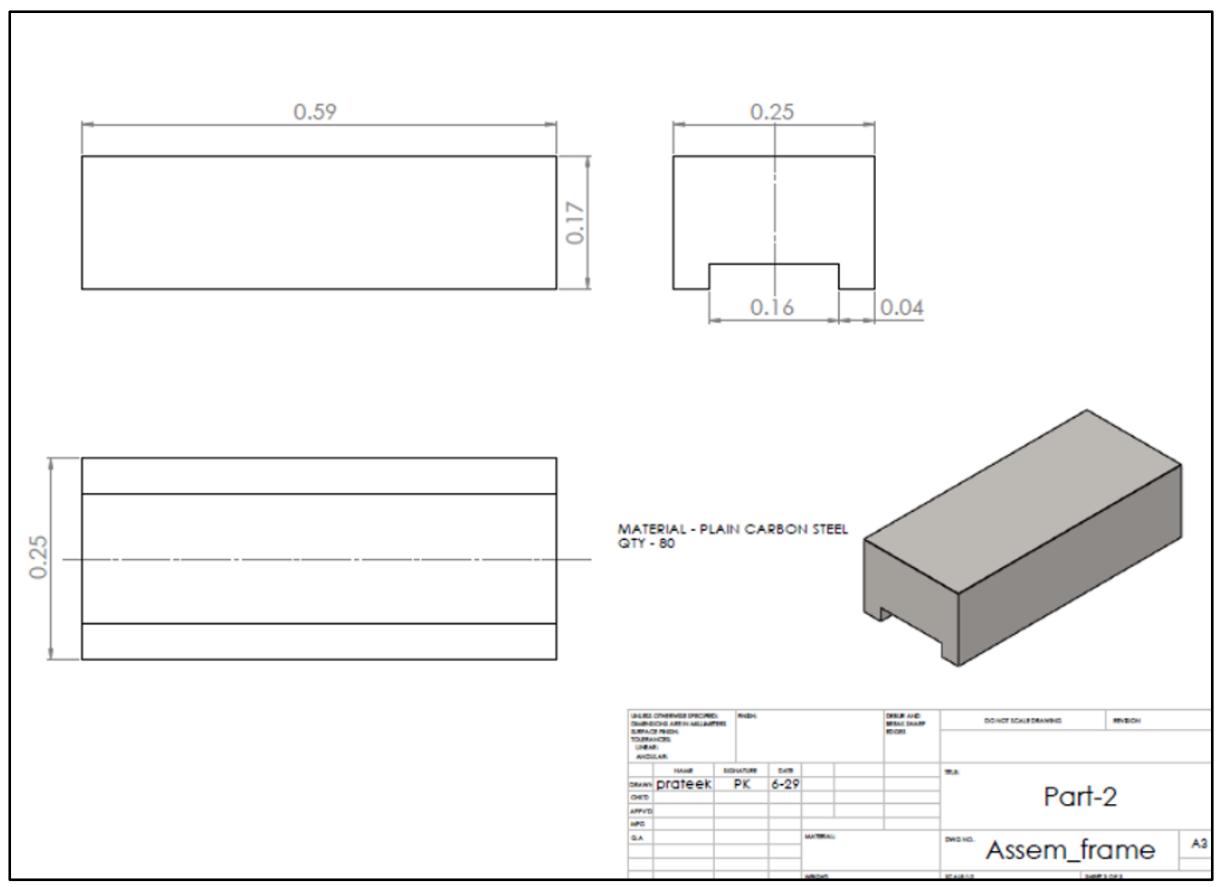

(b)

Figure 3. 17: Technical drawings to achieve linear part of the NLAM test-article (a) Polycarbonate plate with cantilever features and (b) steel tip mass. 


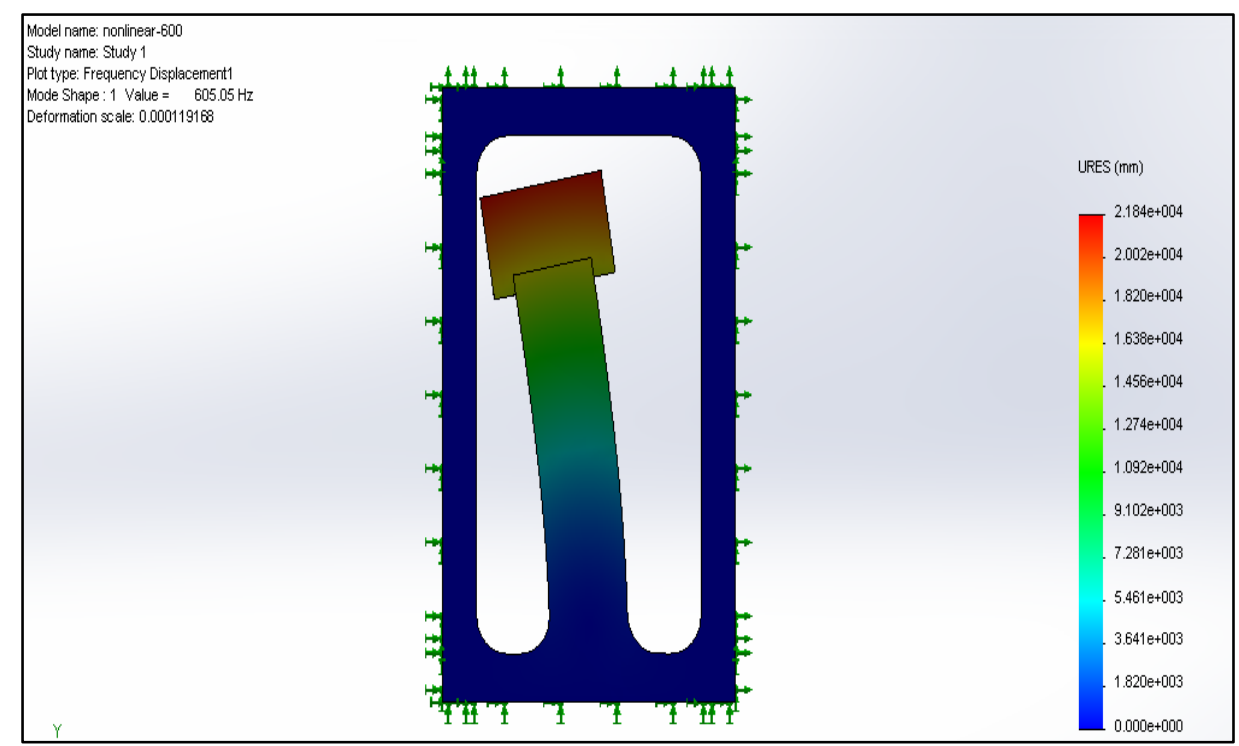

Figure 3. 18: First mode shape of designed NLAM unit cell from SolidWorks.

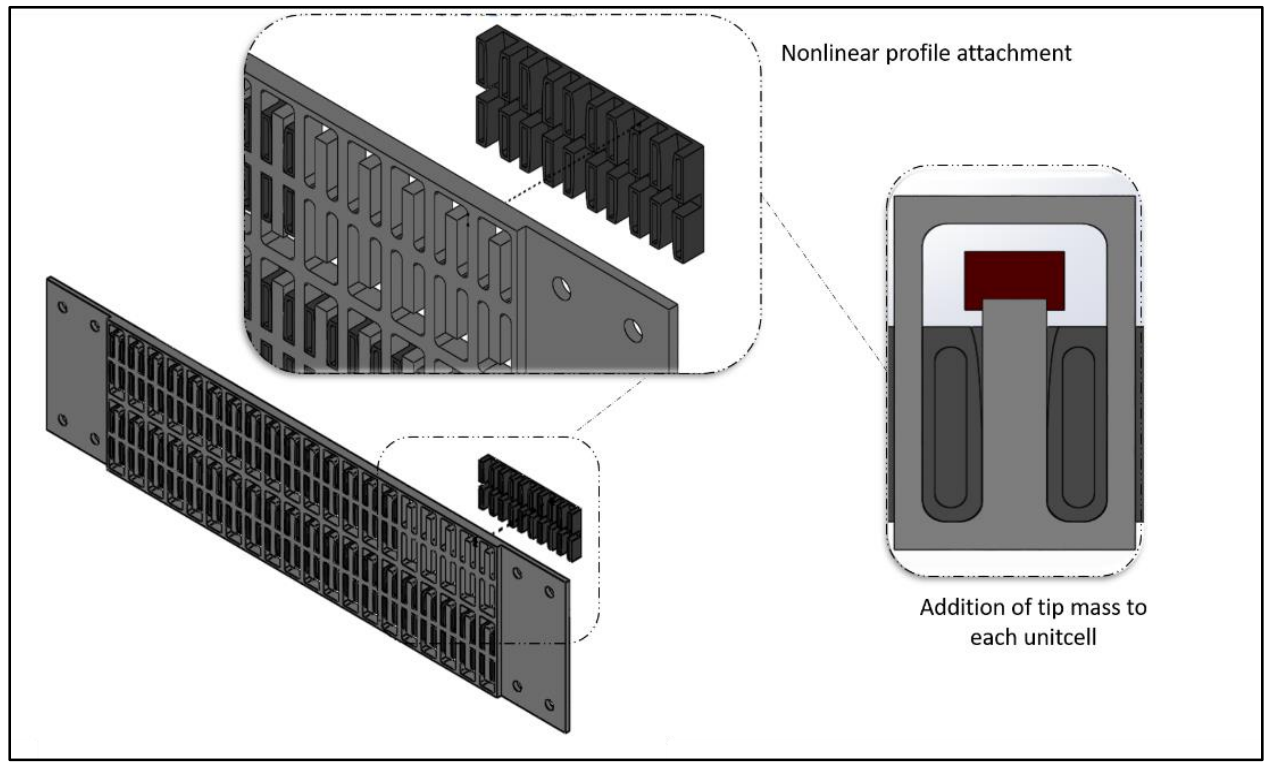

Figure 3. 19: Assembly of the NLAM test-article. 


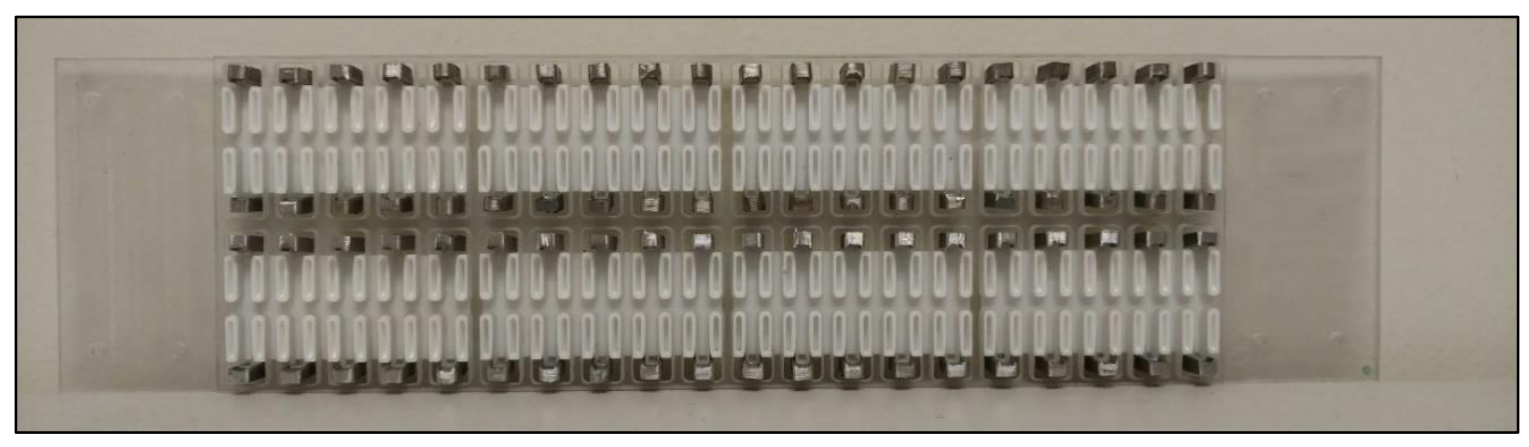

Figure 3. 20: Fully assembled NLAM test-article.

Figure 3. 20 shows the finished NLAM test-article and Figure 3. 21 represents the spread of natural frequency of the manufactured oscillators. These measurements were taken by measuring the response of each tip mass with a laser Vibrometer and computing frequency content of the time history for an impact type excitation at tip mass. The non-contact method of measurement ensures no modification of the queried system arising from instrumentation, apart from the addition of reflective tape which measures well under $0.25 \%$ of the attached mass and is assumed insignificant enough to have an appreciable effect on measured frequency due to its mass. The final assembled DBWG test-article is shown in Figure 3. 22.

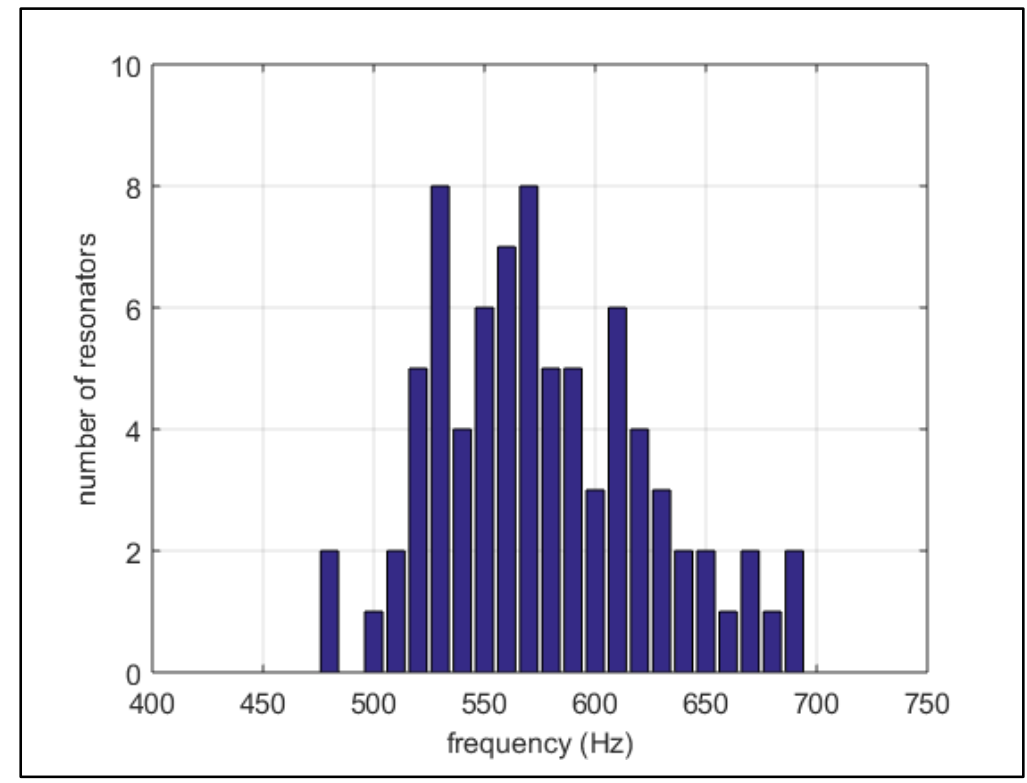

Figure 3. 21: Experimentally recorded local resonance frequencies of NLAM. 
The assembled test-article is shown in the following figure.

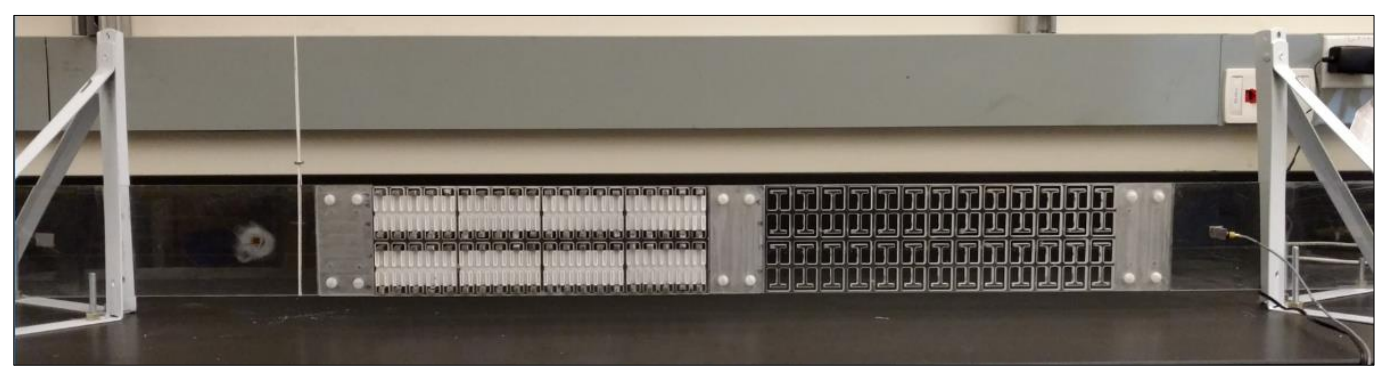

Figure 3. 22: Assembled DBWG test-article.

\subsection{Discussion of Results}

\subsubsection{Locally Resonant Acoustic Metamaterial}

The response of a point on shaker head is treated as input and a point 2 " after test-article is regarded as output. The displacement history at these points is recorded with the accelerometer mounted along the center line of the waveguide. Displacement transmissibility $\left(\mathrm{D}^{*}\right)$ is obtained by the ratio of output displacement amplitude and input displacement from the frequency spectrum.

Experimental and theoretical $\mathrm{D}^{*}$ values are plotted in Figure 3. 23 which demonstrates the bandgap and are in good correlation. A shift in the lower limit of bandgap was evident from the plots, which is due to the resonators with a natural frequency below the designed value. Simulation results indicating wave attenuation in this frequency are presented.

Time histories and frequency spectra of four cases are shown in Figure 3. 24. the four frequencies chosen were, $1000 \mathrm{~Hz}, 1100 \mathrm{~Hz}, 1250 \mathrm{~Hz}$ and $1500 \mathrm{~Hz}$, which are below, inside and above the experimentally obtained bandgap. 


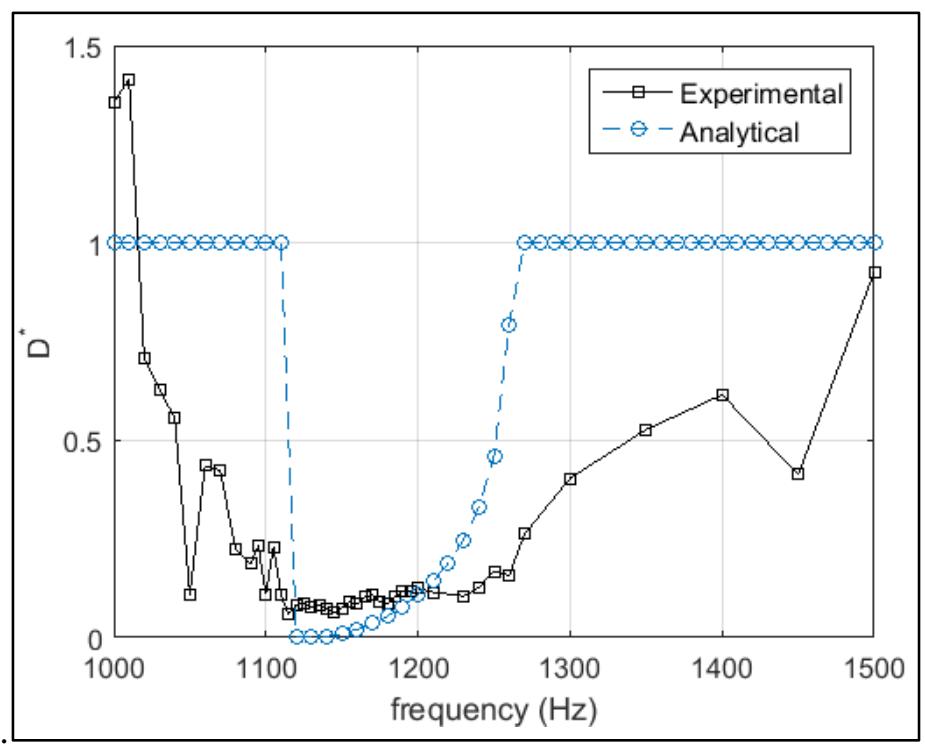

Figure 3. 23: Comparison of experimental and theoretical transmissibility curves for the LRAM test-article demonstrating the bandgap phenomenon.
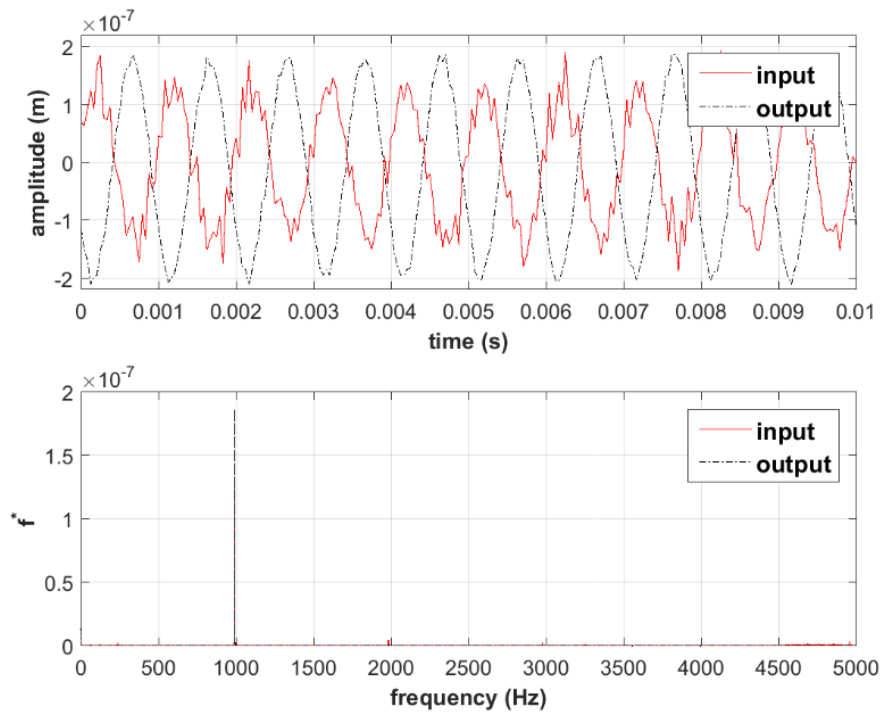

(a) 


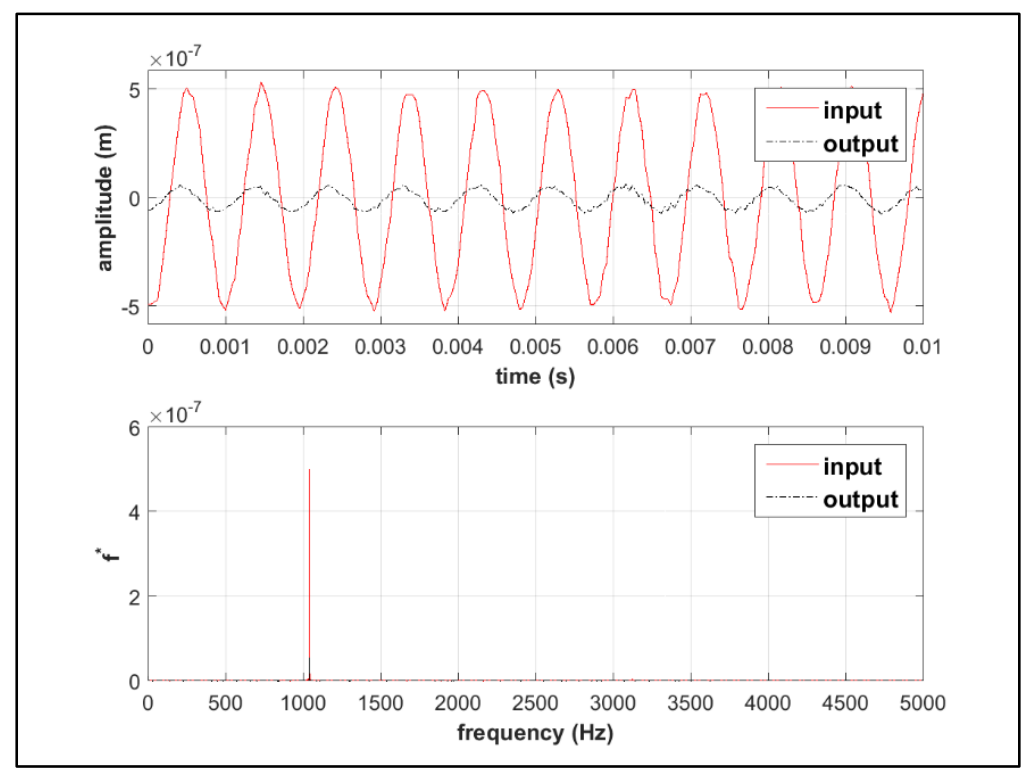

(b)

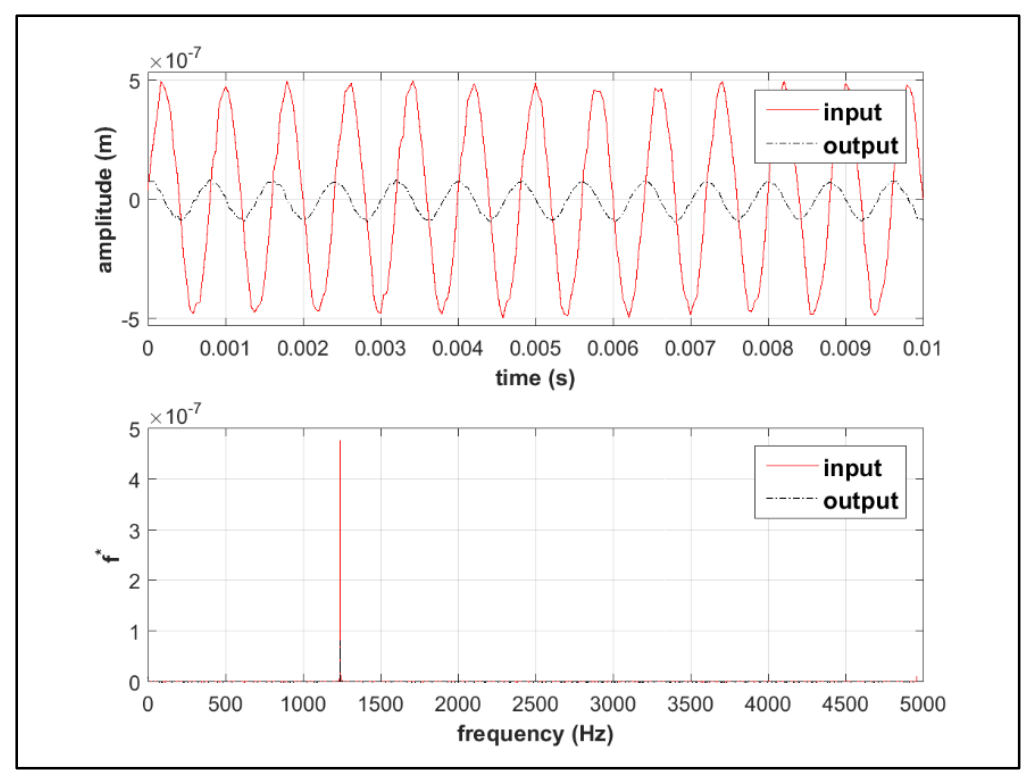

(c) 

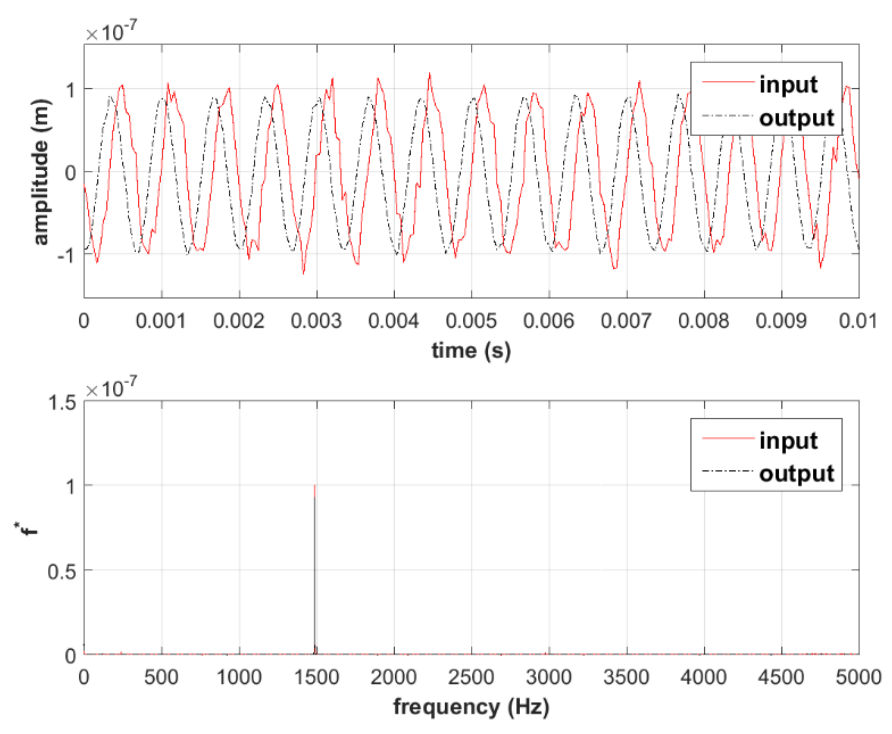

(d)

Figure 3. 24: Time history and frequency spectrum of forcing frequencies within and outside theoretical bandgap. The excitations are (a) at $1000 \mathrm{~Hz}$ which is below the lower bound, (b) at $1100 \mathrm{~Hz}$ which is within the bandgap, (c) at $1250 \mathrm{~Hz}$ also within bandgap and (d) $1500 \mathrm{~Hz}$ which is above the upper bound of the bandgap.

\subsubsection{Nonlinear Acoustic Metamaterial}

The $\mathrm{D}^{*}$ values obtained by including only the NLAM specimen in the experimental set up were plotted (Figure 3. 25). There is an evident attenuation within the theoretically predicted bandgap $(600-980 \mathrm{~Hz})$ at low amplitude, where nonlinearities are not triggered. However, a peak is encountered at $800 \mathrm{~Hz}$ which can be attributed to a spread in the manufactured local resonances. This is because theoretical predictions assume identical local resonance frequency, which is not true in the experimental scenario. The concentration of local resonance below $600 \mathrm{~Hz}$ explains the early initiation of experimental bandgap compared to theoretical predictions. 


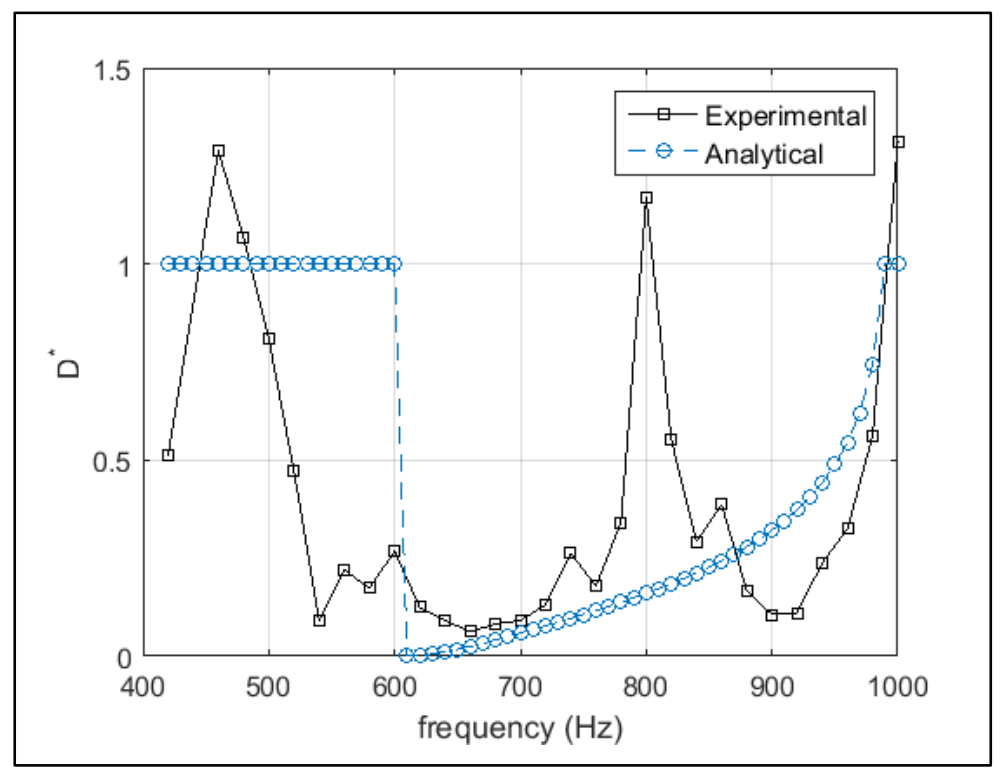

Figure 3. 25: Comparison of experimental and analytical transmissibility curves for the NLAM test-article demonstrating bandgap phenomenon in the linear regime.

\subsubsection{Direction-Biased Waveguide Device}

The DBWG test-article was obtained by joining the NLAM and LRAM test-articles in series. Similar procedure was followed to record the displacement responses and to obtain $\mathrm{D}^{*}$. The testarticle was subjected to amplitude and direction-bias testing. It requires the waves to be transmitted in two opposite directions along the test-article. The shaker, being a heavy component was kept fixed. Hence, the test-article was reversed to reverse the direction of the wave. The configuration where NLAM is to the left is chosen as a reference. Therefore, the configuration with NLAM to the left is considered to be forward and LRAM to the left is considered reverse. In both the directions, low and high amplitude excitations were applied. Thus, four cases were obtained for each frequency and transmissibility curves are shown in Figure 3. 26. The D* values were obtained from a set of three experiments and displayed a maximum deviation of $8 \%$ between successive attempts. 


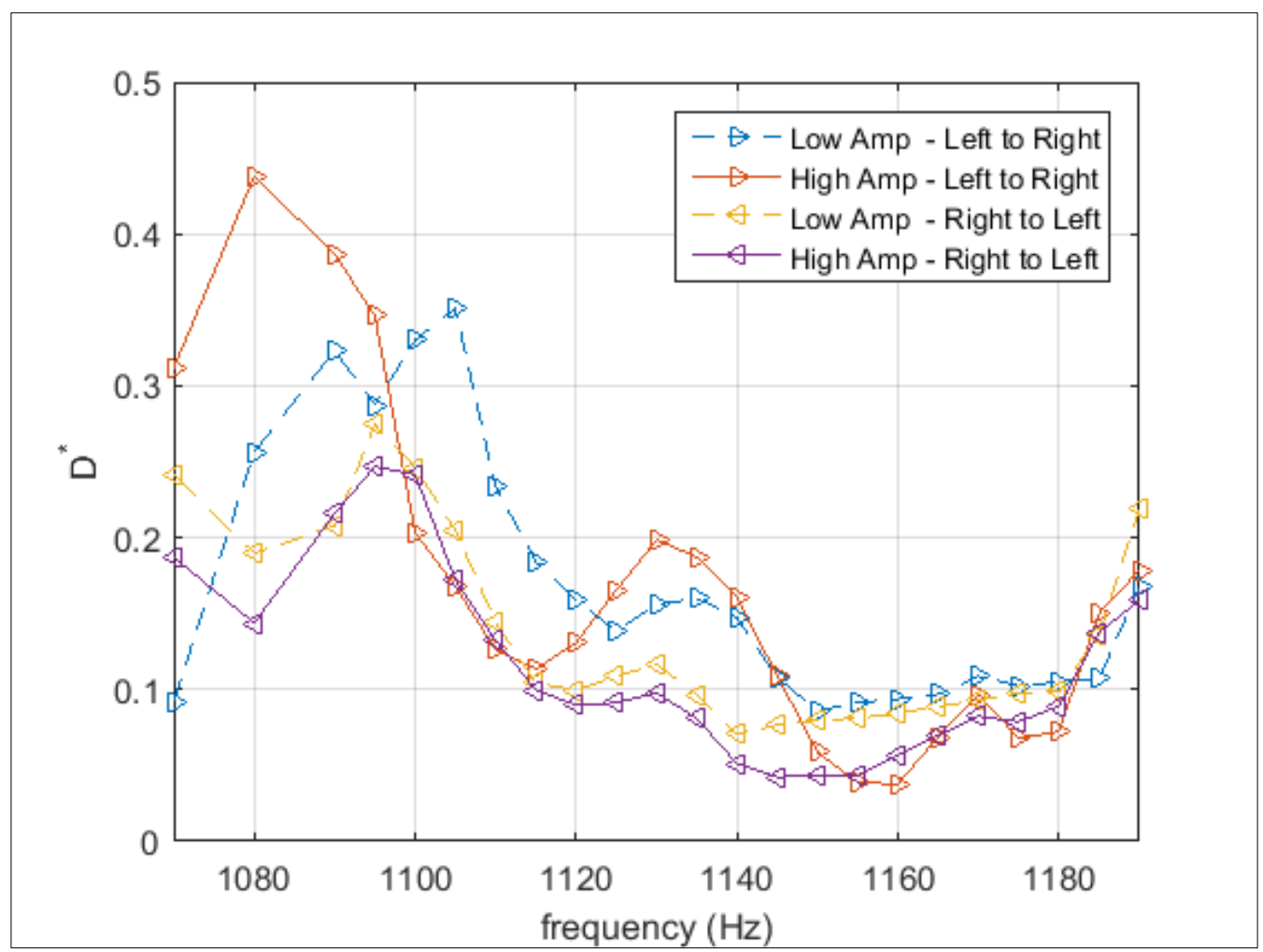

Figure 3. 26: Transmissibility curves for the final test-article (NLAM and LRAM joined) for the four cases covering both directions and amplitudes of excitation.

The design of specimen was based on the results obtained from simulations that display an amplitude-dependent direction bias in wave propagation. Therefore, a harmonic wave at $\Omega=1.9$ should experience attenuation along either direction at low amplitude. However, the same wave at higher amplitude propagates while traversing along left to right direction. At low amplitude, the wave attenuates.

The manufactured specimen displayed considerable spread in frequency as compared to the design. Therefore, instead of querying at a single frequency, a range of frequencies in the neighborhood of predicted value was considered.

The D* vs frequency plots obtained from the experiments is shown in Figure 3. 26. In the vicinity of $1080 \mathrm{~Hz}$, an interesting trend can be observed. This region displays direction-bias as predicted by simulations, although at a lower frequency. This supports the notion of a larger relative 
propagation compared to the other cases at the same frequency. A closer examination of the time signals and frequency spectra for these cases show $100 \%$ increase in propagation as may be expected depending on the numerical study. The amplitude and frequency plots for a comparative study are shown in Figure 3. 27.

A strong attenuation arising from the LRAM bandgap is prominent in the 'right to left' case. In the neighborhood of the designed frequency of $1140 \mathrm{~Hz}(\Omega=1.9)$, there is a minor increase in $\mathrm{D}^{*}$ in accordance with the direction-bias mechanism. This deviation from expected result could be a result of - i) frequency spread; ii) manufacturing defects; iii) different hardening mechanism from designed.

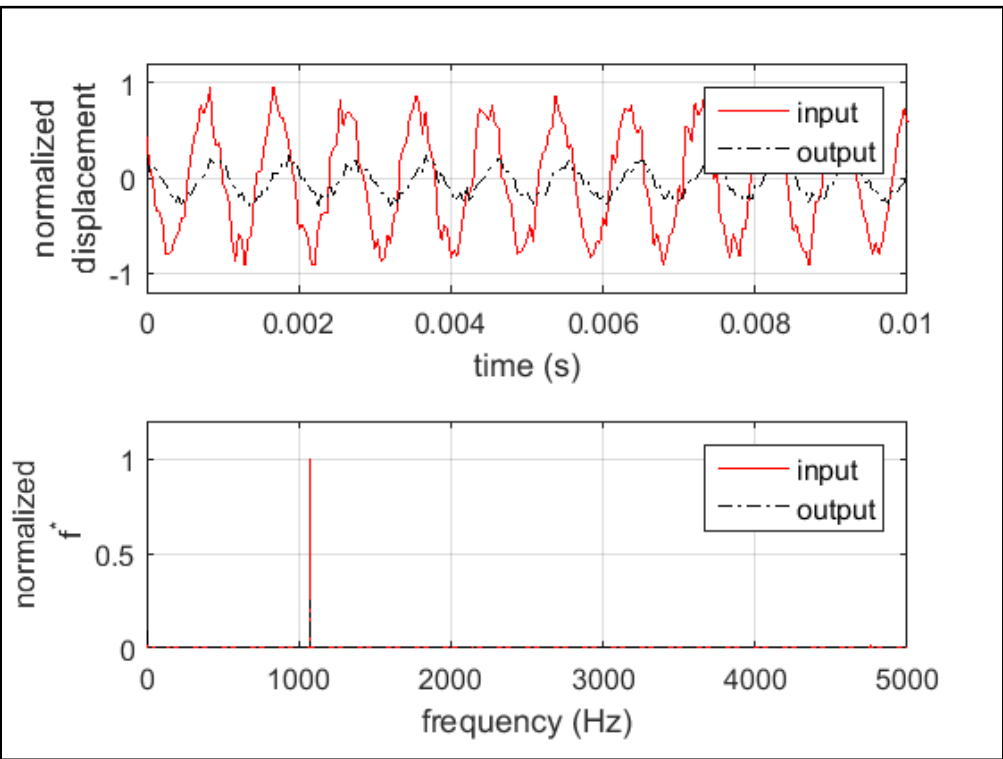

(a) 


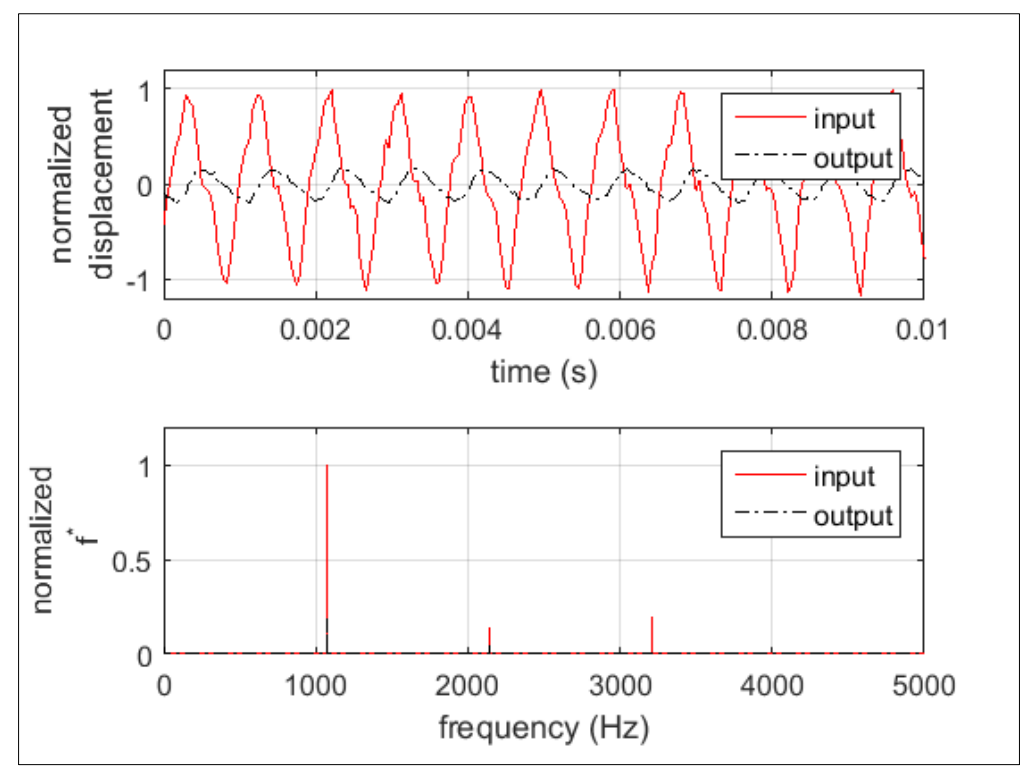

(b)

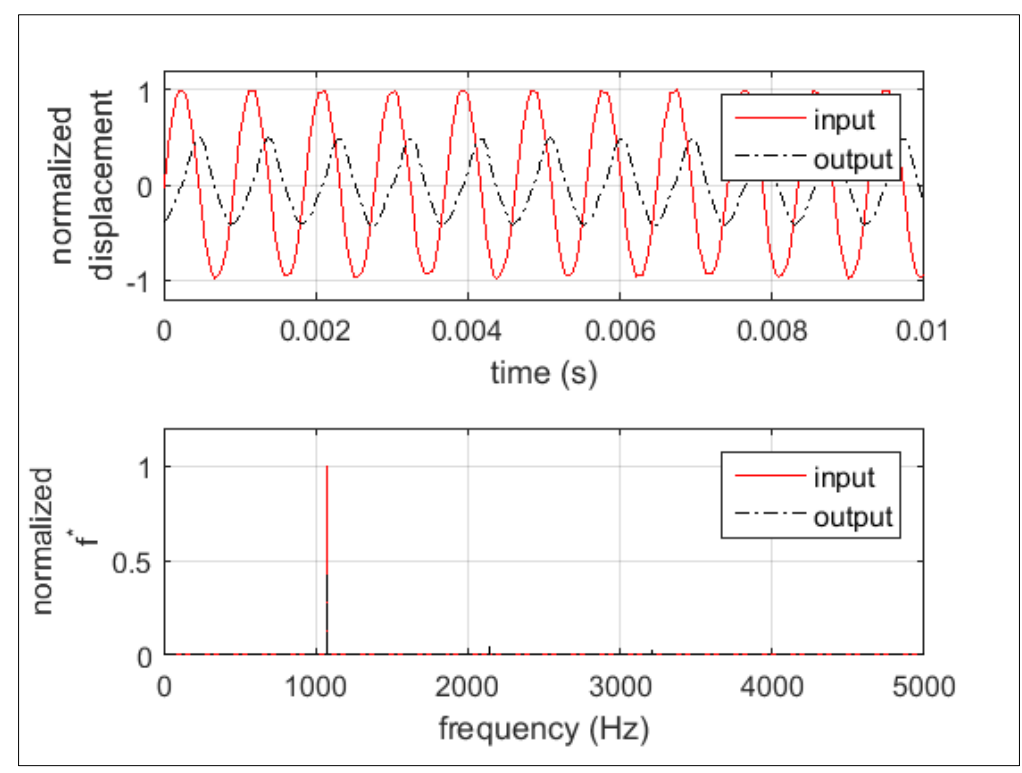

(c) 


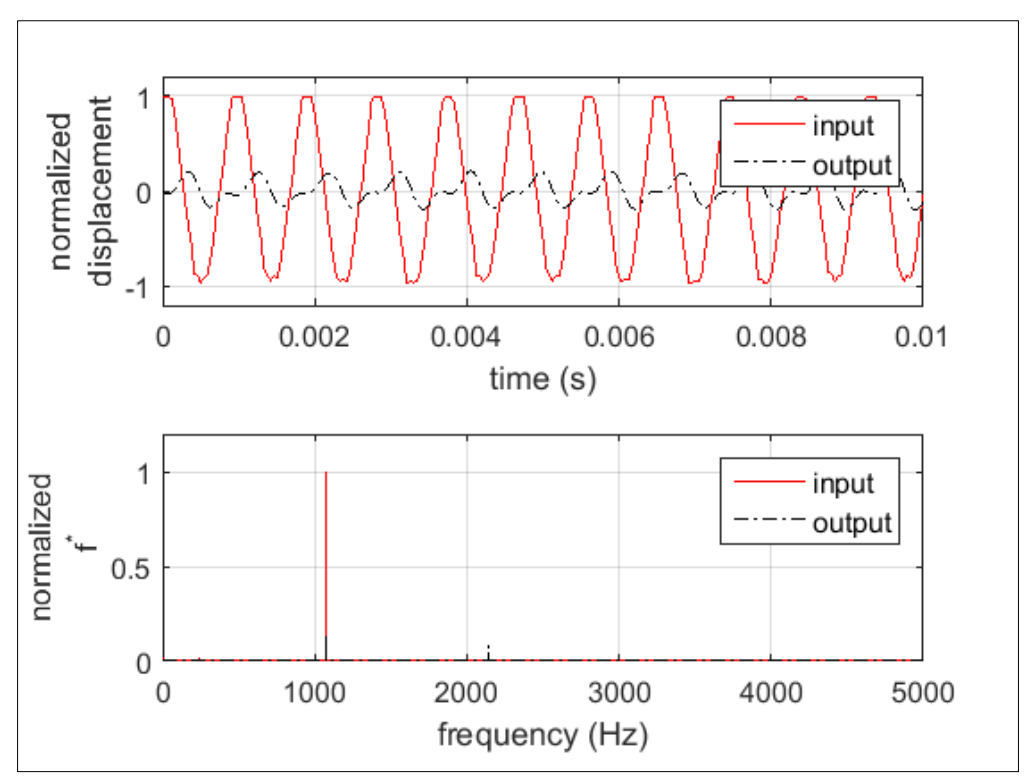

(d)

Figure 3. 27: Response of the test-article at $1080 \mathrm{~Hz}$, at different conditions - Low amplitude (a) Left to Right, (b) Right to Left and High amplitude (c) Left to Right, (d) Right to Left direction of traverse.

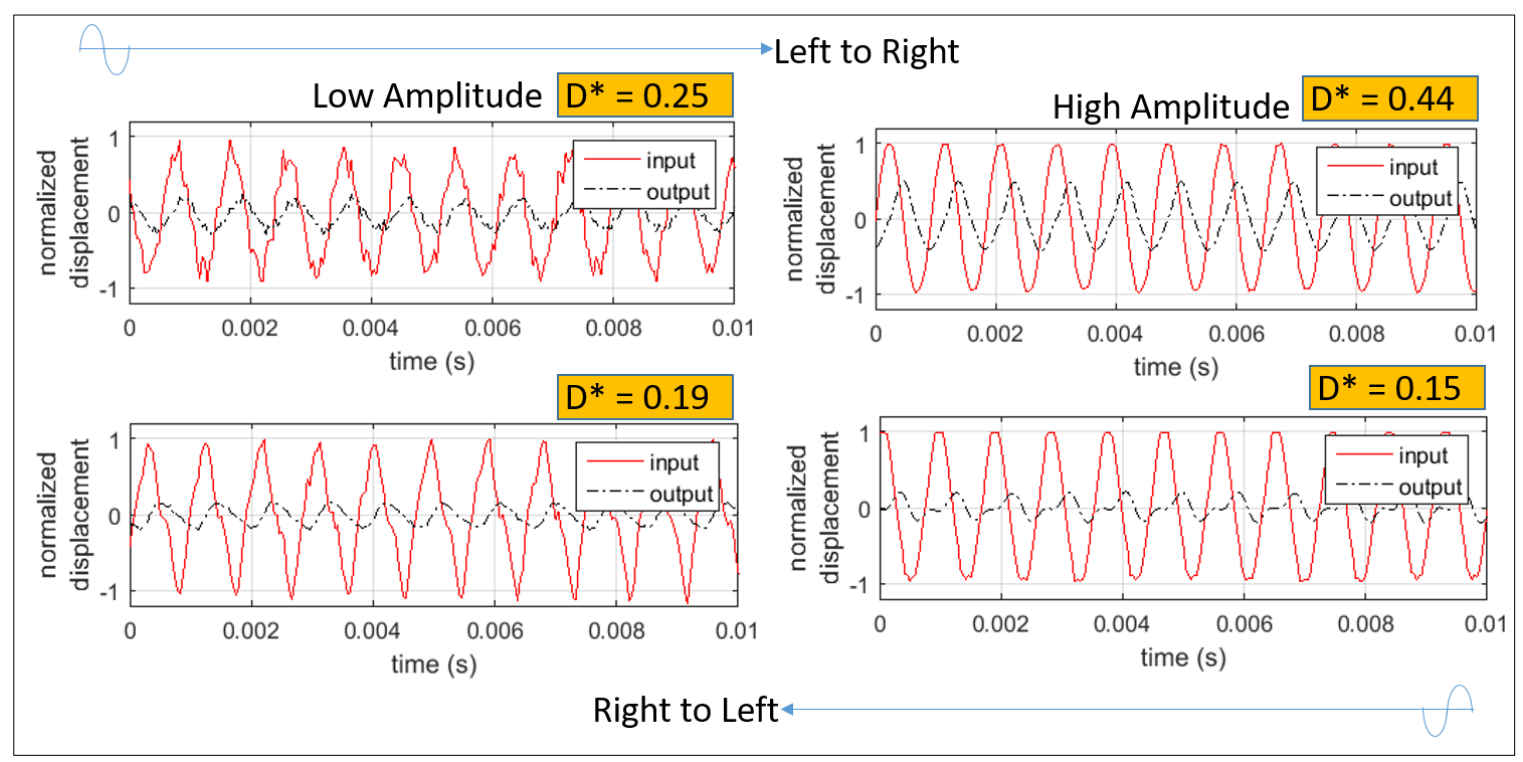

Figure 3. 28: Direction bias effect observed at $1080 \mathrm{~Hz}$. 


\subsection{Summary}

The experimental set up was sized depending on the planned set of experiments. Detailed testarticle was designed and Abaqus simulations were conducted to verify the designed nonlinear stiffness of the NLAM part. Characterization of the waveguide, LRAM and NLAM were carried out and the individual bandgaps of the test-articles were measured. The bandgaps were observed in good agreement with theoretical predictions. However, deviations arising from fabrication process were observed. The proposed 'direction-biased waveguide' test-article was assembled and tested. Significant trend supportive of proposed direction-bias effect was observed at a frequency lower than designed value. A minor direction bias was observed in close vicinity of expected frequency. 


\section{CHAPTER IV}

\section{INERTANT ACOUSTIC METAMATERIALS}

\subsection{Introduction}

Acoustic metamaterials exhibit unusual dynamic properties not readily realizable in natural or other man-made structural materials. Modernization of the society and human demands have placed a great demand on obtaining materials with improved and diverse performance characteristics. In such a scenario, it is inevitable to explore diverse combinations of established materials or even include new elements. The inerter is one such example of a new element capable of displaying a modified dynamic mass. The effect of adding the inerter in various configurations of discrete element lattice is investigated in the subsequent sections, to explore the behavior of the resulting 'Inertant Metamaterials'.

Lumped parameter model with locally resonant attachments [12] is discussed first, as this forms the basis of all subsequent analysis. The method is applied to a few configurations of inertant metamaterials with the inerter included locally and in host material (lattice). Both series and parallel combinations of the inerter and stiffness element are considered to expand the domain of study. Their characteristics such as bandgap width, the degree of attenuation, nature of propagation modes and limiting behavior are investigated and compared against locally resonant AM. Results obtained in the form of dispersion relation, are used to postulate wave manipulation devices. The potential to realize novel dynamic behavior is demonstrated by postulating a complete longitudinal wave inhibitor using linear combinations of inertant AM configurations. 


\subsection{Analytical Models}

An arbitrarily chosen unit cell along an infinite 1-D mass-spring lattice is considered and assumed to be the $j^{\text {th }}$ unit cell. By assuming a harmonic forcing of frequency $\omega$, the equations of motion for this unit cell are set up. The system is considered to be linear and hence the displacement response for the mass is assumed to be harmonic. The analysis model is illustrated by applying to an LRAM chain as detailed in [12]. The inertant metamaterial cases that follow are represented similarly as a monoatomic mass-spring model with each unit cell consisting of an 'effective mass'. The effective mass is conceived in order to capture the effect of attaching a second internal degree of freedom. This method is applied to mass-spring chains having inerter introduced first in local attachment and then in the lattice. The inerter is combined with the stiffness in parallel and series attachments. By including an inerter with lattice spring requires the modeling procedure to be slightly modified and an effective stiffness is introduced. The following models are considered for analysis in the following

a. Inertance in local attachment
i. Only inertance
ii. Inertance in parallel
iii. Inertance in series

b. Inertance in lattice
i. Inertance in parallel
ii. Inertance in series

LRAM is one of the limiting cases for all the models mentioned above, except the 'only inertance' case. 
4.2.1 Locally Resonant Acoustic Metamaterial and its Effective-Mass Model

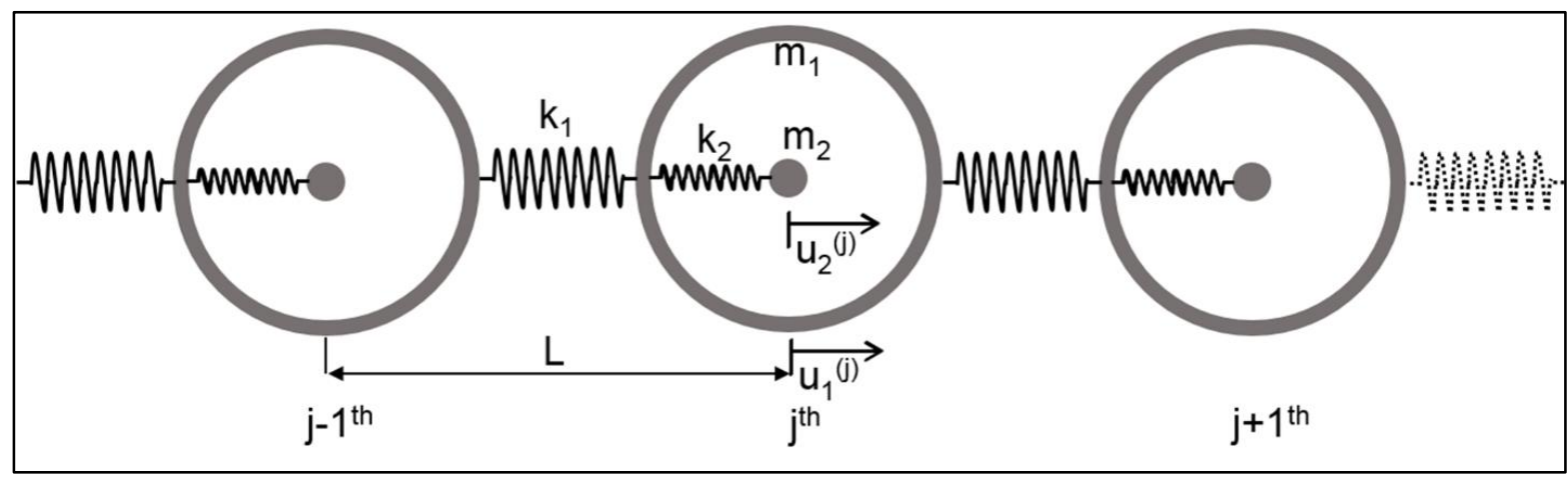

(a)

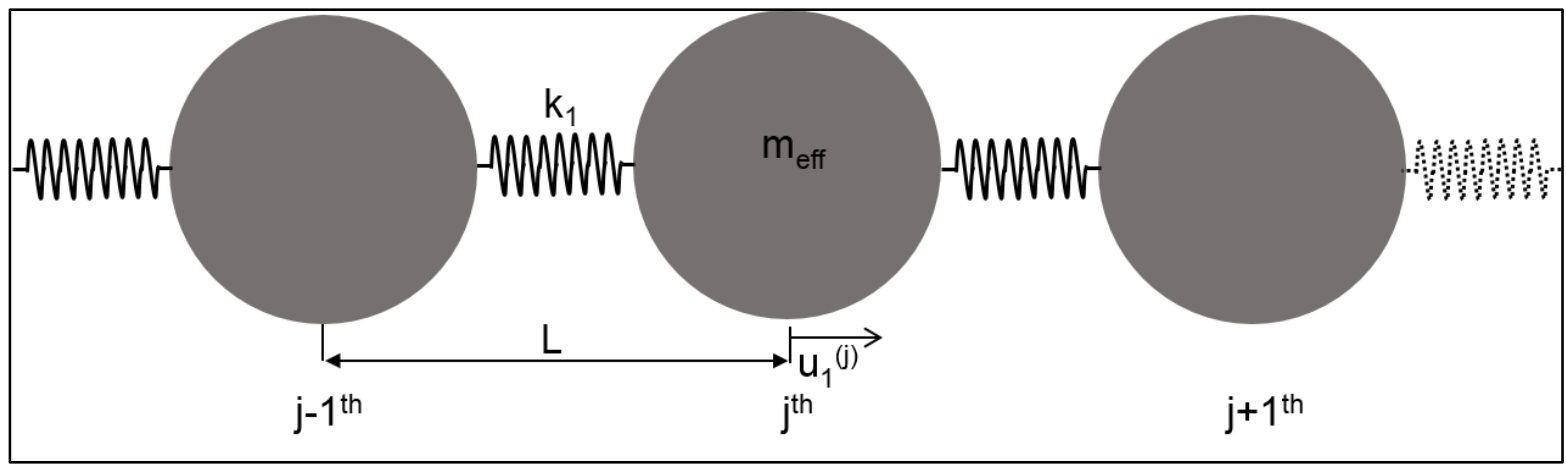

(b)

Figure 4. 1: (a) Discrete mass-spring lattice model for a locally resonant acoustic metamaterial and (b) its effective-mass model.

This section provides a brief introduction to the general method used for subsequent analytical explorations of the inertant metamaterials. Consider an infinite mass-in-mass chain as shown in Figure 4. 1 (a) which represents an LRAM that consists of locally resonant inclusions within a host material. A 1-D lattice chain is considered and displacements only along the same direction are regarded. A single unit cell consists of external masses $\left(m_{1}\right)$ connected by springs of stiffness $\left(k_{1}\right)$ representing the host material and internal masses $\left(m_{2}\right)$ connected by an internal spring of 
stiffness $\left(k_{2}\right)$ to the external mass to form a resonating local attachment. This 1-D lattice is represented as a monoatomic chain with each mass designated by an effective mass. This effective mass is required to capture the effect of both the degrees of freedom. The equations of motion for the monoatomic chain and the original lattice are set up for an assumed harmonic forcing. From the argument that to be representative of each other, both these systems should have the same displacement response for the known applied forcing. This condition is applied to obtain the expression of effective mass given as

$$
m_{e f f}^{L R A M}=\left(m_{s t}+\frac{m_{2} \Omega^{2}}{\left(1-\Omega^{2}\right)}\right)
$$

where $m_{s t}=m_{1}+m_{2}$ is the static mass and $\Omega=\omega / \omega_{0}$ is the normalized excitation frequency with $\omega_{0}=\sqrt{k_{2} / m_{2}}$ being the local resonance frequency. A parametric setting of $\left[m_{1}, m_{2}, k_{1}, k_{2}, L\right]=[10,9,100,10,1]$ is used for generating the plots in Figure 4. 2. Querying for a negative effective mass (Figure 4. 2 (a)) in Equation 4.1 results in the condition given by

$$
1<\Omega<\sqrt{1+\theta_{21}}
$$

where $\theta_{21}=m_{2} / m_{1}$ is the mass ratio. Setting up the equations of motion for the $j^{\text {th }}$ unit-cell, the dispersion relation for the LRAM can be obtained as

$$
q L=\alpha+i \beta=\cos ^{-1}\left[1-\frac{m_{e f f}^{L R A M} \omega^{2}}{2 k_{1}}\right]=\cos ^{-1}\left[1-\frac{m_{e f f}^{L R A M} \Omega^{2}}{2 m_{1}}\right]
$$

where $q L$ is the normalized wavenumber and $\alpha$ and $\beta$ are its real and imaginary parts respectively. The real and imaginary parts of wavenumber are plotted against the normalized excitation frequency in Figure 4. 2 (b) and (c). As can be seen from these plots and the expression for the wavenumber in Equation 4.3, the frequency range of effective-mass negativity corresponds to the wavenumber becoming purely imaginary $(q L=i \beta)$ leading to attenuation of harmonic waves of frequencies within this range, where $\beta$ is termed the attenuation factor. Thus the frequency range of effective-mass negativity corresponds to a bandgap for harmonic wave propagation with the LRAM. There are two propagating modes - acoustic and optical modes 
respectively below and above the bandgap while an additional bandgap region appears above the optical mode due to the discretization employed to obtain the lattice model for the LRAM. The cut-off frequency at which the discretization bandgap begins is given by Equation 4.4. This is obtained by querying for values of $\omega$ that result in complex value for $q L$ from Equation 4.3.

$$
\omega_{c o}^{L R A M}=2 \sqrt{\frac{k_{1}}{m_{e f f}^{L R A M}}}
$$

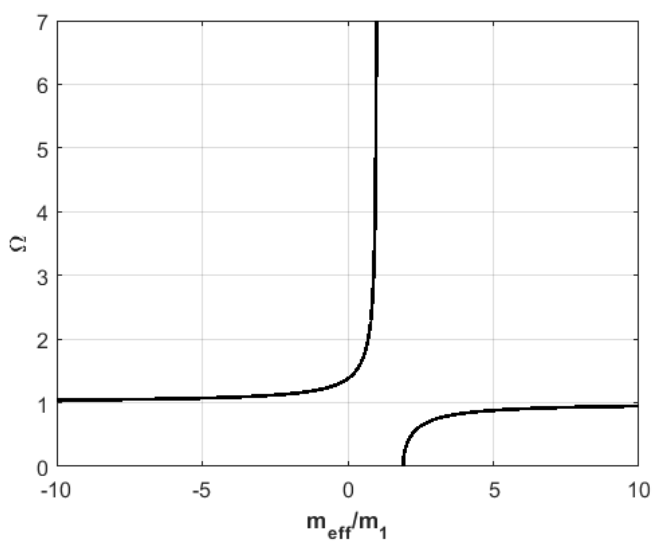

(a)

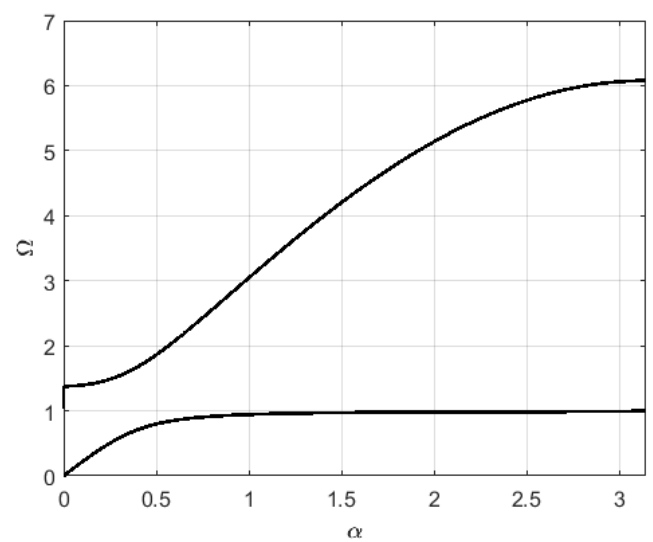

(b)

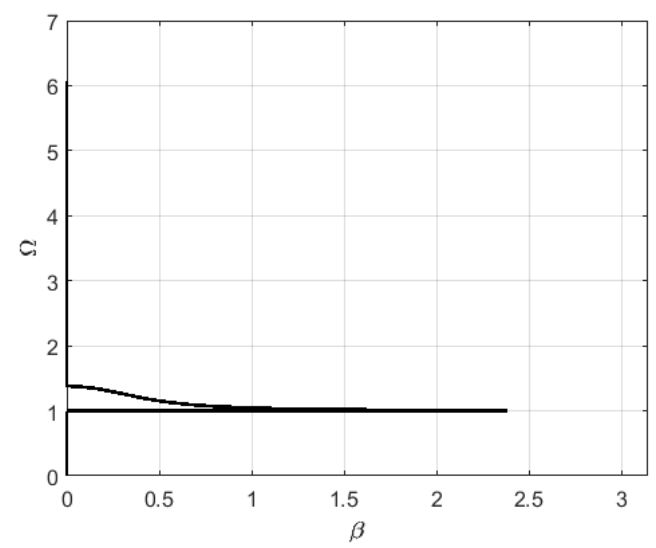

(c)

Figure 4. 2: (a) Normalized effective-mass, and (b) real and (c) imaginary parts of the wavenumber versus normalized excitation frequency for the LRAM. 
Thus the effective-mass model provides a simple means to characterize the longitudinal elastic wave propagation behavior of AM configurations having different local attachments or lattice elements. This approach is used for various inertant AM configurations to obtain dispersion behavior. In each case, the effect of inertance on propagation modes and bandgaps along with any special interactive conditions and device implications are examined.

\subsubsection{Inertant Acoustic Metamaterial Configurations}

The dispersion behavior of acoustic metamaterial configurations having inerters in either local attachments or in the lattice is examined using the effective-mass model for their discrete element representations. For each case, the effective-mass, real and imaginary parts of the wavenumber and the variation of propagation characteristics with the value of inertance is obtained and discussed. The LRAM is considered as a reference configuration to compare relative performance. For all configurations considered, a parametric setting of $\left[m_{1}, m_{2}, k_{1}, k_{2}, L\right]=$ $[10,9,100,10,1]$ is used.

Three different configurations with inerters in the local attachment are considered. These are the wholly inertant (WI), parallel inertant (PI), and series inertant (SI) attachment cases.

\subsubsection{Wholly Inertant Attachment}

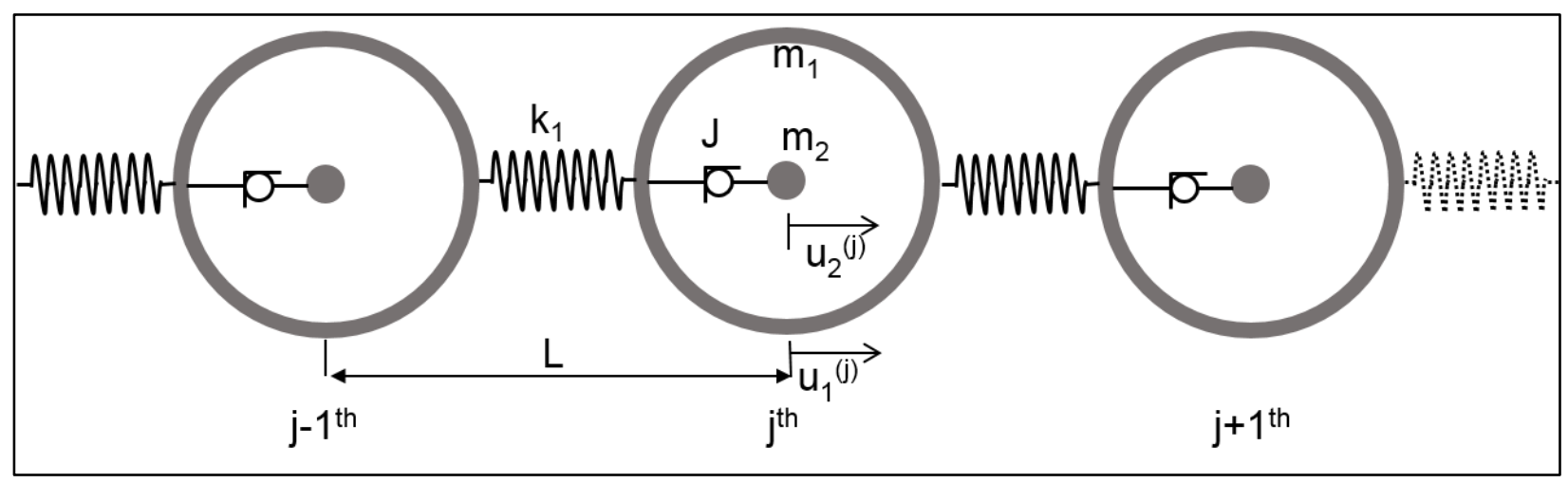

Figure 4. 3: Acoustic metamaterial with purely inertant local attachments.

The wholly inertant attachment configuration is shown in Figure 4. 3. Throughout this study, a circle within a quadrant as shown in the figure is used as the symbol for the inerter. In 
comparison to the locally resonant case, the internal spring is replaced by an inerter of inertance, $J$. Setting up equations of motion for this model, its effective-mass can be derived as

$$
m_{e f f}^{W I}=\left(m_{1}+\frac{\mathrm{J} m_{2}}{m_{2}+\mathrm{J}}\right)
$$

It must be noted that the presence of an inerter in a harmonically excited system is identical to that of a spring with a negative stiffness proportional to the square of the excitation frequency since the force across an inerter

$$
f_{J}=J\left(a_{2}-a_{1}\right)=-\omega^{2} J\left(u_{2}-u_{1}\right)
$$

where $a_{i}$ and $u_{i}$ are the acceleration and displacement at terminal $i$ of the inerter. The dispersion relation for the effective-mass model for the WI case is given by the following equation

$$
q L=\alpha+i \beta=\cos ^{-1}\left[1-\frac{m_{e f f}^{W I} \omega^{2}}{2 k_{1}}\right]=\cos ^{-1}\left[1-\frac{m_{e f f}^{W I} \Omega^{2}}{2 m_{1}}\right]
$$

where $q L$ is the normalized wavenumber, and $\Omega=\omega / \omega_{1}$ for this case is the normalized frequency with $\omega_{1}=\sqrt{k_{1} / m_{1}}$ which is the lattice frequency.

It is noted that the effective-mass is frequency-independent and is only a function of the inertance $J$ and the lattice and attachment masses. Depending on the value of $J$, the inerter augments participation of the local attachment mass with the lattice mass. Figure 4.4 (a) shows the variation of $m_{e f f} / m_{1}$ with $J / m_{1}$. As the value of $J$ increases, $m_{e f f}$ tends to $m_{1}+m_{2}$ indicating the complete participation of attachment mass $m_{2}$ with the lattice for a very large value of $J$.
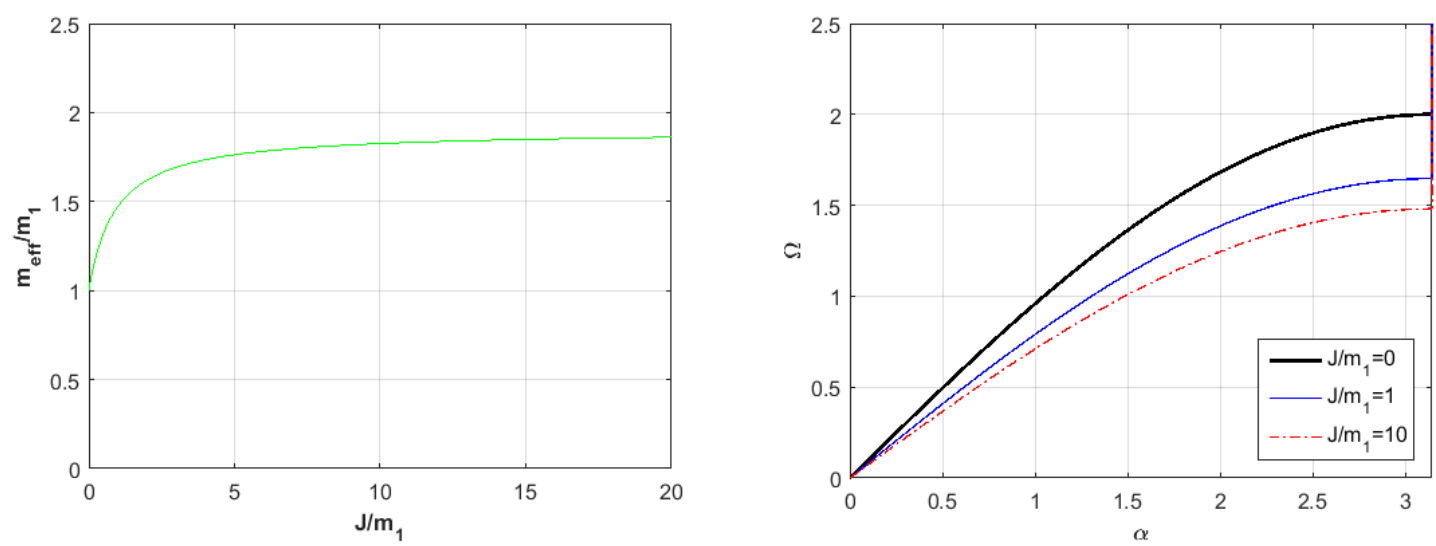


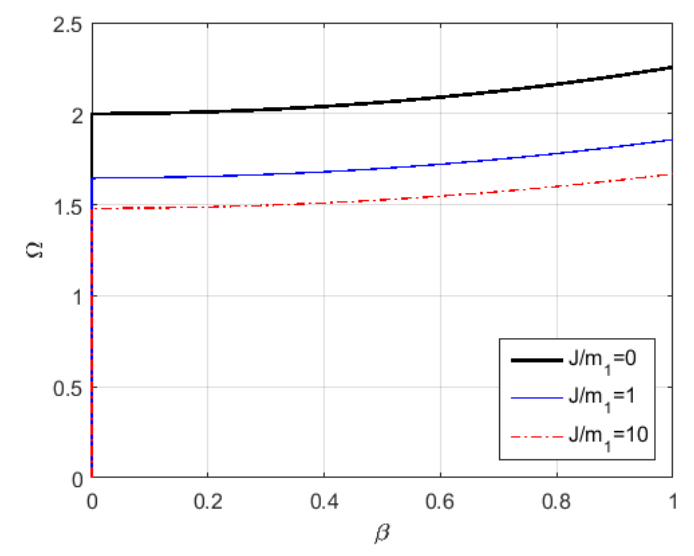

(c)

Figure 4. 4: (a) Normalized effective-mass versus normalized inertance and (b) real and (c) imaginary parts of wavenumber versus normalized excitation frequency.

The presence of a wholly inertant local attachment shifts the dispersion curve of the lattice model to lower frequencies depending on the value of inertance, $J$ in the attachment. The shifts in the real and imaginary parts of the wavenumber with increasing value of $J$ are shown in Figure 4. 4 (b) and (c) respectively. For a very small value of $J$, the system behaves as a monatomic lattice with a lattice mass of $m_{1}$ while for a very large value of $J$, the system switches to a monatomic lattice with a lattice mass of $m_{s t}$. Setting the condition for a purely imaginary wavenumber, the cut-off frequency arising from the lattice discretization can be obtained as

$$
\omega_{c o}^{P I}=2 \sqrt{\frac{k_{1}}{m_{e f f}^{P I}}}
$$

Thus, by varying the value of $J$, the acoustic metamaterial with wholly inertant local attachments can behave as a low-pass filter with a varying cut-off frequency given by Equation 4.8 due to the tunable participation of the attachment mass in the lattice dynamics. 


\subsubsection{Parallel Inertant Attachment}

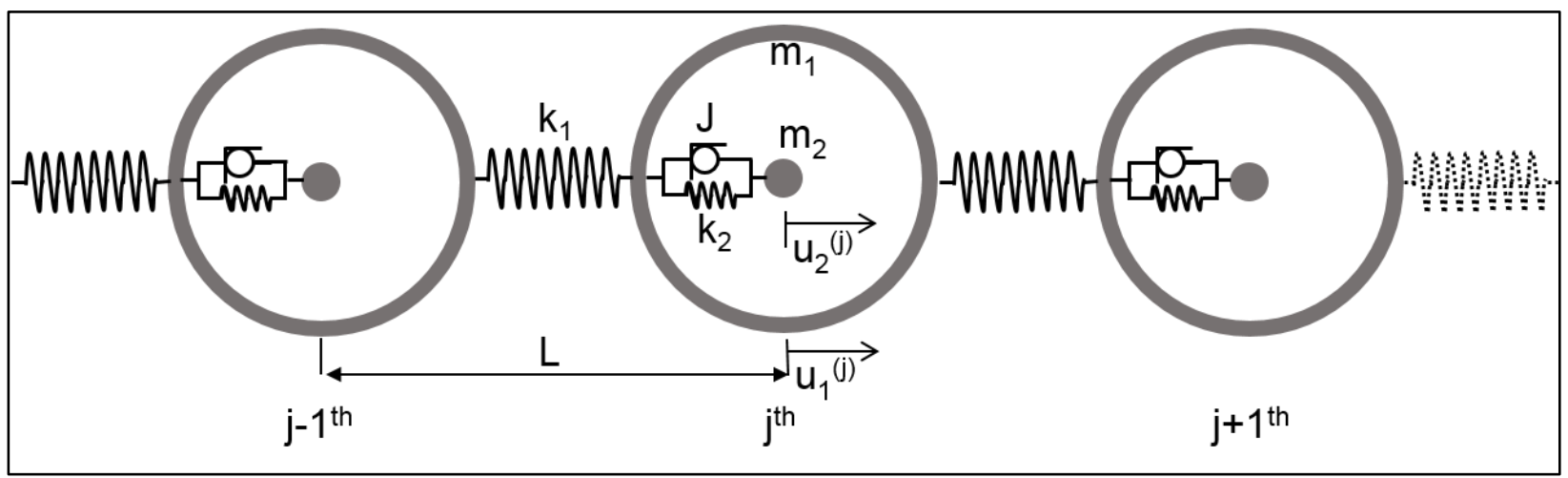

Figure 4. 5: Acoustic metamaterial with local attachments having inerter in parallel with the spring.

An acoustic metamaterial lattice model with inerters in parallel to the spring in the local attachment is shown in Figure 4. 5. This configuration is referred to as the parallel inertant attachment case. The effective-mass for this case is obtained as

$$
m_{e f f}^{P I}=\left[m_{s t}-\frac{m_{2}^{2}}{m_{2}+\left(J-\frac{m_{2}}{\Omega^{2}}\right)}\right]
$$

where $\Omega=\omega / \omega_{0}$ for this case is the normalized excitation frequency with $\omega_{0}=\sqrt{k_{2} / m_{2}}$ which is the local resonance frequency. As can be noted in Figure 4. 6 (a), the presence of the inerter in parallel to the internal spring both shifts to lower frequencies as well as diminishes the frequency range of effective-mass negativity with increase in the value of $J$. For a very small value of $J$, the system behaves essentially as an LRAM with an attachment mass of $m_{2}$, while for a very large value of $J$, the behavior tends to that of a monatomic lattice with a lattice mass of $m_{s t}$, almost entirely eliminating the local resonance bandgap. This behavior is established in the plots for real and imaginary parts of the wavenumber in Figure 4. 6 (b) and (c). For different values of $J$, the 
variation of upper and lower limits of the locally resonant bandgap with mass ratio is shown in Figure 4. 6 (d). The upper and lower limits of the locally resonant bandgap for this case are

$$
\begin{gathered}
\omega_{U}^{P I}=\sqrt{\frac{k_{2} m_{s t}}{m_{1} m_{2}+J m_{s t}}}=\omega_{0} \sqrt{\frac{m_{2} m_{s t}}{m_{1} m_{2}+J m_{s t}}} \\
\omega_{L}^{P I}=\sqrt{\frac{k_{2}}{m_{2}+J}}=\omega_{0} \sqrt{\frac{m_{2}}{m_{2}+J}}
\end{gathered}
$$

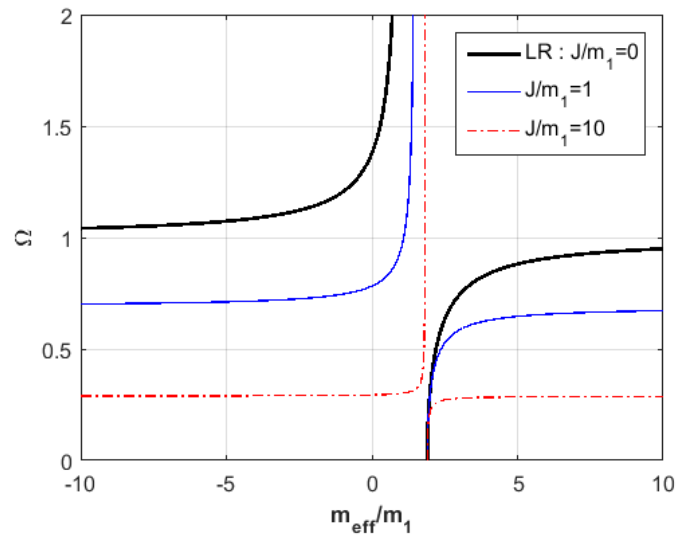

(a)

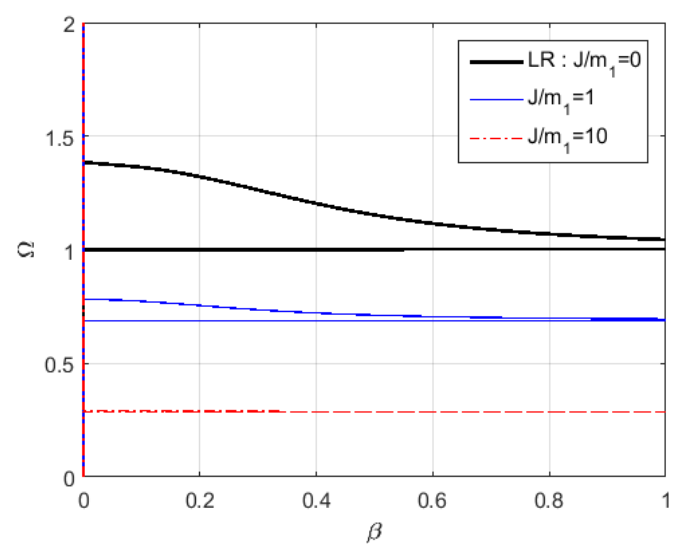

(c)

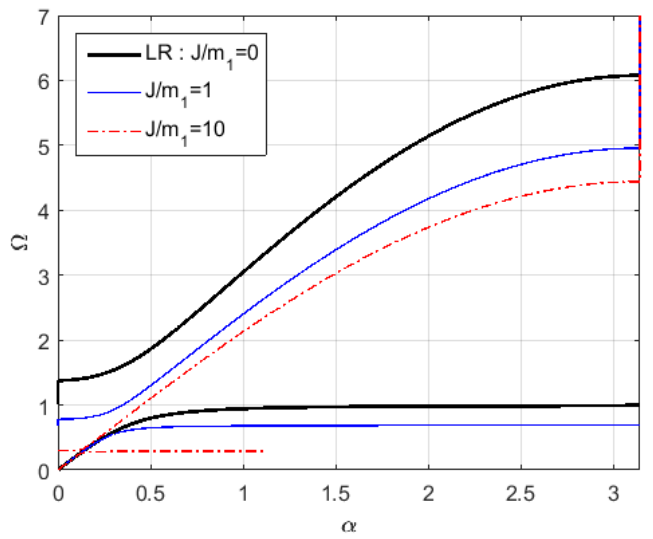

(b)

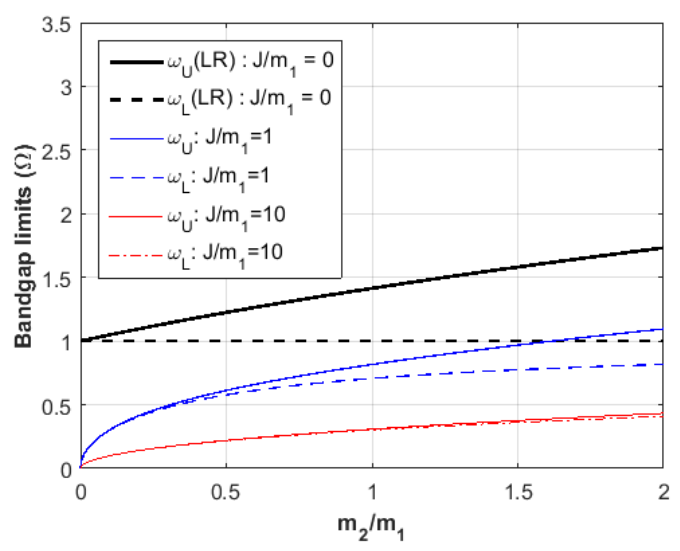

(d)

Figure 4. 6: (a) Normalized effective-mass, (b) real and (c) imaginary parts of the wavenumber versus normalized excitation frequency and (d) mass ratio vs bandgap limits for various values of inertance for acoustic metamaterial with parallel inertant attachment. 
While the lower limit of the locally resonant bandgap $\left(\omega_{L}^{P I}\right)$ is at the shifted local resonance frequency which moves to lower frequencies due to the presence of the inertance $J$, the upper limit, which was $\sqrt{\left(1+m_{2} / m_{1}\right)} \omega_{0}$ for the LRAM is now moved to a lower frequency given by Equation 4.11. It is therefore possible to tune the bandgap to a narrow low-frequency bandwidth by using the parallel inertant attachment without adding a lot of parasitic mass to the structure. Since a high inertance can be achieved with a relatively low device mass, this approach is desirable when targeted low-frequency filtering is required without a high attachment mass.

\subsubsection{Series Inertant Attachment}

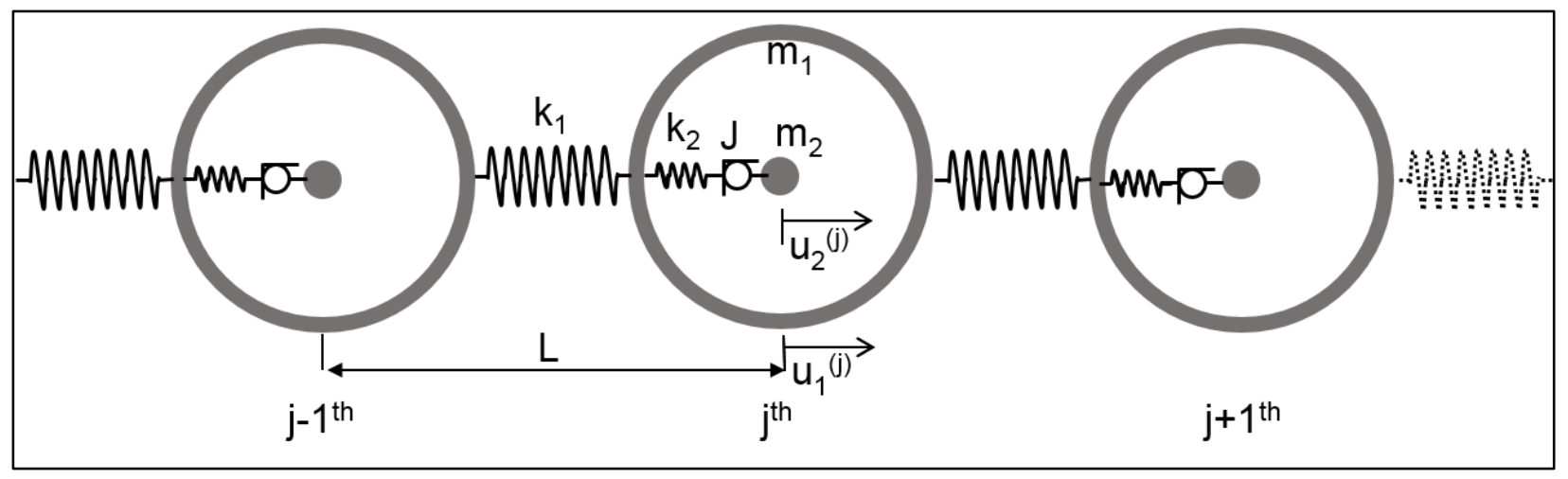

Figure 4. 7: Acoustic metamaterial with local attachments having inerter in series with the

$$
\text { spring. }
$$

The lattice model for an acoustic metamaterial having local attachments with inerter in series with the internal spring is shown in Figure 4. 7. In this case, the limiting behavior is similar to the LRAM for a very high value of $J$ and tends to that of a monatomic lattice with a lattice mass of $m_{1}$ when $J$ becomes very small. The effective-mass plots in Figure 4.8 (a) depict this trend and are given by relation

$$
m_{e f f}^{S I}=\left[m_{1}+\frac{1}{\left(\frac{1}{J}\right)+\left(\frac{1-\Omega^{2}}{m_{2}}\right)}\right]
$$




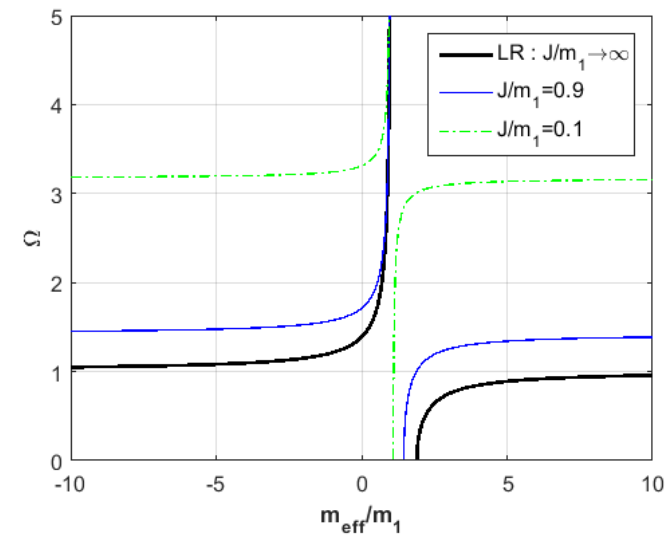

(a)

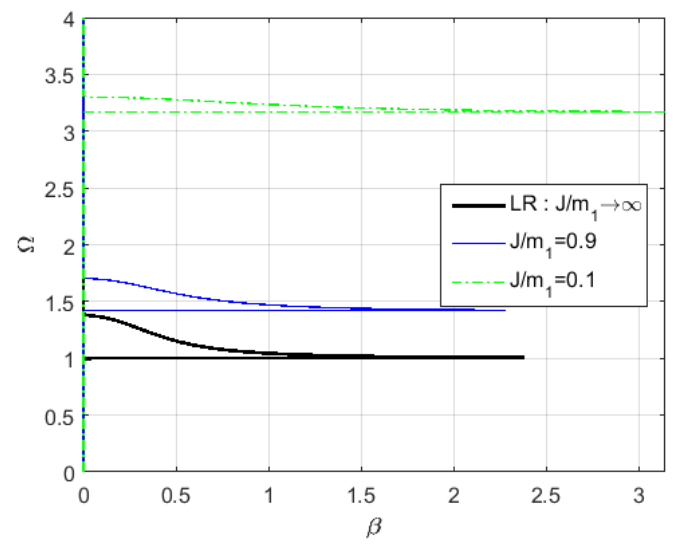

(c)

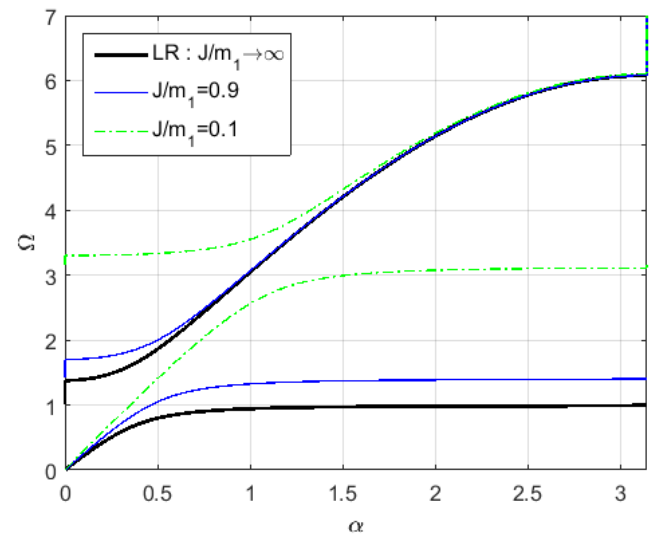

(b)

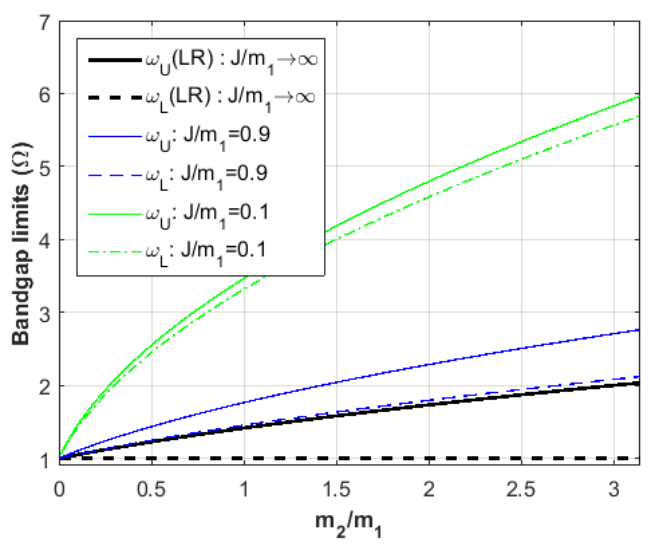

(d)

Figure 4. 8: (a) Normalized effective-mass, (b) real and (c) imaginary parts of the wavenumber versus normalized excitation frequency and (d) mass ratio vs bandgap limits for various values of inertance for acoustic metamaterial with series inertant attachment.

The real and imaginary parts of the wavenumber versus normalized excitation frequency for the series inertant attachment case are shown in Figure 4. 8 (b) and (c). As $J$ tends to a very large value, the dispersion behavior tends to that of the LRAM. With decreasing value of $J$, the bandgap location shifts to higher frequencies, while its extent also diminishes. The upper and lower limits of the locally resonant bandgap for this case are given by 


$$
\begin{aligned}
& \omega_{U}^{S I}=\omega_{0} \sqrt{1+\frac{m_{2}}{m_{1}}+\frac{m_{2}}{J}} \\
& \omega_{L}^{S I}=\omega_{0} \sqrt{1+\frac{m_{2}}{J}}
\end{aligned}
$$

The corresponding mass ratio vs bandgap limits are given in Figure 4. 8 (d). Thus for a very small value of $J$, a relatively narrow bandgap at a higher frequency can be engineered.

Two acoustic metamaterial configurations are explored with inerters in the lattice structure. In the first case, the inerter is present in parallel to the lattice spring while in the second it is present in series. The first is referred to as the parallel inertant lattice (PL) case, while the second is referred to as the series inertant lattice (SL) case. Both cases are considered with locally resonant attachments just as in the LRAM. Therefore, the LRAM acts as the baseline reference for the performance of these cases. Whereas the local attachment is modeled using the effective-mass, an effective stiffness parameter is utilized to capture the effect of the inertance in the lattice. The dispersion behavior for both cases is discussed in the following sections.

\subsubsection{Parallel Inertant Lattice}

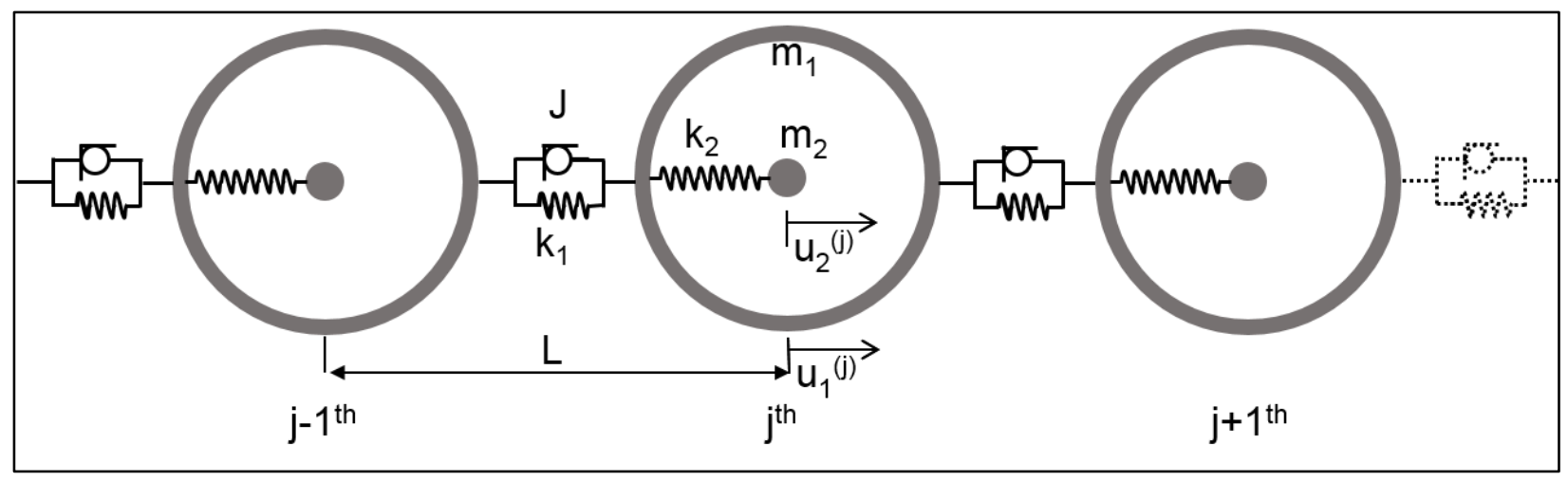

Figure 4. 9: Acoustic metamaterial with lattice structure having inerter in parallel with stiffness.

As can be seen from Figure 4. 9, this case is close to an LRAM with an inerter addition made in the lattice in parallel to the host stiffness, $k_{l}$. To simplify the modeling and follow the 
aforementioned analytical method consistently, the internal feature containing inerter and spring are modeled as an effective stiffness as shown in Figure 4. 9.

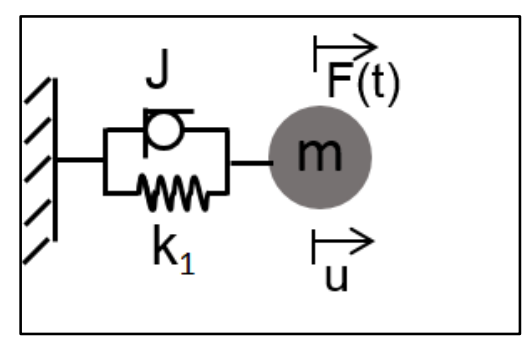

(a)

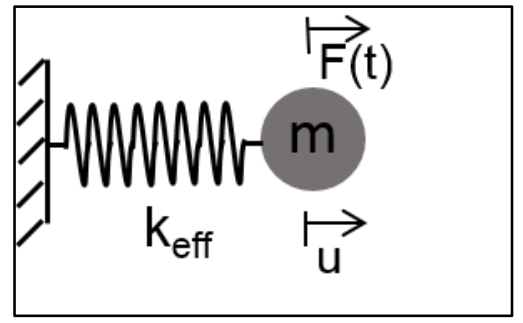

(b)

Figure 4. 10: (a) Parallel inertant SDOF system and (b) its equivalent effective-stiffness model.

By enforcing the displacement response $(u)$ for both models in Figure 4.10 to be the same under a given harmonic force excitation, the effective-stiffness can be derived as

$$
k_{e f f}^{P L}=k_{1}-J \omega^{2}=k_{1}-J \Omega^{2} \omega_{0}^{2}
$$

This effective-stiffness in Equation 4.15 is frequency-dependent and shows that under static loading conditions when $\omega \rightarrow 0$, the stiffness of the lattice is governed by the spring's stiffness, $k_{1}$. It can be noticed from Equation 4.15 that as the excitation frequency is increased for a system with fixed $\mathrm{J}$ and $\mathrm{k}_{1}$, the effective stiffness undergoes softening. By selecting specific parameters, the effective stiffness can be forced to become negative. The added effect of negative mass is studied subsequently. Following the mentioned analytical method yields the dispersion relation in terms of the defining parameters as

$$
q L=\alpha+i \beta=\cos ^{-1}\left[1-\frac{m_{e f f}^{P L} \omega^{2}}{2 k_{e f f}^{P L}}\right]=\cos ^{-1}\left[1-\frac{m_{e f f}^{P L} \Omega^{2} \omega_{0}^{2}}{2 k_{e f f}^{P L}}\right]
$$

where the effective-mass $\left(m_{e f f}^{P L}\right)$ is same as that for the LRAM and is given by Equation 4.1. It is evident from Equation 4.16 that the dispersion relation is dependent not only on effective mass, but also on effective stiffness. It should be noted that traditional LRAM exhibited unusual behavior in the area of negative effective mass. However, due to the presence of an effective 
mass, the behavior of this type of a lattice would be dependent not only on effective mass, but also on effective stiffness. Figure 4. 11 shows the behavior of the normalized effective-stiffness $\left(k_{e f f}^{S L} / k_{1}\right)$ and normalized effective-mass $\left(m_{e f f}^{S L} / m_{1}\right)$ versus normalized excitation frequency for different values of $J$. As $J$ increases, the effective-stiffness becomes negative at a lower excitation frequency. This is directly evident from Equation 4.15. As the effective-mass arises due to the locally resonant attachment, its behavior is not affected by the inertance. Hence, effective-mass of this configuration has the same behavior as the LRAM. The parameter $\left(m_{e f f}^{P L} / k_{e f f}^{P L}\right)$ in Equation 4.16 is representative of the combined effect of effective-stiffness and effective-mass on the bandgap location and degree of attenuation. It should be noted that the degree of attenuation is characterized by the attenuation factor, $\beta$. This parameter is normalized by multiplying it by $\omega_{0}^{2}=k_{2} / m_{2}$ to define an effective-lattice parameter $\left(\omega_{0}^{2} m_{e f f}^{P L} / k_{e f f}^{P L}\right)$ that characterizes the dispersion behavior and is plotted against normalized excitation frequency for $J / m_{1}=1$ and $J / m_{1}=100$ in Figure 4.12 (a) and (b) respectively. The bandgap regions correspond to the frequency ranges in which $m_{e f f}^{P L} / k_{e f f}^{P L}$ is negative when either effective-mass or effective-stiffness alone is negative. From the corresponding plots for the real $(\alpha)$ and imaginary $(\beta)$ parts of the wavenumber $(q L)$ for $J / m_{1}=1$ and $J / m_{1}=100$ in Figure 4.12 (c) and (d) respectively, it can be seen that the double-positive region yields a propagation mode with a positive group velocity $(d \Omega / d q L)$, whereas the double-negative region (for $J / m_{1}=100$ in this case) results in a propagation mode with a negative group velocity, which suggests a regressing wave envelope with a progressing wave phase for narrow-banded harmonic wave packets. It is also interesting to note that the attenuation factor, $\beta$ is unbounded at both the upper and lower bounds of the low-frequency bandgap for the $J / m_{1}=100$ case with a tunable non-zero minima within the bandgap. This behavior indicates that, for an appropriate value of $J$, the degree of attenuation everywhere in the bandgap could be tuned to exceed a desired threshold. 


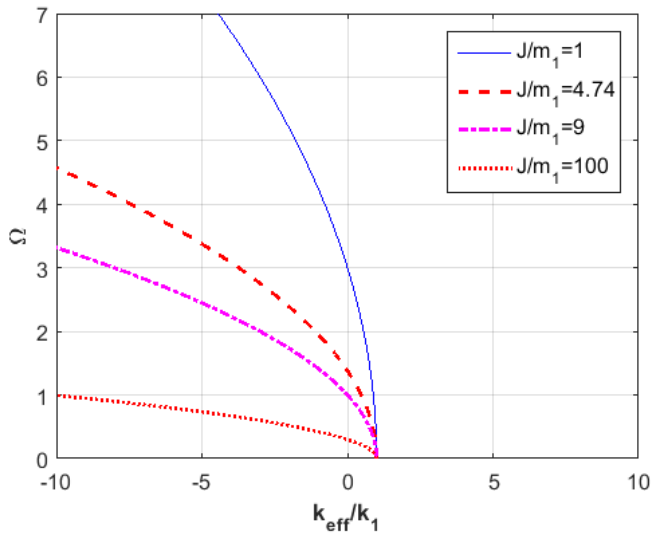

(a)

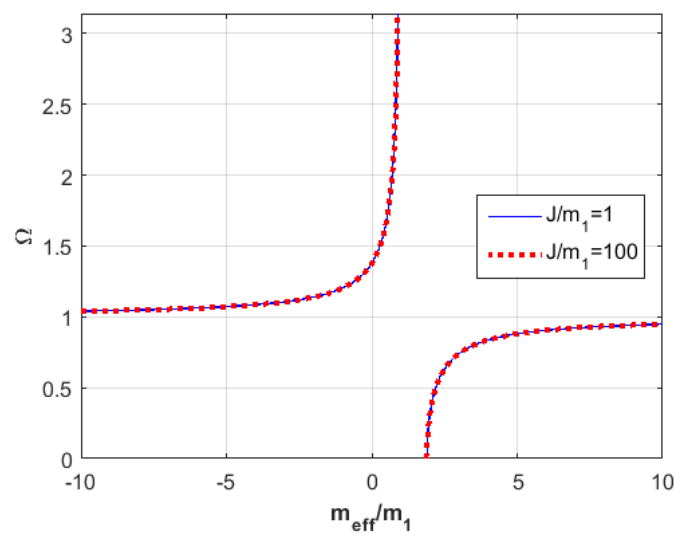

(b)

Figure 4. 11: (a) Normalized effective-stiffness and (b) effective-mass normalized excitation frequency for different values of inertance for the parallel inertant lattice configuration.

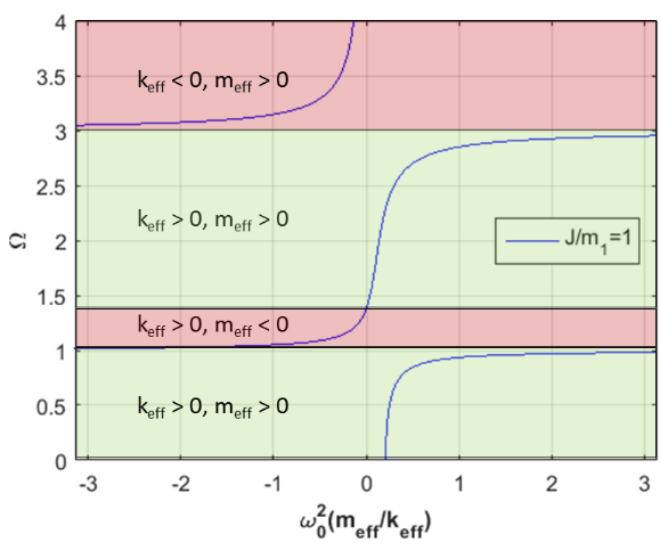

(a)

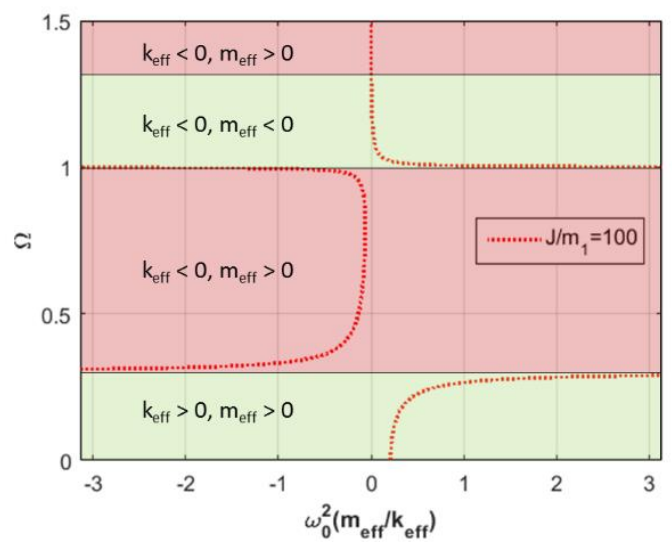

(b) 


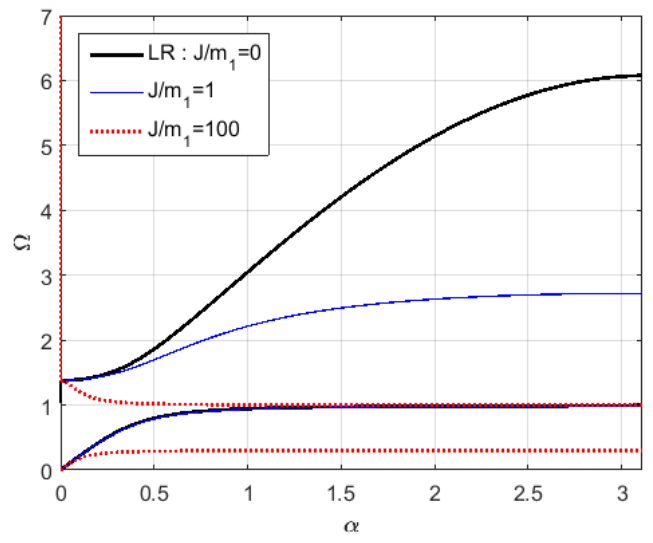

(c)

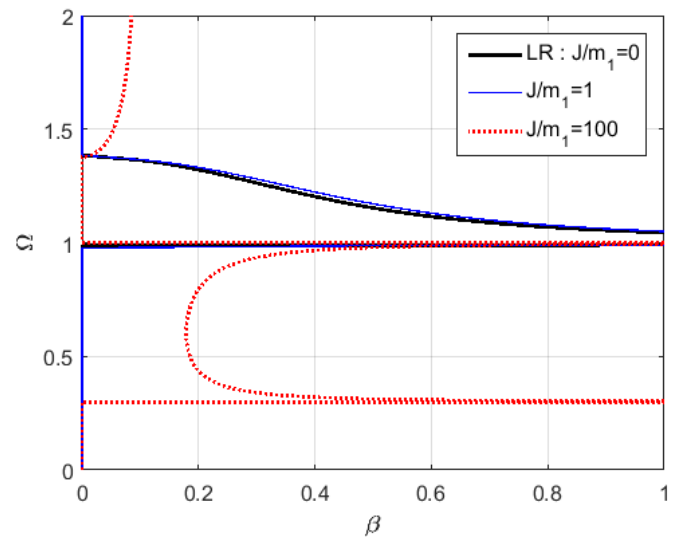

(d)

Figure 4. 12: $\quad$ Normalized effective lattice parameter versus normalized excitation frequency for

(a) $\mathrm{J} / \mathrm{m}_{1}=1$ and (b) $\mathrm{J} / \mathrm{m}_{1}=100$ along with (c) real and (d) imaginary parts of the wavenumber versus normalized excitation frequency for these cases for the parallel inertant lattice configuration.

\subsection{Special Cases}

Two special cases for $J / m_{1}$ are also considered for the parallel inertant lattice as shown in Figure 4. 13 in order to emphasize the tunability of their propagation characteristics in order make the two bandgap frequency regions continuous. These two values of $J / m_{1}$ are obtained by setting the crossover frequency for $k_{e f f}^{P L}$ from positive to negative to coincide with the lower and upper frequency bounds for the bandwidth of effective-mass negativity.

$$
\begin{aligned}
& J_{L B}^{P L}=\left\{J \mid k_{e f f}^{P L}\left(\Omega=\Omega_{L}\right)=0\right\} \\
& J_{U B}^{P L}=\left\{J \mid k_{e f f}^{P L}\left(\Omega=\Omega_{U}\right)=0\right\}
\end{aligned}
$$

where $\Omega_{L}=1$ and $\Omega_{U}=\sqrt{1+\theta_{21}}$ are the lower and upper bounds of the effective-mass negativity for the locally resonant attachment as given by Equation 4.2. Applying the conditions in Equations 4.17 and 4.18, the values of $J$ corresponding to these two special cases for the chosen lattice parameters are obtained as 


$$
\begin{gathered}
J_{L B}^{P L}=\frac{k_{1}}{\omega_{0}^{2}}=90 \\
J_{U B}^{P L}=\frac{k_{1}}{\omega_{0}^{2}\left(1+\theta_{21}\right)}=47.4
\end{gathered}
$$

As $m_{1}=10$, the normalized inertance $J / m_{1}$ for the cases in Equation 4.19 and 4.20 are 9 and 4.74 respectively. The effective-lattice parameter $\left(\omega_{0}^{2} m_{e f f}^{P L} / k_{e f f}^{P L}\right)$ is plotted against the normalized excitation frequency for the two values of $\mathbf{J}$ as shown in Figure 4. 13 and the corresponding plots for real and imaginary parts of the wavenumber are shown in Figure 4. 13. Although the system behaves as a low-pass filter in both the cases, it exhibits distinct propagation. Choosing $J=J_{L B}^{P L}$ gives $J / m_{1}=9$, and since occurrence of $k_{e f f}^{P L}=0$ is tuned to coincide with the lower bound of effective-mass negativity $(\Omega=1)$, a propagation band with double-negativity is obtained immediately above the double-positive propagation band. Once the normalized excitation frequency is above the upper bound of effective-mass negativity, effectivemass turns positive while the effective-stiffness remains negative. This causes the ratio of effective mass and stiffness to remain negative and results in a stop band for all higher frequencies. However, it must be noted that as the excitation frequency becomes very large, the value of the effective lattice parameter $\left(\omega_{0}^{2} m_{e f f}^{P L} / k_{e f f}^{P L}\right)$ asymptotes to zero from the negative side. Therefore, the degree of spatial attenuation for higher frequencies is drastically diminished. For $J / m_{1}=4.74$ where $J=J_{U B}^{P L}$ since occurrence of $k_{e f f}^{P L}=0$ is tuned to coincide with the upper bound of effective-mass negativity $\left(\Omega=\sqrt{1+\theta_{21}}=1.38\right)$, the region of double-negativity is absent. Above the propagation band that corresponds to the double-positive region, first $m_{e f f}^{P L}$ is negative while $k_{e f f}^{P L}$ is positive within the bandgap frequency range for the original LRAM. When excitation is above the upper bound of effective-mass negativity, $k_{e f f}^{P L}$ turns negative while $m_{e f f}^{P L}$ switches to being positive resulting in a continuous stop band. Nonetheless, the degree of spatial attenuation for high frequency excitations is drastically diminished in this case too due to a 
similar asymptotic behavior as before. For obtaining a continuous higher frequency bandgap, $J=J_{U B}^{P L}$ and $J=J_{L B}^{P L}$ are the values of the inertance that provide the lowest and highest cut-off frequencies respectively. The PL configuration could thus enable low-pass acoustic filters with possibility of propagation modes having negative group velocity.

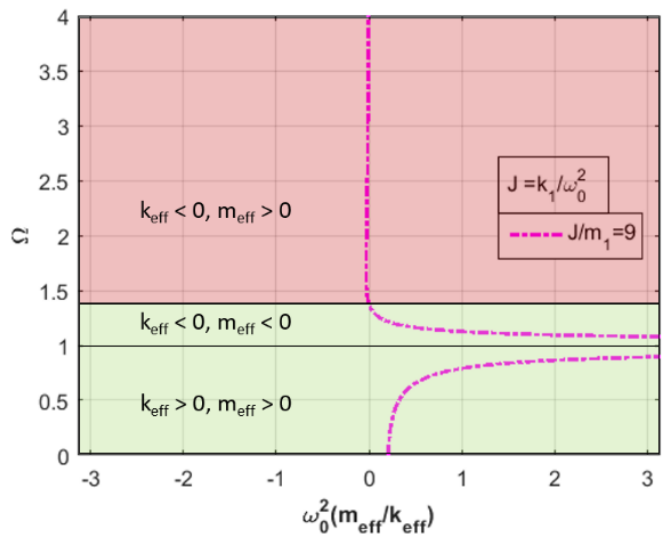

(a)

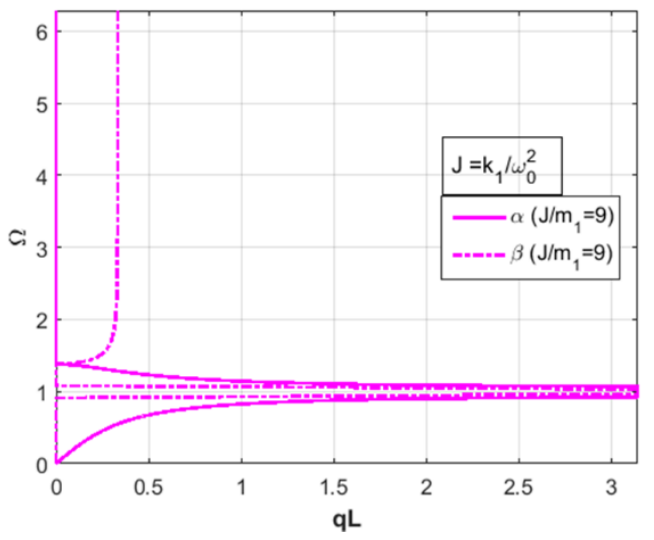

(c)

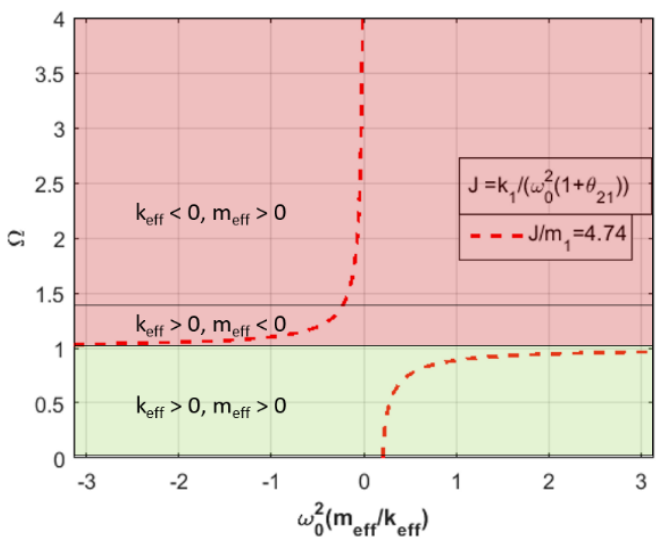

(b)

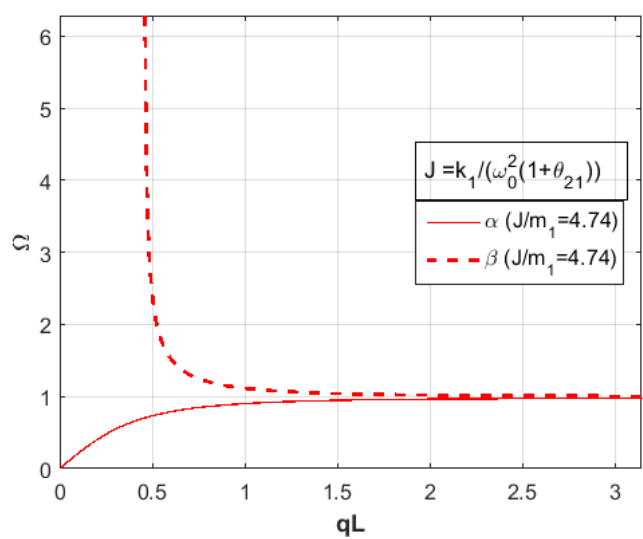

(d)

Figure 4. 13: Normalized effective lattice parameter versus normalized excitation frequency for

(a) $\mathrm{J} / \mathrm{m}_{1}=9$ and (b) $\mathrm{J} / \mathrm{m}_{1}=4.74$ along with (c) real and (d) imaginary parts of the wavenumber versus normalized excitation frequency for these cases for the parallel inertant lattice configuration. 


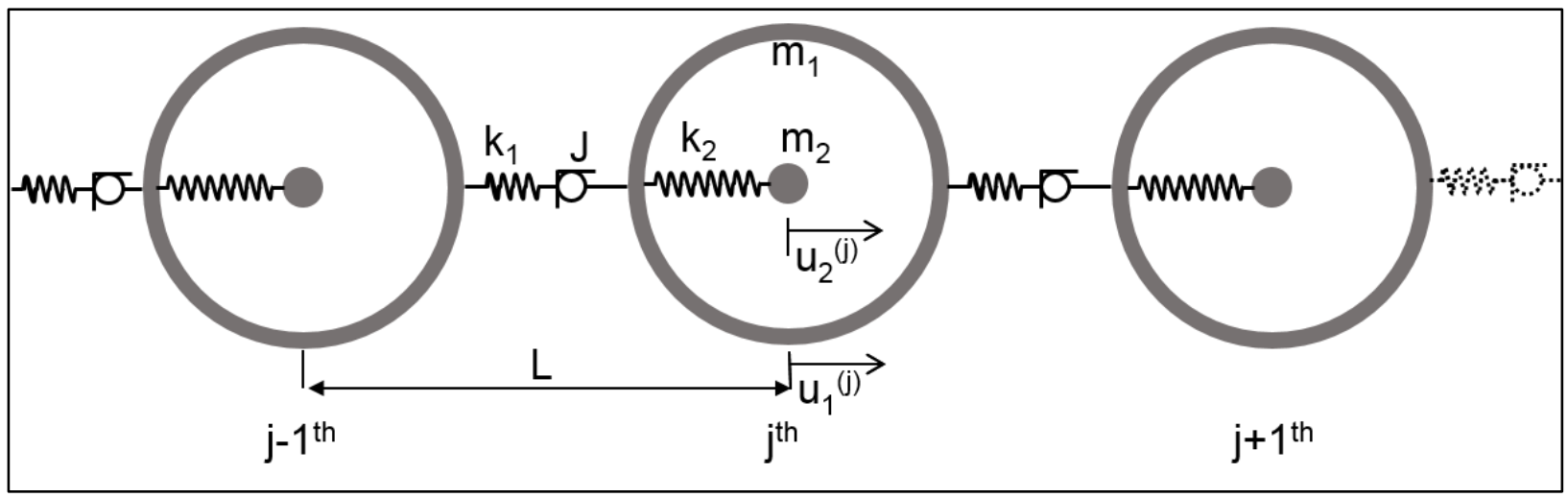

Figure 4. 14: Acoustic metamaterial with lattice structure having inerter in series with stiffness.

The second configuration with inerter in the lattice that is considered is the series inertant lattice (SL) case shown in Figure 4. 14. The inerter, in this case, is in series connection with the spring in the lattice. Similar to the PL case, locally resonant attachments are retained and hence the effective mass is given by the same expression (Equation 4.1). The effective stiffness is computed for this case as well, resulting in the expression given in Equation 4.21. The effective-stiffness for the series inertant lattice case is obtained as

$$
k_{e f f}^{S L}=\frac{k_{1}}{1-\frac{k_{1}}{J \omega^{2}}}=\frac{k_{1}}{1-\frac{k_{1}}{J \Omega^{2} \omega_{0}^{2}}}
$$

The normalized effective-stiffness, $k_{e f f}^{S L} / k_{1}$ and normalized effective-mass, $m_{e f f}^{S L} / m_{1}$ are plotted against normalized excitation frequency $(\Omega)$ for different values of $J$ in Figure 4. 15. As suggested by Equation 4.21, the effective-stiffness becomes unbounded at $\Omega=\sqrt{k_{1} /\left(J \omega_{0}^{2}\right)}$. For excitation frequencies below this value, the effective-stiffness is negative while it is positive above. It asymptotes to $k_{e f f}^{S L} / k_{1}=1$ for a very high excitation frequency. The combination of effective stiffness and mass as seen in Figure 4. 16 suggests an ultra-low frequency bandgap. Dispersion behavior for a low $\left(J / m_{1}=1\right)$ and higher $\left(J / m_{1}=20\right)$ value of inertance are discussed first. Plots for the effective lattice parameter $\left(\omega_{0}^{2} m_{e f f}^{P L} / k_{e f f}^{P L}\right)$ and real and imaginary parts of the 
wavenumber versus normalized excitation frequency for these two values of $J / m_{1}$ are shown in Figure 4. 16. In both cases, two disjoint bandgap regions are obtained among which one extends from the long wavelength limit to a first cut-on frequency. The location of this cut-on frequency and nature of the pass bands are dependent on the frequency at which $k_{e f f}^{P L}$ transitions from a negative to positive value. If this transition lies above (as for $J / m_{1}=1$ ) the local resonance frequency of the attachments, a double-negative pass band is interspersed between the two stop bands while if it is below (as for $J / m_{1}=20$ ), both pass bands are of the double-positive type as is borne out in Figure 4.16 (c) and (d). The effective lattice parameter asymptotes to $\omega_{0}^{2} m_{1} / k_{1}=$ 0.11 as the excitation frequency becomes very large. This ensures the upper double-positive propagation band extends to higher frequencies until the lattice discretization bandgap is encountered.

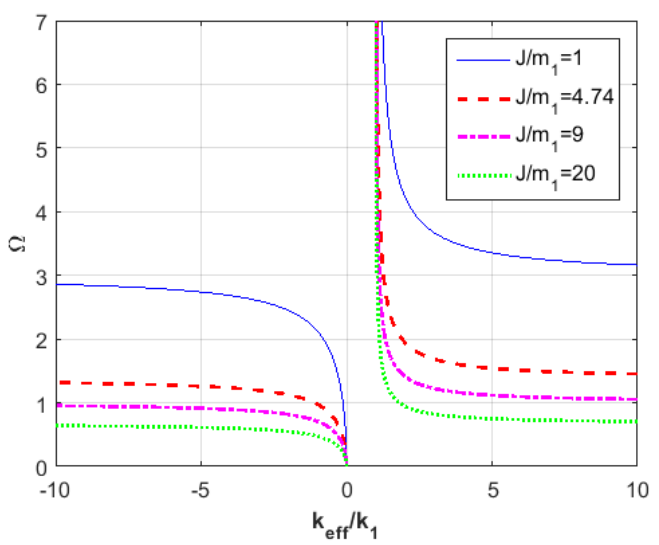

(a)

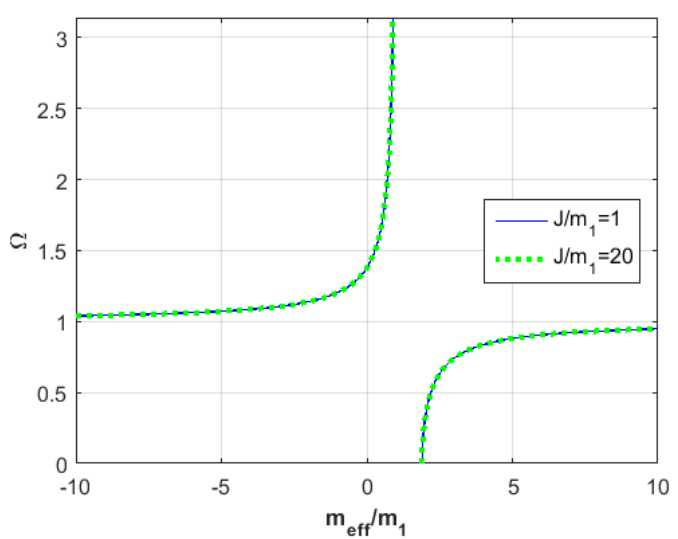

(b)

Figure 4. 15: (a) Normalized effective-stiffness and (b) effective-mass versus normalized excitation frequency for different values of inertance for the series inertant lattice configuration. 


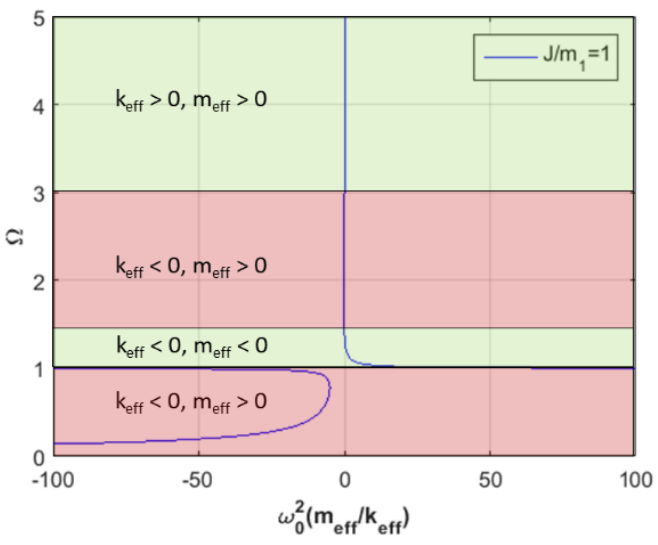

(a)

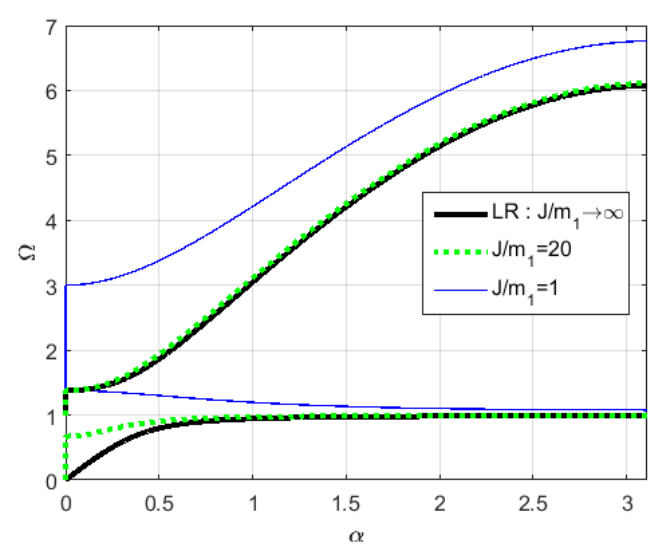

(c)

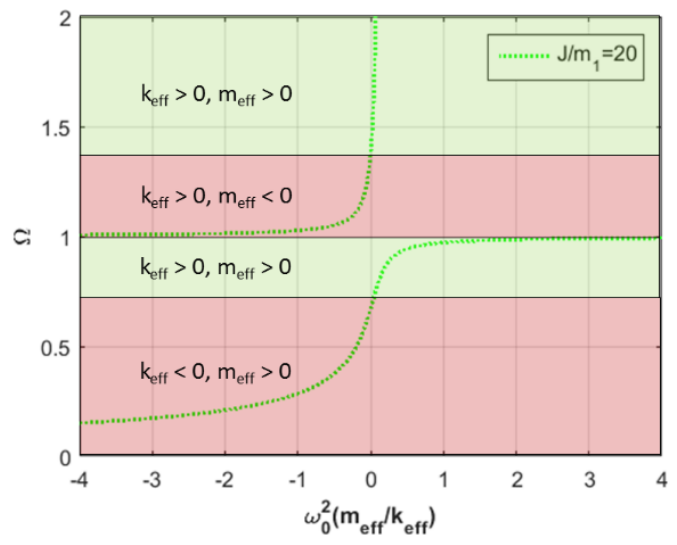

(b)

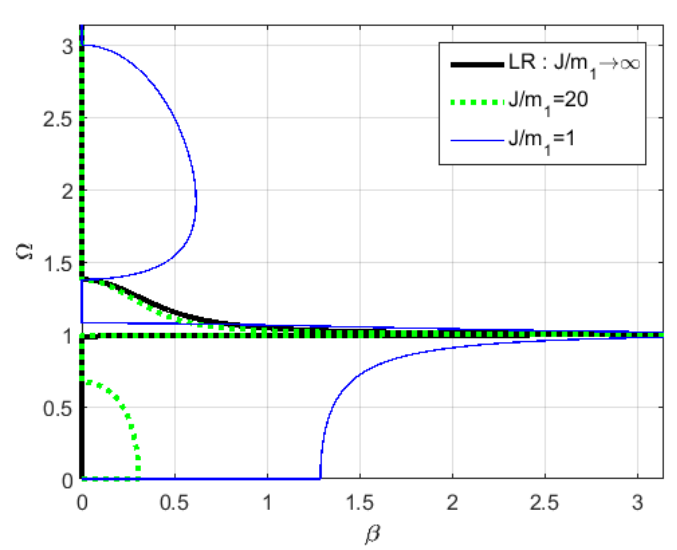

(d)

Figure 4. 16: Normalized effective lattice parameter versus normalized excitation frequency for (a) $\mathrm{J} / \mathrm{m}_{1}=1$ and (b) $\mathrm{J} / \mathrm{m}_{1}=20$ along with (c) real and (d) imaginary parts of the wavenumber versus normalized excitation frequency for these cases for the series inertant lattice configuration.

\subsection{Special Cases}

It can be stated that by choosing specific $\mathrm{J}$ for the present case, the ultra-low frequency band gap can be expanded by joining adjacent regions as seen in Figure 4. 17. Similar to the previous case, the same conditions are applied for the SL to obtain values of $J$ for limiting cut-on frequencies for the ultra-low frequency bandgap. The same values of $\mathbf{J}$ as the PL case are obtained. The cut-on frequency for the contiguous ultra-low frequency bandgap is highest $\left(\Omega_{c o}=\sqrt{1+\theta_{21}}\right)$ for 
$J / m_{1}=9$ (i.e. $J=J_{L B}^{S L}$ ), and lowest $\left(\Omega_{c o}=1\right.$ ) for $J / m_{1}=4.74$ (i.e. $J=J_{U B}^{S L}$ ), as can be noted from the plots for the effective lattice parameter versus normalized excitation frequency in Figure 4. 17 (a) and (b).

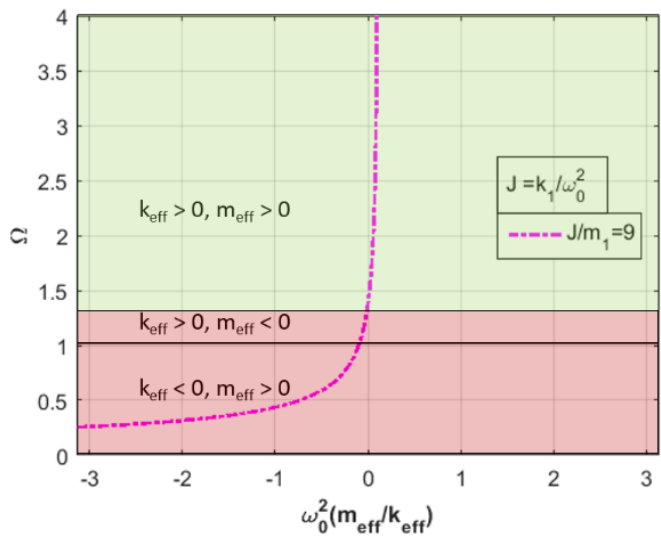

(a)

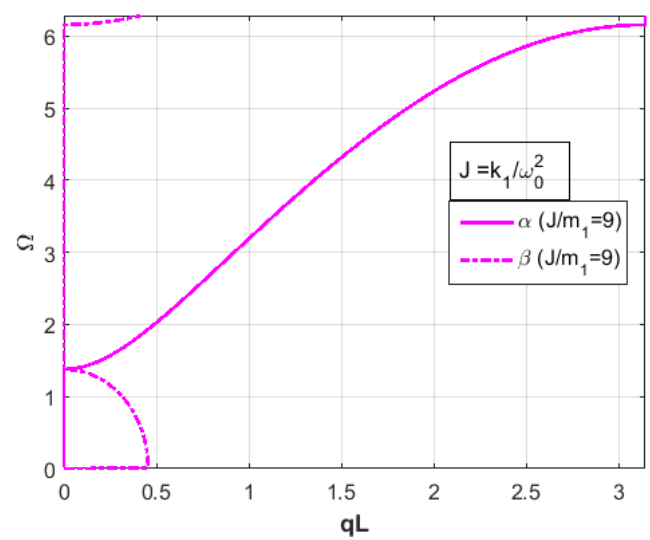

(c)

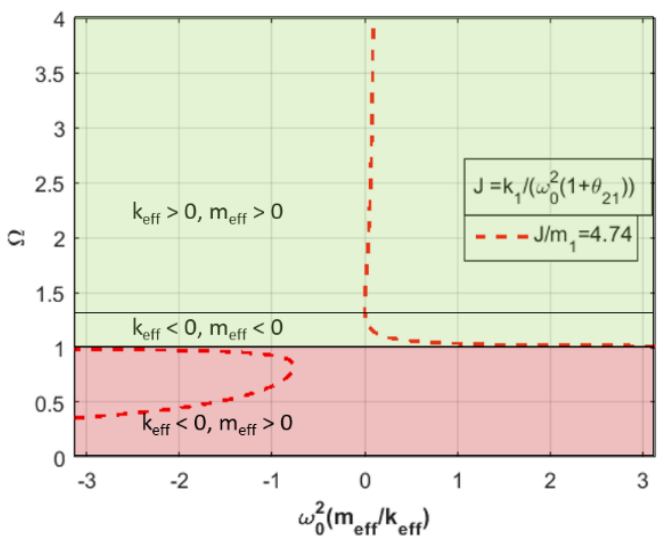

(b)

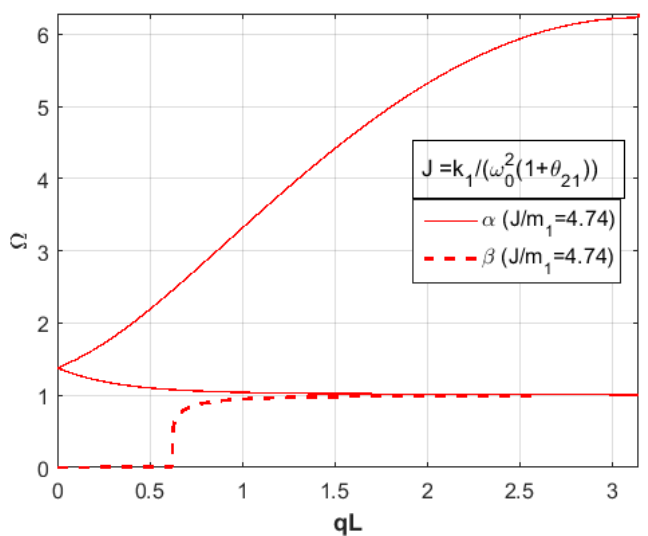

(d)

Figure 4. 17: Normalized effective lattice parameter versus normalized excitation frequency for

(a) $\mathrm{J} / \mathrm{m}_{1}=9$ and (b) $\mathrm{J} / \mathrm{m}_{1}=4.74$ along with (c) real and (d) imaginary parts of the wavenumber versus normalized excitation frequency for these cases for the series inertant lattice configuration.

The dispersion behavior for these values of $J$ are shown in Figure 4.17 (c) and (d). Although the bandgap width is greater for the $J / m_{1}=9$ case, the attenuation factor, $\beta$ remains bounded and 
relatively small over the entire bandgap width when compared to the $J / m_{1}=4.74$ case for which $\beta$ tends to become unbounded at the cut-on frequency. The propagation region above the bandgap for the $J / m_{1}=4.74$ case consists of two distinct modes due to the presence of a double-negative region immediately above the cut-on frequency. This presents the possibility of employing SL configuration to enable ultra-low-frequency filtering devices.

\subsection{Device Implications}

The dispersion characteristic for acoustic metamaterial configurations with inerters in the local attachment or in the lattice could provide a means of enriching the tunable bandgap behavior obtained in LRAMs without the addition of large local attachment mass. Thus inertant acoustic metamaterials show promise of overcoming limitations of mass ratio encountered in LRAMs. This could have implications for realizing devices such as tunable narrow-banded low-frequency acoustic filters and high-pass filters with ultra-low frequency bandgaps that cover the long wavelength limit. Low-pass filters with the possibility of propagation modes having negative group velocity may be possible too.

Using combinations of the inertant acoustic metamaterial configurations is considered in this study. Further, devices having unusual dynamic characteristics could be postulated. For example, the device implications of cascading the parallel inertant lattice (PL) configuration and the series inertant lattice (SL) configuration in succession is considered. With reference to the discussion in Section 4.2.5, consider a PL configuration with $J / m_{1}=4.74$ and an SL configuration with $J / m_{1}=9$ that are connected in sequence. The PL behaves as a low-pass filter attenuating harmonic waves with frequency above the local resonance frequency of attached mass and the SL as a high-pass filter attenuating harmonic waves with frequencies below $\Omega=\sqrt{1+\theta_{21}}=1.378$. This results in an overlap in their bandgap frequency ranges. As can be seen from the plots in Figure 4. 18 (a) for the attenuation factor, $(\beta)$ for PL and SL, the overall stop band for this device covers all excitation frequencies leading to it act as a complete longitudinal elastic wave inhibitor. 
Choosing $\mathrm{J} / \mathrm{m}_{1}=4.74$ for both PL and SL results in the low and high frequency bandgaps becoming continuous at $\Omega=1$ where $\beta$ is also unbounded for the two configurations. By exploring new combinations of inertant metamaterial configurations and tuning the inertance in the various sub-structures, the degree of attenuation over the entire frequency range may be optimized. Also, more interesting characteristics for specialized applications may be realized. The ability to realize such inertant acoustic metamaterials with omnipresent bandgap may result in designing materials with the ability to absorb mechanical vibrations and shock.

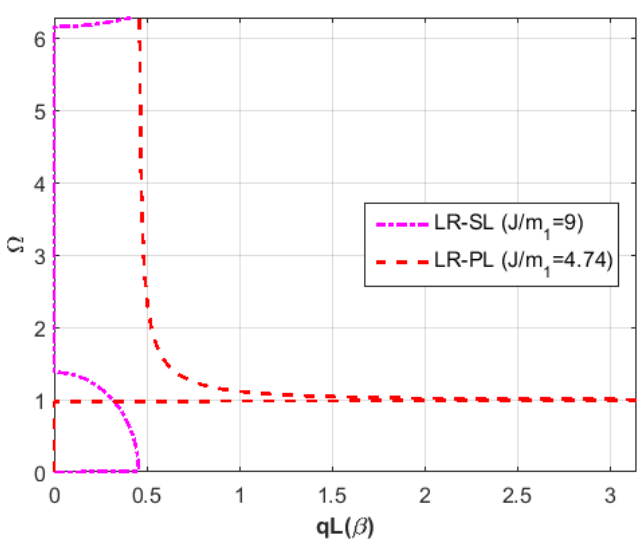

(a)

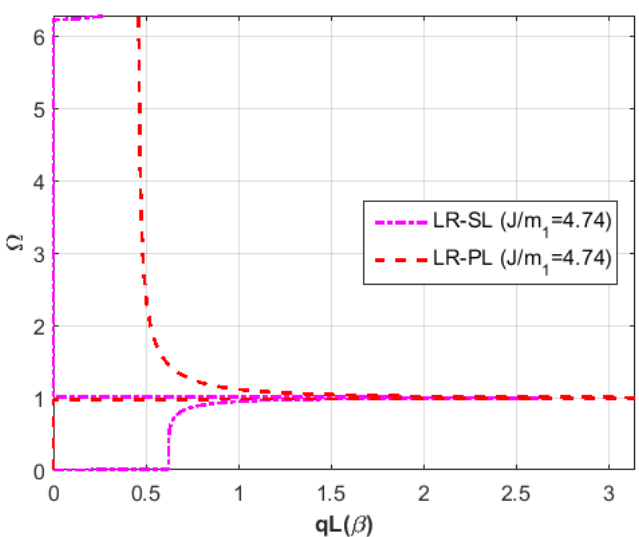

(b)

Figure 4. 18: Attenuation factors for the longitudinal wave inhibitor with combined parallel and series inertant lattice configurations having (a) $\mathrm{J} / \mathrm{m}_{1}=4.74$ for $P L$ and $\mathrm{J} / \mathrm{m}_{1}=9$ for $S L$ and $(b)$ $J / m_{1}=4.74$ for both $P L$ and $S L$.

A comparison table displaying the major characteristics of all the cases taken under consideration are presented as follows. 
Table 4. 1: Key propagation characteristics of various inertant configurations.

\begin{tabular}{|c|c|c|c|c|c|c|c|}
\hline \multirow[b]{2}{*}{ Characteristics } & \multirow{2}{*}{$\begin{array}{c}\text { No Inerter } \\
\text { LRAM }\end{array}$} & \multicolumn{3}{|c|}{ Inerter In Local Attachment } & \multicolumn{3}{|c|}{ Locally Resonant, Inerter In Lattice } \\
\hline & & $\begin{array}{l}\text { Wholly } \\
\text { Inertant }\end{array}$ & $\begin{array}{l}\text { Parallel } \\
\text { Inertant }\end{array}$ & $\begin{array}{c}\text { Series } \\
\text { Inertant }\end{array}$ & $\begin{array}{l}\text { Parallel } \\
\text { Inertant }\end{array}$ & $\begin{array}{c}\text { Series } \\
\text { Inertant }\end{array}$ & Combination $^{\mathrm{a}}$ \\
\hline$m_{e f f}<0$ & $\checkmark$ & $\mathrm{x}$ & $\checkmark$ & $\checkmark$ & $\checkmark$ & $\checkmark$ & $\checkmark$ \\
\hline$k_{e f f}<0$ & $\mathrm{x}$ & $\mathrm{x}$ & $\mathrm{x}$ & $\mathrm{x}$ & $\checkmark$ & $\checkmark$ & $\checkmark$ \\
\hline$v_{g}{ }^{\mathrm{b}}<0$ & $\mathrm{x}$ & $\mathrm{X}$ & $\mathrm{x}$ & $\mathrm{x}$ & $\bar{\checkmark}$ & $\mathrm{x}$ & $\checkmark$ \\
\hline $\begin{array}{c}\text { Multiple } \\
\text { unbounded } \beta \\
\text { locations }\end{array}$ & $\mathrm{x}$ & $\mathrm{x}$ & $\mathrm{x}$ & $\mathrm{X}$ & $\checkmark$ & $\checkmark$ & $\checkmark$ \\
\hline $\begin{array}{c}\mathrm{BR}^{\mathrm{c}}, \mathrm{LP}^{\mathrm{c}}, \mathrm{HP}^{\mathrm{d}}, \\
\mathrm{ULF}^{\mathrm{e}}\end{array}$ & BR & LP & LP, BR & BR & LP, BR & $\begin{array}{c}\text { HP, ULF, } \\
\text { BR }\end{array}$ & $\begin{array}{c}\text { LP, HP, ULF, } \\
\text { BR, No } \\
\text { Propagation }\end{array}$ \\
\hline
\end{tabular}

${ }^{\mathrm{a}}$ Combination of Series and parallel lattice configurations cascaded in series.

${ }^{\mathrm{b}} v_{g}$ is group velocity.

${ }^{\mathrm{c}}$ BR Band Rejection

${ }^{\mathrm{d}}$ LP Low Pass Filter

${ }^{\mathrm{e}} \mathrm{HP}$ High Pass Filter

${ }^{\mathrm{f}}$ ULF Ultra Low-Frequency Filter

\subsection{Summary}

The longitudinal elastic wave propagation characteristics of inertant acoustic metamaterial configurations having inerters either in the local attachments or in the lattice were investigated using effective models for their discrete element lattice representations. Inerters, which are mechanical elements that provide a force response proportional to the relative acceleration across them, have been shown to have relatively low device mass in comparison to the dynamic mass presence they display due to their inertance. While the presence of inerters in the local 
attachments was modeled using an effective-mass for the lattice, the presence of inerters in the lattice was modeled using an effective-stiffness. This approach provides a simple and efficient means to characterize the behavior of inertant acoustic metamaterials relative to the LRAM.

Three configurations involving inerters in the local attachment were considered. For the wholly inertant local attachment case, it was found that presence of the inerter provided a means to alter the dynamic mass participation in the lattice. When implemented in lattice structures, tuning the value of the inertance alters the cut-off frequency for the discretization bandgap thereby changing its behavior as a low-pass filter. By employing parallel inertant attachments, it was found that narrow-banded selective low-frequency filtering can be achieved without the need for a bulky local mass addition. This could enable targeting specific low frequencies for removal from a wideband incoming wave. On the other hand, a configuration with inerter attached in series to the internal spring assigns the bandgap to a narrower band at a higher frequency compared to the locally resonant case when inertance is small. whereas its behavior tends to the locally resonant case for larger inertance.

Two configurations having inerters in the lattice along with purely resonant local attachments were also considered. The presence of the inerter in the lattice was modeled using an effective stiffness, while the effective-mass due to the attachment remained the same as that for the locally resonant case. It was found that the location, extent of the bandgaps and the propagation characteristics were dependent on an effective lattice parameter, which was proportional to the ratio of the effective-mass to the effective-stiffness. Negative and extreme stiffness was found to be attainable for these configurations depending on the frequency of excitation. In the case of the parallel inertant lattice, it behaves like a low-pass filter if tuned values of inertance are used to produce a continuous higher frequency bandgap. The low-frequency propagation mode in the frequency range where both effective-mass and effective-stiffness are negative displays a negative group velocity. In the case of the series inertant lattice, tuning the inertance could create a high-pass filter with an ultra-low frequency bandgap covering the long wavelength limit 
without the use of grounded elements. A propagation mode with negative group velocity can also be obtained in this case by engineering a double-negative region within the high-pass region.

Aforementioned device characteristics for the standalone inertant acoustic metamaterial configurations that have been considered in this study. In addition, combinations of different inertant configurations with tuned inertance may be employed to further enrich the dynamic characteristics. For instance, deploying a tuned parallel inertant lattice configuration in succession with a series inertant lattice configuration could create a complete longitudinal elastic wave inhibitor that attenuates the entire range of frequencies of interest. Practical and scalable designs for inerters with low device mass and precise response could thus enable the realization of extremely broadband vibration and shock isolators using this approach. There is a possibility of attaining particularly designed dispersion characteristics which are uniquely suited to specific scenarios. By exploring optimized and multi-dimensional combinations of acoustic metamaterial designs with inerters motivates their further research and development. Their potentially transformative applications include mechanical wave manipulators, protective structures, transducers and multifunctional structures. 


\section{CHAPTER V \\ CONCLUSIONS AND RECOMMENDATIONS}

\subsection{Conclusions}

The rapid pace of modernization in the recent past has laid great emphasis on adaptive materials. Engineering new materials has transcended to the structural level, giving rise to metamaterials. The properties displayed by such materials are unique to their configurations and can display behavior that are not readily available in natural materials. The most widely explored acoustic metamaterials are the locally resonant type, which employ periodic resonating attachments in order to impart wave-insulating capability designed for certain frequencies. These and many other AM employ local resonance or instabilities in local features. However, the capabilities of AM to act as wave manipulators can be enhanced by the introduction of new local configurations that display varied dynamic phenomena.

In this context, AM configurations with nonlinear and inertant inclusions within host structures have been explored. Discrete mass-spring lumped parameter models based on effective mass and stiffness were used for analytical and numerical studies in order to obtain dispersion characteristics of these AM. Further, numerical and experimental demonstrations of device implications stemming from these studies were accomplished. A summary of major contributions is as follows.

A perturbation technique was applied to an infinite 1-D lattice with cubic nonlinear local attachments and shifts in dispersion curves was observed. The dispersion curves indicate that hardening type cubic nonlinearity causes the lower bound of the bandgap to shift upwards. 
Simulation study performed in Abaqus, by modeling a discrete mass-spring system demonstrated amplitude-dependent behavior introduced due to the local nonlinearity. Bandgap shifts as predicted by the perturbation technique was demonstrated through the simulations. This suggested the capability of the NLAM to act as an amplitude dependent acoustic filter. Further, the ability of NLAM to alter the frequency content of a wave traversing through it was observed through simulations. It was observed that the frequency content of the transmitted wave was at a lower frequency compared to that of the incident wave. Based on this frequency shift phenomenon and combining it with the bandgap of an LRAM, a direction-biased waveguide device was devised. The direction bias effect was demonstrated through simulations using finite 1-D mass-spring chains.

Based on the numerical study, a test-article consisting of LRAM and NLAM counterparts is manufactured to verify the direction-bias effect through experiments. The test-article was fabricated by employing periodically attached cantilever beams with a specifically modified geometry to realize the required nonlinearity through contact. A numerical routine to generate the root profile to obtain predefined nonlinear stiffness response was developed in Matlab. The NLAM part of the test-article faced significant manufacturing challenges, which resulted in resorting to a hybrid fabrication process that involved milling, waterjet cutting, and 3-D printing. The characteristic band gap of LRAM and NLAM were experimentally obtained and compared with theoretical predictions. The bandgaps of the test-articles displayed apparent deviations from theory, due to the intricacies involved with design and realization of a pre-defined nonlinearity.

Experimental testing of the assembled test-article revealed an interesting feature in the vicinity of $1080 \mathrm{~Hz}$, wherein amplitude, and frequency dependent transmission was observed to be coherent with the simulations. Although at a lower frequency, this is an indication of direction-bias behavior. Further examination of the transmissibility curves demonstrated minor direction bias in the designed frequency range. 
In addition to nonlinear attachments, inertant configurations were investigated and theoretically obtained dispersion relations were presented. Interesting wave manipulation phenomena were observed and device implications based on these findings have been made. Of the various possible devices, the most interesting was the series inertant lattice (SL) configuration which provides promising wave filtering capacity at ultra-low frequencies. By combining a low-pass and a high-pass filtering device, a resultant configuration was proposed which shows the potential to act as an insulator for a very large frequency band.

\subsection{Recommendations}

The experimental setup and methods involved in this study were designed with the objective of capturing direction-bias effect in longitudinal elastic waves using nonlinear stiffness in periodic local attachments. Nonlinearity in itself has been dreaded in history for being unpredictable and chaotic. In experiments that deal with such behavior, the need to devise a systematic setup and specific experimental techniques to capture the behavior cannot be exaggerated.

The method adopted in this study, to realize nonlinearity involves physical contact, which may lead to higher order phenomena such as local wavelet generations. This may interfere with the required effect and thus, systems deriving nonlinearity from alternative methods such as material nonlinearity need to be explored. If the experimental setup is designed based on the assumed 1-D approximations, it could create an opportunity to study only the phenomenon of interest and discard any interrupting effects which complicate data analysis. Although the exhaustive development of experimental setup and instrumentation improve the capability to test nonlinear interactions, the improvement in modeling techniques is important. Lumped parameter model studies were used to establish the direction-bias effect and subsequent experimental setup was designed. To this end, there is a scope to employ much robust simulation methods which consider higher order effects and go beyond 1-D approximations. The present study focusses on the application of only NLH type nonlinearity. However, there are various other types of 
nonlinearities such as - softening, a combination of hardening and softening, and quadratic nonlinearities. These can be combined in various different configurations and explored further.

The study on inertant acoustic metamaterials, on the other hand, is in the early stages of inception and there is definitely scope to build on the lines of simulations to study the many frequencydependent phenomena. The present study is limited to the conception of inertant AM through lumped parameter models, however, the viability of realizing these devices for a real-world scenario can add value to this research area. This can be augmented by the capabilities of advanced manufacturing technologies such as additive manufacturing, self-assembling structures, and MEMS. Another possibility may be, to study the combined effects of inerters and nonlinear inclusions. The combinations explored in this study were limited to two sections LRAM/NLAM and SL/PL, however, devices consisting of multiple units can be investigated in order to obtain effects that depend on the hierarchy of assembly. The ability to investigate such intricacies of the field will definitely open up a wide range of possible applications that are not yet within the bounds of imagination. 


\section{REFERENCES}

[1] Z. Hashin, "Analysis of Composite Materials-A Survey," Journal of Applied Mechanics, vol. 50, pp. 481-505, 1983.

[2] W. S. Weiglhofer, Introduction to complex mediums for optics and electromagnetics vol. 123: SPIE press, 2003.

[3] W. Thomson, "XLVI. On the reflexion and refraction of light," Philosophical Magazine Series 5, vol. 26, pp. 414-425, 1888.

[4] G. V. Viktor, "The electrodynamics of substances with simultaneously negative values of $\varepsilon$ and $\mu, "$ Phys. Usp., vol. 10, p. 509, 1968.

[5] J. B. Pendry, "Negative Refraction Makes a Perfect Lens," Physical Review Letters, vol. 85, pp. 3966-3969, 2000.

[6] R. A. Shelby, D. R. Smith, and S. Schultz, "Experimental Verification of a Negative Index of Refraction," Science, vol. 292, pp. 77-79, 2001.

[7] J. B. Pendry, D. Schurig, and D. R. Smith, "Controlling Electromagnetic Fields," Science, vol. 312, pp. 1780-1782, 2006.

[8] D. Schurig, J. J. Mock, B. J. Justice, S. A. Cummer, J. B. Pendry, A. F. Starr, et al., "Metamaterial Electromagnetic Cloak at Microwave Frequencies," Science, vol. 314, pp. 977-980, 2006.

[9] R. Martinez-Sala, J. Sancho, J. V. Sanchez, V. Gomez, J. Llinares, and F. Meseguer, "Sound attenuation by sculpture," Nature, vol. 378, pp. 241-241, 1995. 
[10] J. H. Vincent, "LX. On the construction of a mechanical model to illustrate Helmholtz's theory of dispersion," Philosophical Magazine, vol. 46, pp. 557-563, 1898.

[11] Z. Liu, X. Zhang, Y. Mao, Y. Y. Zhu, Z. Yang, C. T. Chan, et al., "Locally Resonant Sonic Materials," Science, vol. 289, pp. 1734-1736, 2000.

[12] H. H. Huang, C. T. Sun, and G. L. Huang, "On the negative effective mass density in acoustic metamaterials," International Journal of Engineering Science, vol. 47, pp. 610-617, 2009.

[13] C. Goffaux, J. Sánchez-Dehesa, A. L. Yeyati, P. Lambin, A. Khelif, J. O. Vasseur, et al., "Evidence of Fano-Like Interference Phenomena in Locally Resonant Materials," Physical Review Letters, vol. 88, p. 225502, 2002.

[14] R. Sainidou, B. Djafari-Rouhani, Y. Pennec, and J. O. Vasseur, "Locally resonant phononic crystals made of hollow spheres or cylinders," Physical Review B, vol. 73, p. 024302, 2006.

[15] G. Wang, X. Wen, J. Wen, L. Shao, and Y. Liu, "Two-Dimensional Locally Resonant Phononic Crystals with Binary Structures," Physical Review Letters, vol. 93, p. 154302, 2004.

[16] Y. Xiao, J. Wen, G. Wang, and X. Wen, "Theoretical and Experimental Study of Locally Resonant and Bragg Band Gaps in Flexural Beams Carrying Periodic Arrays of Beam-Like Resonators," Journal of Vibration and Acoustics, vol. 135, pp. 041006-041006, 2013. 
[17] T. L. Smith, K. Rao, and I. Dyer, "Attenuation of plate flexural waves by a layer of dynamic absorbers," The National Academies of Sciences, Engineering, Medicine, 1986.

[18] H. H. Huang and C. T. Sun, "Anomalous wave propagation in a one-dimensional acoustic metamaterial having simultaneously negative mass density and Young's modulus," Journal of Acoustical Society of America, vol. 132, pp. 2887-2895, 2012.

[19] H. H. Huang and C. T. Sun, "Theoretical investigation of the behavior of an acoustic metamaterial with extreme Young's modulus," Journal of Mechanical Physics and Solids, vol. 59, pp. 2070-2081, 2011.

[20] N. Fang, D. Xi, J. Xu, M. Ambati, W. Srituravanich, C. Sun, et al., "Ultrasonic metamaterials with negative modulus," Nature Materials, vol. 5, pp. 452-456, 2006.

[21] S. H. Lee, C. M. Park, Y. M. Seo, Z. G. Wang, and C. K. Kim, "Acoustic metamaterial with negative density," Physics Letters A, vol. 373, pp. 4464-4469, 2009.

[22] S. H. Lee, C. M. Park, Y. M. Seo, Z. G. Wang, and C. K. Kim, "Composite Acoustic Medium with Simultaneously Negative Density and Modulus," Physical Review Letters, vol. 104, p. 054301, 2010.

[23] Z. Liu, C. T. Chan, P. Sheng, A. L. Goertzen, and J. H. Page, "Elastic wave scattering by periodic structures of spherical objects: Theory and experiment," Phys. Rev. B, vol. 62, pp. 2446-2457, 2000. 
[24] P. Sheng, X. X. Zhang, Z. Liu, and C. T. Chan, "Locally resonant sonic materials," Physica B, vol. 338, pp. 201-205, 2003.

[25] J. M. Manimala and C. T. Sun, "Microstructural design studies for locally dissipative acoustic metamaterials," Journal of Applied Physics, vol. 115, p. 023518, 2014.

[26] J. M. Manimala, H. H. Huang, C. T. Sun, R. Snyder, and S. Bland, "Dynamic load mitigation using negative effective mass structures," Engineering Structures, vol. 80, pp. 458-468, 2014.

[27] E. Baravelli, M. Carrara, and M. Ruzzene, "High stiffness, high damping chiral metamaterial assemblies for low-frequency applications," in Health Monitoring of Structural and Biological Systems, San Dieo, USA, 2013, pp. 86952K-86952K10.

[28] H. H. Huang and C. T. Sun, "Wave attenuation mechanism in an acoustic metamaterial with negative effective mass density," New Journal of Physics, vol. 11, p. 013003, 2009.

[29] J. Li and C. T. Chan, "Double-negative acoustic metamaterial," Physical Review E, vol. 70, p. 055602, 2004.

[30] M. Hirsekorn, "Small-size sonic crystals with strong attenuation bands in the audible frequency range," Applied Physics Letters, vol. 84, pp. 3364-3366, 2004.

[31] R. K. Narisetti, M. J. Leamy, and M. Ruzzene, "A perturbation approach for predicting wave propagation in one-dimensional nonlinear periodic structures," Journal of Vibration and Acoustics, vol. 132, p. 031001, 2010. 
[32] B. S. Lazarov and J. S. Jensen, "Low-frequency band gaps in chains with attached non-linear oscillators," International Journal of Non-Linear Mechanics, vol. 42, pp. 1186-1193, 2007.

[33] J. M. Manimala and C. T. Sun, "Numerical Investigation of Amplitude-Dependent Dynamic Response in Acoustic Metamaterials with Nonlinear Oscillators," Journal of Acoustical Society of America special issue on Acoustic Metamaterials, 2016.

[34] Z. Dibin, J. T. Michael, and P. B. Stephen, "Strategies for increasing the operating frequency range of vibration energy harvesters: a review," Measurement Science and Technology, vol. 21, p. 022001, 2010.

[35] R. S. Lakes and W. J. Drugan, "Dramatically stiffer elastic composite materials due to a negative stiffness phase?," Journal of the Mechanics and Physics of Solids, vol. 50, pp. 979-1009, 2002.

[36] L. Kashdan, C. Conner Seepersad, M. Haberman, and P. S. Wilson, "Design, fabrication, and evaluation of negative stiffness elements using SLS," Rapid Prototyping Journal, vol. 18, pp. 194-200, 2012.

[37] Y. Li, J. Lan, B. Li, X. Liu, and J. Zhang, "Nonlinear effects in an acoustic metamaterial with simultaneous negative modulus and density," Journal of Applied Physics, vol. 120, p. 145105, 2016.

[38] B. A. Fulcher, D. W. Shahan, M. R. Haberman, C. Conner Seepersad, and P. S. Wilson, "Analytical and Experimental Investigation of Buckled Beams as Negative Stiffness Elements for Passive Vibration and Shock Isolation Systems," Journal of Vibration and Acoustics, vol. 136, pp. 031009-031009, 2014. 
[39] X. Liu, X. Huang, and H. Hua, "On the characteristics of a quasi-zero stiffness isolator using Euler buckled beam as negative stiffness corrector," Journal of Sound and Vibration, vol. 332, pp. 3359-3376, 2013.

[40] A. H. Nayfeh and D. T. Mook, Nonlinear oscillations: John Wiley \& Sons, 2008.

[41] D. D. Quinn, S. Hubbard, N. Wierschem, M. A. Al-Shudeifat, R. J. Ott, J. Luo, et al., "Equivalent modal damping, stiffening and energy exchanges in multi-degreeof-freedom systems with strongly nonlinear attachments," Proceedings of the Institution of Mechanical Engineers, Part K: Journal of Multi-body Dynamics, vol. 226, pp. 122-146, 2012.

[42] N. Boechler, G. Theocharis, and C. Daraio, "Bifurcation-based acoustic switching and rectification," Nature Materials, vol. 10, pp. 665-668, 2011.

[43] K. Bertoldi and M. C. Boyce, "Mechanically triggered transformations of phononic band gaps in periodic elastomeric structures," Physical Review B, vol. 77, p. 052105, 2008.

[44] K. L. Manktelow, M. J. Leamy, and M. Ruzzene, "Analysis and Experimental Estimation of Nonlinear Dispersion in a Periodic String," Journal of Vibration and Acoustics, vol. 136, p. 031016, 2014.

[45] J. M. Manimala, "Dynamic behavior of acoustic metamaterials and metaconfigured structures with local oscillators," 2014.

[46] N. Nadkarni, A. F. Arrieta, C. Chong, D. M. Kochmann, and C. Daraio, "Unidirectional Transition Waves in Bistable Lattices," Physical Review Letters, vol. 116, p. 244501, 2016. 
[47] M. C. Smith, "Synthesis of mechanical networks: the inerter," IEEE Transactions on Automatic Control, vol. 47, pp. 1648-1662, 2002.

[48] M. Z. Q. Chen, C. Papageorgiou, F. Scheibe, F.-C. Wang, and M. C. Smith, "The missing mechanical circuit element," IEEE Circuits and Systems Magazine, vol. 09, pp. 10-26, 2009.

[49] M. Z. Q. Chen, Y. Hu, L. Huang, and G. Chen, "Influence of inerter on natural frequencies of vibration systems," Journal of Sound and Vibration, vol. 333, pp. 1874-1887, 2014.

[50] I. F. Lazar, S. A. Neild, and D. J. Wagg, "Using an inerter-based device for structural vibration suppression," Earthquake Engineering and Structural Dynamics, vol. 43, pp. 1129-1147, 2014.

[51] F.-C. Wang, M.-K. Liao, B.-H. Liao, W.-J. Su, and H.-A. Chan, "The performance improvements of train suspension systems with mechanical networks employing inerters," Vehicle System Dynamics, vol. 47, pp. 805-830, 2009.

[52] M. C. Smith and F.-C. Wang, "Performance Benefits in Passive Vehicle Suspensions Employing Inerters," Vehicle System Dynamics, vol. 42, pp. 235-257, 2004.

[53] M. Z. Q. Chen, H. Yinlong, and D. Baozhu, "Suspension performance with one damper and one inerter," in Control and Decision Conference (CCDC), 2012 24th Chinese, Taiyuan, China, 2012, pp. 3534-3539.

[54] S. Evangelou, D. J. N. Limebeer, R. S. Sharp, and M. C. Smith, "Mechanical Steering Compensators for High-Performance Motorcycles," Journal of Applied Mechanics, vol. 74, pp. 332-346, 2006. 
[55] Y. Hu, M. Z. Q. Chen, and Z. Shu, "Passive vehicle suspensions employing inerters with multiple performance requirements," Journal of Sound and Vibration, vol. 333, pp. 2212-2225, 2014.

[56] M. Z. Q. Chen, Y. Hu, C. Li, and G. Chen, "Performance Benefits of Using Inerter in Semiactive Suspensions," IEEE Transactions on Control Systems Technology, vol. 23, pp. 1571-1577, 2015.

[57] Y. Hu, M. Z. Q. Chen, Z. Shu, and L. Huang, "Analysis and optimisation for inerter-based isolators via fixed-point theory and algebraic solution," Journal of Sound and Vibration, vol. 346, pp. 17-36, 2015.

[58] C. Papageorgiou and M. C. Smith, "Laboratory experimental testing of inerters," in Decision and Control, 2005 and 2005 European Control Conference. CDCECC '05. 44th IEEE Conference on, Seville, Spain, 2005, pp. 3351-3356.

[59] L. Marian and A. Giaralis, "Optimal design of a novel tuned mass-damper-inerter (TMDI) passive vibration control configuration for stochastically support-excited structural systems," Probabilistic Engineering Mechanics, vol. 38, pp. 156-164, 2014.

[60] Y. Hu and M. Z. Q. Chen, "Performance evaluation for inerter-based dynamic vibration absorbers," International Journal of Mechanical Sciences, vol. 99, pp. 297-307, 2015.

[61] O. V. Rudenko, "Giant nonlinearities in structurally inhomogeneous media and the fundamentals of nonlinear acoustic diagnostic techniques," Physics-Uspekhi, vol. 49, pp. 69-87, 2006. 
[62] X. Guo, Z. Lin, J. Tu, B. Liang, J. Cheng, and D. Zhang, "Modeling and optimization of an acoustic diode based on micro-bubble nonlinearity," The Journal of the Acoustical Society of America, vol. 133, pp. 1119-1125, 2013.

[63] B. P. Mann and N. D. Sims, "Energy harvesting from the nonlinear oscillations of magnetic levitation," Journal of Sound and Vibration, vol. 319, pp. 515-530, 2009.

[64] C. V. Jutte and S. Kota, "Design of Nonlinear Springs for Prescribed LoadDisplacement Functions," Journal of Mechanical Design, vol. 130, pp. 081403081403, 2008.

[65] L. E. Kinsler, A. R. Frey, A. B. Coppens, and J. V. Sanders, "Fundamentals of acoustics," Fundamentals of Acoustics, 4th Edition, by Lawrence E. Kinsler, Austin R. Frey, Alan B. Coppens, James V. Sanders, pp. 560. ISBN 0-471-847895. Wiley-VCH, December 1999., p. 560, 1999.

[66] D. Spreemann, B. Folkmer, and Y. Manoli, "Realization of nonlinear hardening springs with predefined characteristic for vibration transducers based on beam structures," MikroSystemTechnik, 2011.

[67] G. Theocharis, N. Boechler, and C. Daraio, "Nonlinear periodic phononic structures and granular crystals," Acoustic Metamaterials and Phononic Crystals. Springer Berlin Heidelberg, 2013. 217-251. 


\section{APPENDIX-I \\ MATLAB CODE TO COMPUTE THE CANTILEVER ROOT PROFILE FOR PRE-DEFINED NONLINEARITY}

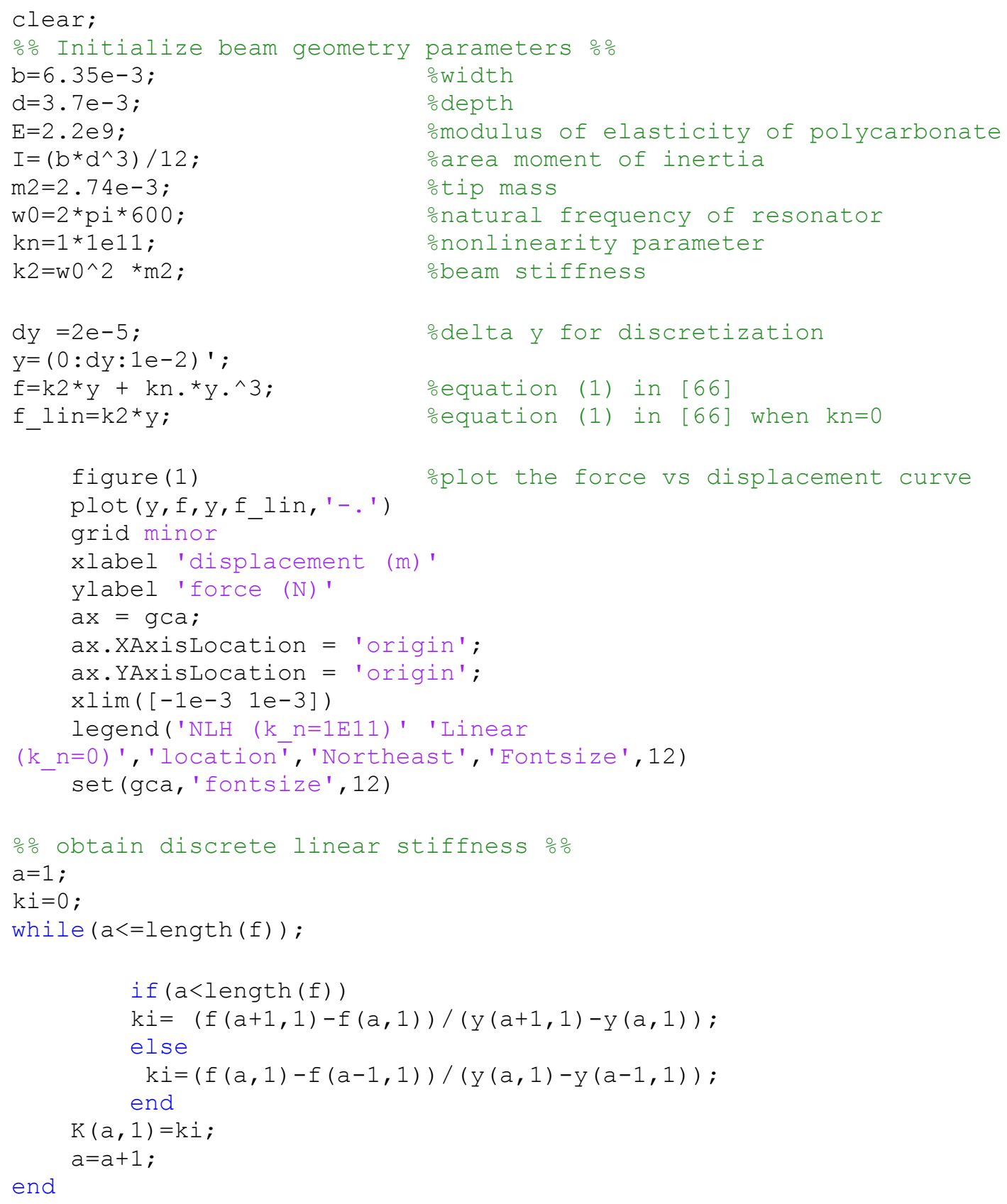




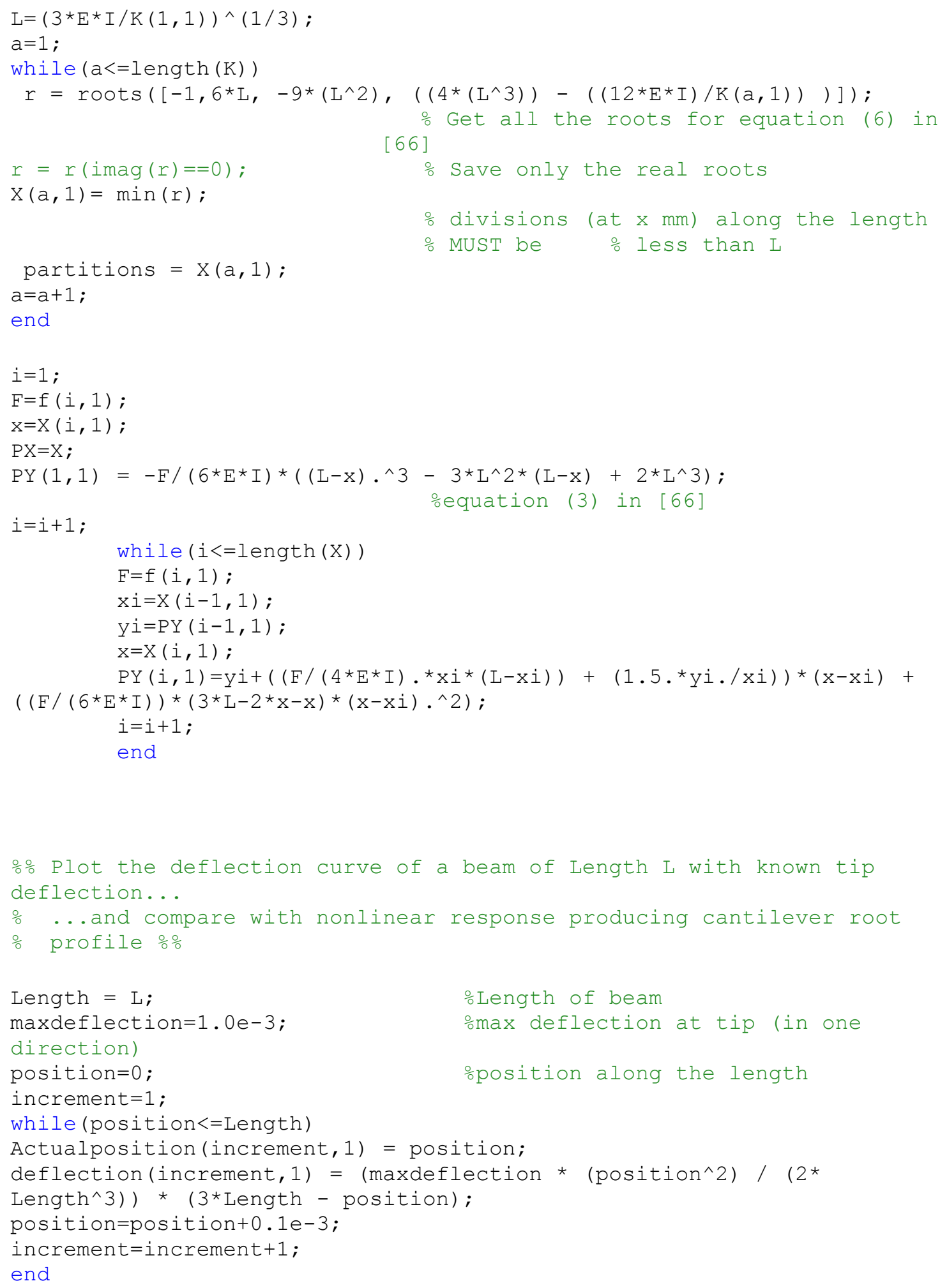


\% Plot the beam deflection curve against root profile figure (2)

plot (1000*PY, $1000 * \mathrm{PX}$, ' - ' , 1000*deflection, $1000 *$ Actualposition, ' -. ' ) ; grid off

$\operatorname{axis}\left(\left[\begin{array}{llll}0 & 3.5 & 0 & 20 e 0\end{array}\right)\right.$

xlabel ('position from root (mm)','Fontsize',12);

ylabel('position along length (mm)','Fontsize',12);

legend('root profile' 'deflection

curve',' location', 'southeast', 'Fontsize', 12)

set (gca, 'fontsize', 12) 
VITA

Prateek P. Kulkarni

Candidate for the Degree of

Master of Science

\title{
Thesis: NONLINEAR AND INERTANT ACOUSTIC METAMATERIALS AND THEIR DEVICE IMPLICATIONS
}

\author{
Major Field: Mechanical and Aerospace Engineering \\ Biographical:
}

Education:

Completed the requirements for the Master of Science in Mechanical and Aerospace Engineering at Oklahoma State University, Stillwater, Oklahoma in December 2016.

Completed the requirements for the Bachelor of Engineering in Mechanical and Engineering at Birla Institute of Technology in May 2012.

\section{Experience:}

Graduate Research Assistant at Solid and Structural Dynamics Lab, School of Mechanical and Aerospace Engineering, Oklahoma State University, Stillwater, Ok.

Graduate Teaching Assistant, Elementary Dynamics (ENSC 2123), at School of Mechanical and Aerospace Engineering, Oklahoma State University, Stillwater, Ok.

Professional Memberships:

-American Society of Mechanical Engineers 